\title{
Galerkin Boundary Element Methods for Electromagnetic Scattering
}

\author{
A. Buffa ${ }^{1}$ and R. Hiptmair ${ }^{2}$ \\ 1 Istituto di Matematica applicate e tecnologie informatiche del CNR, Pavia, Italy, \\ annalisa@ian.pv.cnr.it \\ 2 Seminar für Angewandte Mathematik, ETH Zürich, CH-8092 Zürich, \\ hiptmair@sam.math.ethz.ch
}

in Computational Methods in Wave Propagation, M. Ainsworth, ed., Springer, New York, 2003, pp. 85-126. In print

Extended version with new appendix on Scattering from coated dielectric objects. Draft version December 12, 2007.

Summary. Methods based on boundary integral equations are widely used in the numerical simulation of electromagnetic scattering in the frequency domain. This article examines a particular class of these methods, namely the Galerkin boundary element approach, from a theoretical point of view. Emphasis is put on the fundamental differences between acoustic and electromagnetic scattering. The derivation of various boundary integral equations is presented, properties of their discretized counterparts are discussed, and a-priori convergence estimates for the boundary element solutions are rigorously established.

Key words: Electromagnetic scattering, boundary integral equations, boundary element methods

\section{Introduction}

The numerical simulation of electromagnetic scattering aims at computing the interaction of electromagnetic waves with a physical body, the so-called scatterer. The scatterer occupies a bounded domain $\Omega_{s}$ in three-dimensional affine space $\mathbb{R}^{3}$. In general, $\Omega_{s}$ will have Lipschitz-continuous boundary $\Gamma:=\partial \Omega_{s}$ [41, Section 1.2], which can be equipped with an exterior unit normal vectorfield $\mathbf{n} \in \boldsymbol{L}^{\infty}(\Gamma)$. With boundary element methods in mind, we do not lose generality by considering only piecewise smooth $\Omega_{s}$, i.e., curvilinear Lipschitz polyhedra in the parlance of [35].

We only consider linear materials and time-harmonic electromagnetic fields of angular frequency $\omega>0$. Excitation is provided by the fields $\mathbf{e}_{i}, \mathbf{h}_{i}$ of an incident (plane) wave. Under these circumstances we can derive the following transmission problem from Maxwell's equations [31, Ch. 6]: 


$$
\begin{gathered}
\operatorname{curl} \mathbf{e}=-i \omega \underline{\boldsymbol{\mu}} \mathbf{h}, \quad \operatorname{curl} \mathbf{h}=i \omega \underline{\mathbf{\epsilon}} \mathbf{e} \quad \text { in } \Omega_{s} \cup \Omega^{\prime}, \\
\gamma_{\mathbf{t}}^{+} \mathbf{e}-\gamma_{\mathbf{t}}^{-} \mathbf{e}=-\gamma_{\mathbf{t}}^{+} \mathbf{e}_{i}, \quad \gamma_{\mathbf{t}}^{+} \mathbf{h}-\gamma_{\mathbf{t}}^{-} \mathbf{h}=-\gamma_{\mathbf{t}}^{+} \mathbf{h}_{i} \quad \text { on } \Gamma, \\
\int_{\partial B_{r}}\left|\gamma_{\mathbf{t}} \mathbf{h} \times \mathbf{n}+\gamma \mathbf{e}\right|^{2} \mathrm{~d} S \rightarrow 0 \quad \text { for } r \rightarrow \infty .
\end{gathered}
$$

Here and below, $B_{r}$ denotes a ball of radius $r$ centered in the origin, $\gamma_{\mathbf{t}} \mathbf{u}$ stands for the tangential trace $\mathbf{u} \times \mathbf{n}$, and superscripts - and + tag traces onto $\Gamma$ from $\Omega_{s}$ and $\Omega^{\prime}:=\mathbb{R}^{3} \backslash \bar{\Omega}$, respectively. The vectorfields $\mathbf{e}=\mathbf{e}(\mathbf{x}), \mathbf{h}=\mathbf{h}(\mathbf{x})$ represent the unknown complex amplitudes (phasors) of the electric and magnetic field, respectively. The material parameters $\underline{\mu}=\underline{\mu}(\mathbf{x})$ (permeability tensor), $\underline{\epsilon}=\underline{\epsilon}(\mathbf{x})$ (dielectric tensor), $\mathbf{x} \in \mathbb{R}^{3}$, are uniformly positive definite and bounded. In fact, information on the scatterer is completely contained in $\underline{\mu}$ and $\underline{\epsilon}$ : inside $\Omega_{s}$ they may vary, but in the "air region" $\Omega^{\prime}$ both material parameters agree with the constants $\mu_{0}>0$ and $\epsilon_{0}$, respectively. At $\infty$ the so-called Silver-Müller radiation conditions are imposed.

This system of equations can always be reduced to a second order wave equation in terms either of the electric or the magnetic field, e.g., e satisfies the electric wave equation

$$
\operatorname{curl} \underline{\boldsymbol{\mu}}^{-1} \operatorname{curl} \mathbf{e}-\omega^{2} \underline{\epsilon} \mathbf{e}=0 \quad \text { in } \Omega_{s} \cup \Omega^{\prime} .
$$

Note that the uniqueness of solutions of the system (1) is a direct consequence of Rellich's Lemma [24,53].

Apart from generic dielectric and even lossy scatterers the following special situations are of practical interest.

- The scatterer is assumed to be a "perfect conductor" in which no electric field can exist. This leads to an exterior Dirichlet problem for the electric wave equations in $\Omega^{\prime}$, because the transmission conditions in (1) are replaced by the boundary condition $\gamma_{\mathbf{t}}^{+} \mathbf{e}=-\gamma_{\mathbf{t}}^{+} \mathbf{e}_{i}$ on $\Gamma$ for the electric field.

- If the scatterer is a thin perfectly conducting sheet, we arrive at a screen problem. In this case $\Omega_{s}=\emptyset$ and $\Gamma$ becomes a compact piecewise smooth two-dimensional surface with boundary. As before, we demand $\gamma_{\mathbf{t}} \mathbf{e}=-\gamma_{\mathbf{t}} \mathbf{e}_{i}$ on both sides of $\Gamma$. For screen problems $\Omega^{\prime}$ does not possess a Lipschitz boundary any more. Moreover, the screen $\Gamma$ itself might not even be a Lipschitz surface itself, in case it branches. The resulting mathematical complications are treated in [16].

- If the scatterer is a good conductor with smooth surface, its impact on the fields can be modelled by impedance boundary conditions (Leontovich boundary conditions) $[3,10,54]$

$$
\gamma_{\mathbf{t}}^{+} \mathbf{e}-\underline{\boldsymbol{\eta}}\left(\gamma_{\mathbf{t}}^{+} \mathbf{h} \times \mathbf{n}\right)=\underline{\boldsymbol{\eta}}\left(\gamma_{\mathbf{t}}^{+} \mathbf{h}_{i} \times \mathbf{n}\right)-\gamma_{\mathbf{t}}^{+} \mathbf{e}_{i} \quad \text { on } \Gamma .
$$

The surface impedance $\underline{\boldsymbol{\eta}}$ is a complex tensor with uniformly positive definite real part and non-zero imaginary part. 
All these problems have in common that scattered fields on the unbounded domain $\Omega^{\prime}$ have to be determined. As the material coefficients are constant in $\Omega^{\prime}$, boundary integral equation methods are perfectly suited for this job. In addition, they are posed on the two-dimensional surface $\Gamma$, which relieves us from meshing (a part of) $\Omega^{\prime}$. In the case of complicated geometries this is a strong point in favour of boundary integral equation methods, compared to volume based schemes with absorbing boundary conditions ( $c f$. the contribution of T. Hagstrom on absorbing layers and radiation boundary conditions in this collection) at an artificial cut-off boundary.

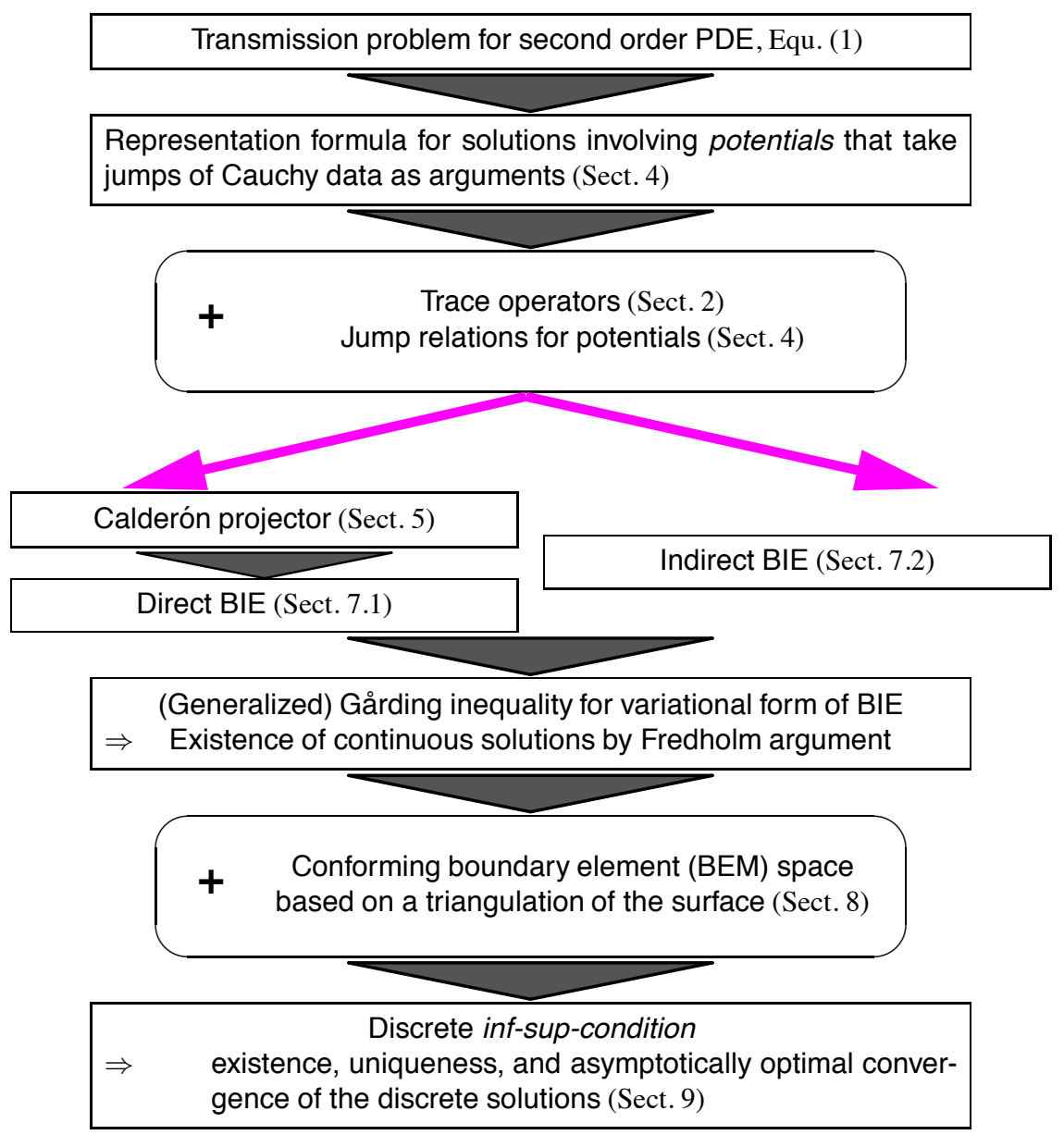

Fig. 1. "Road map" for the derivation and analysis of Galerkin boundary element methods for electromagnetic scattering $(\mathrm{BIE}=$ boundary integral equation) 
In this article we will exclusively deal with the Galerkin method for the discretization of the boundary integral equations. It is based on variational formulations in suitable trace spaces. This permits us to use powerful tools from functional analysis. They pave the way for a rigorous and comprehensive convergence theory. We acknowledge that several other numerical methods based on boundary integral formulations exist and are widely used alternatives to Galerkin schemes:

- the collocation method, which can be regarded as a special Petrov-Galerkin approach [42, Sect. 4.4]

- the method of source potentials, which requires a second surface away from $\Gamma$, on which a source distribution is sought. An example of this method for electromagnetic scattering is analysed in [43].

- Nyström methods, which directly tackle the boundary integral equations by means of a quadrature rule. For an exposition we refer to the contribution of $\mathrm{O}$. Bruno in this volume and to [49, Ch. 12].

Unfortunately the theoretical understanding of these methods is rudimentary in comparison with Galerkin schemes. For this reason we us restrict the presentation to Galerkin methods.

As far as Galerkin boundary element methods are concerned, there is a fairly canonical approach to their construction and theoretical examination. This standard procedure is depicted in the flowchart of Fig. 1. The plan of this paper closely follows these lines.

We point out that issues of implementation and efficient solution of the resulting linear systems of equations are not covered by this article. We will also skip quite a few proofs, which the reader may look up in the research papers that underly this survey. In particular, we mention $[15,20]$ as main references for Sect. 2.1, [45, Ch. 5] for Sect. 3, [31, Ch. 6] and [16,21] as regards Sects. 4-9, and [44] as source for Sect. 10.

\section{Function Spaces and Traces}

In order to write problem (1) or equivalent formulations of it in a mathematically rigorous way, we need a precise characterization of the function spaces, on which the equations are posed. This section is devoted to definitions and main properties of function spaces which are concerned with the rigorous formulation of the problem (1). The first section concerns spaces on the domain, either $\Omega_{s}, \Omega^{\prime}=\mathbb{R}^{3} \backslash \overline{\Omega_{s}}$ or $\mathbb{R}^{3}$, while in the second we define and characterize suitable spaces on the manifold $\Gamma$, which will be of key importance for the definition of integral operators.

\subsection{Function spaces in the domain}

Let $\Omega \subseteq \mathbb{R}^{3}$ be any of the sets $\Omega_{s}, \Omega^{\prime}, \mathbb{R}^{3}$ and define the Fréchet space $\boldsymbol{L}_{\text {loc }}^{2}(\Omega)$ of complex, vector valued, locally square integrable functions $\mathbf{u}: \Omega \rightarrow \mathbb{C}^{3}$. We also 
make use of the Sobolev spaces $\boldsymbol{H}_{\text {loc }}^{s}(\Omega), s \geq 0$ with the convention $\boldsymbol{H}^{0} \equiv \boldsymbol{L}^{2}$ (see, e.g., [1] for definitions). The sub-fix loc is systematically removed when $\Omega$ is bounded: in this case, the $\boldsymbol{H}^{s}(\Omega)$ are Hilbert spaces endowed with the natural graph-norm $\|\mathbf{u}\|_{\boldsymbol{H}^{s}\left(\Omega_{s}\right)}$ and semi-norm $|\mathbf{u}|_{\boldsymbol{H}^{s}\left(\Omega_{s}\right)}$, respectively [1]. Round brackets will consistently be used to express inner products.

With $\mathbf{d}$ a first order differential operator, we define for any $s \geq 0$

$$
\begin{gathered}
\boldsymbol{H}_{\mathrm{loc}}^{s}(\mathbf{d}, \Omega):=\left\{\mathbf{u} \in \boldsymbol{H}_{\mathrm{loc}}^{s}(\Omega): \mathbf{d u} \in \boldsymbol{H}_{\mathrm{loc}}^{s}(\Omega)\right\} \\
\boldsymbol{H}_{\mathrm{loc}}^{s}(\mathbf{d} 0, \Omega):=\left\{\mathbf{u} \in \boldsymbol{H}_{\mathrm{loc}}^{s}(\Omega): \mathbf{d u}=0\right\}
\end{gathered}
$$

When $s=0$, we simplify the notation by setting $\boldsymbol{H}^{0}=\boldsymbol{H}$. If $\Omega$ is bounded, $\boldsymbol{H}_{\mathrm{loc}}^{s}(\mathbf{d}, \Omega)$ is endowed with the graph norm $\|\cdot\|_{\boldsymbol{H}^{s}(\mathbf{d}, \Omega)}^{2}:=\|\cdot\|_{\boldsymbol{H}^{s}(\Omega)}^{2}+\|\mathbf{d} \cdot\|_{\boldsymbol{H}^{s}(\Omega)}^{2}$ and seminorm $|\cdot|_{\boldsymbol{H}^{s}(\mathbf{d}, \Omega)}^{2}:=|\cdot|_{\boldsymbol{H}^{s}(\Omega)}^{2}+|\mathbf{d} \cdot|_{\boldsymbol{H}^{s}(\Omega)}^{2}$. This defines the spaces $\boldsymbol{H}^{s}(\operatorname{curl}, \Omega), \boldsymbol{H}^{s}(\operatorname{div}, \Omega)$ and $\boldsymbol{H}^{s}(\operatorname{curl} 0, \Omega), \boldsymbol{H}^{s}(\operatorname{div} 0, \Omega)$.

¿From Gauß' theorem we obtain integration by parts formulae for the spaces $\boldsymbol{H}\left(\operatorname{curl}, \Omega_{s}\right), \boldsymbol{H}\left(\operatorname{div}, \Omega_{s}\right)$. If $\mathbf{u}, \mathbf{v} \in C^{\infty}\left(\overline{\Omega_{s}}\right)^{3}$ and $p \in C^{\infty}\left(\overline{\Omega_{s}}\right)$, then we have $\operatorname{div}(\mathbf{u} p)=\operatorname{div} \mathbf{u} p+\mathbf{u} \cdot \nabla p$ and $\operatorname{div}(\mathbf{u} \times \mathbf{v})=\operatorname{curl} \mathbf{u} \cdot \mathbf{v}-\operatorname{curl} \mathbf{v} \cdot \mathbf{u}$, and, finally, $(\mathbf{u} \times \mathbf{v}) \cdot \mathbf{n}=-(\mathbf{u} \times \mathbf{n}) \cdot \mathbf{v}$ on the boundary $\Gamma$. These imply the following formulae:

$$
\begin{aligned}
& \int_{\Omega_{s}} \operatorname{div}(\mathbf{u} p)=\int_{\Omega_{s}}(\operatorname{div} \mathbf{u} p+\mathbf{u} \cdot \nabla p) \mathrm{d} \mathbf{x}=\int_{\Gamma} p \mathbf{u} \cdot \mathbf{n} \mathrm{d} S, \\
& \int_{\Omega_{s}}(\mathbf{u} \cdot \operatorname{curl} \mathbf{v}-\operatorname{curl} \mathbf{u} \cdot \mathbf{v}) \mathrm{d} \mathbf{x}=\int_{\Gamma}(\mathbf{u} \times \mathbf{n}) \cdot \mathbf{v}_{\mid \Gamma} \mathrm{d} S .
\end{aligned}
$$

These formulae suggest the definitions of the mappings $\gamma_{\mathbf{t}}: \mathbf{u} \mapsto \mathbf{u}_{\mid \Gamma} \times \mathbf{n}$ and $\gamma_{\mathbf{n}}: \mathbf{u} \mapsto \mathbf{u}_{\mid \Gamma} \cdot \mathbf{n}, \mathbf{u} \in C^{\infty}\left(\overline{\Omega_{s}}\right)^{3}$.

The trace theorem for $\boldsymbol{H}^{1}(\Omega)$ [40, Theorem 1.5.1.1] shows that the tangential trace $\gamma_{\mathbf{t}}: \boldsymbol{C}^{\infty}(\bar{\Omega}) \mapsto \boldsymbol{L}^{\infty}(\Gamma)$ and the normal trace: $\gamma_{\mathbf{n}}: \boldsymbol{C}^{\infty}(\bar{\Omega}) \mapsto L^{\infty}(\Gamma)$ are continuous as mappings $\boldsymbol{H}(\operatorname{curl} ; \Omega) \mapsto \boldsymbol{H}^{-\frac{1}{2}}(\Gamma)$ and $\boldsymbol{H}(\operatorname{div} ; \Omega) \mapsto H^{-\frac{1}{2}}(\Gamma)$, respectively. Here, $H^{-\frac{1}{2}}(\Gamma)$ and $\boldsymbol{H}^{-\frac{1}{2}}(\Gamma)$ are the dual space of $H^{\frac{1}{2}}(\Gamma)$ and $\boldsymbol{H}^{\frac{1}{2}}(\Gamma):=\left(H^{\frac{1}{2}}(\Gamma)\right)^{3}$, respectively, with respect to the pivot spaces $L^{2}(\Gamma) / \boldsymbol{L}^{2}(\Gamma)$. Consequently, the traces can be extended to $\boldsymbol{H}(\operatorname{curl} ; \Omega)$ and $\boldsymbol{H}(\operatorname{div} ; \Omega)$, respectively.

In the sequel we will consider the electric wave equation (2). Now, since the fact that the field is a locally square-integrable function satisfying curl curl $\mathbf{u}-\mathbf{u}=0$, we can conclude that curl curl $\mathbf{u}$ is locally square-integrable, too. Hence, the space

$$
\boldsymbol{H}_{\mathrm{loc}}\left(\operatorname{curl}^{2}, \Omega\right):=\left\{\mathbf{u} \in \boldsymbol{H}_{\mathrm{loc}}(\operatorname{curl} ; \Omega), \operatorname{curl} \operatorname{curl} \mathbf{u} \in \boldsymbol{L}_{\mathrm{loc}}^{2}(\Omega)\right\}
$$

comes into play as the natural space for the solutions of the electric/magnetic wave equation with constant coefficients. It will be crucial for meaningful strong formulations of electromagnetic transmission problems. 


\subsection{Function spaces on the manifold $\Gamma$}

Recall that through local charts one defines standard Sobolev spaces on the manifold $\Gamma=\partial \Omega_{s}$. We denote them as $H^{s}(\Gamma), \mathbf{H}^{s}(\Gamma), s \in[-1,1]$, for scalars and vectors, respectively. We saw that the tangential trace operator $\gamma_{\mathbf{t}}$ possesses an interpretation as a continuous mapping $\boldsymbol{H}(\mathbf{c u r l} ; \Omega) \mapsto \boldsymbol{H}^{-\frac{1}{2}}(\Gamma)$. This is actually sufficient for the understanding of homogeneous boundary conditions for fields in the Hilbert space context. However, in order to impose meaningful non-homogeneous boundary conditions or, even more important, to lay the foundations for boundary integral equations we need to identify a proper trace space " $X(\Gamma)$ " of $\boldsymbol{H}(\operatorname{curl} ; \Omega), \Omega \subset \mathbb{R}^{3}$ a "generic" domain. It has to meet two essential requirements:

1. The inner product on $X(\Gamma)$ has an intrinsic definition that does not rely on the embedding of $\Gamma$ into $\mathbb{R}^{3}$, i.e, $X(\Gamma)$ should have an interpretation as sections of the tangent bundle to $T \Gamma$ of $\Gamma$.

2. We demand that $\gamma_{\mathbf{t}}: \boldsymbol{H}(\mathbf{c u r l} ; \Omega) \mapsto X(\Gamma)$ is continuous and surjective.

Note that the same issue for the operator $\gamma_{\mathbf{n}}: \boldsymbol{H}(\operatorname{div} ; \Omega) \rightarrow H^{-\frac{1}{2}}(\Gamma)$ was resolved a long time ago [39, Sect. I.2.2].

We emphasize that for the discussion of traces it hardly matters, whether $\Omega$ is bounded or not. We assume in this section that $\Omega$ is bounded (this allows for integration on $\Omega$ ), but with this slight change, the results of this section remain true also for unbounded domains, in particular, the open complement of $\Omega$.

Smooth boundaries. To illustrate ideas, we first consider a $C^{\infty}$-smooth $\Gamma$. Then the Sobolev spaces $\boldsymbol{H}^{s}(\Gamma)$ of functions and $\boldsymbol{H}_{\mathbf{t}}^{s}(\Gamma)$ of tangential vector-fields, as well as differential surface operators (we shall use the self evident notation $\left.\operatorname{div}_{\Gamma}, \operatorname{curl}_{\Gamma}, \operatorname{curl}_{\Gamma}, \ldots\right)$ can be defined for all $s \in \mathbb{R}$ using local charts and transformations [24, Sect. 3.1, Appendix] [53, Sect. 2.5.2]. It is a classical result that smooth functions on $\Gamma$ are dense in all these spaces. Standard trace and tangential trace generate continuous and surjective operators $\gamma: H^{s+\frac{1}{2}}(\Omega) \mapsto H^{s}(\Gamma)$ and $\gamma_{\mathbf{t}}: \boldsymbol{H}^{s+\frac{1}{2}}(\Omega) \mapsto \boldsymbol{H}_{\mathbf{t}}^{s}(\Gamma)$ for all $s>0$, where

$$
\boldsymbol{H}_{\mathbf{t}}^{s}(\Gamma) \cong\left\{\boldsymbol{\phi} \in \boldsymbol{H}^{s}(\Gamma), \boldsymbol{\phi} \cdot \mathbf{n}=0\right\} \subset \boldsymbol{L}_{\mathbf{t}}^{2}(\Gamma)
$$

are Sobolev spaces of tangential vector-fields. We denote by $\boldsymbol{H}_{\mathbf{t}}^{-s}(\Gamma)$ the dual space of $\boldsymbol{H}_{\mathbf{t}}^{s}(\Gamma)$ with $\boldsymbol{L}_{\mathbf{t}}^{2}(\Gamma)$ as a pivot space. Angle brackets will designate the duality pairings. Now, since for any $\mathbf{u} \in \boldsymbol{H}(\operatorname{curl} ; \Omega)$ we have $\gamma_{\mathbf{t}} \mathbf{u} \cdot \mathbf{n}=0$, thus $\gamma_{\mathbf{t}} \mathbf{u} \in$ $\boldsymbol{H}_{\mathbf{t}}^{-1 / 2}(\Gamma)$. Moreover, using both (5) and (6), we can easily see that

$$
\operatorname{div}_{\Gamma}\left(\gamma_{\mathbf{t}} \mathbf{u}\right)=\gamma_{\mathbf{n}}(\operatorname{curl} \mathbf{u}) \quad \forall \mathbf{u} \in \boldsymbol{H}(\operatorname{curl} ; \Omega),
$$

which implies $\operatorname{div}_{\Gamma}\left(\gamma_{\mathbf{t}} \mathbf{u}\right) \in H^{-1 / 2}(\Gamma)$.

Now, it is natural to define the space

$$
\boldsymbol{T} \boldsymbol{H}^{s}\left(\operatorname{div}_{\Gamma} ; \Gamma\right):=\left\{\boldsymbol{\mu} \in \boldsymbol{H}_{\mathbf{t}}^{s}(\Gamma), \operatorname{div}_{\Gamma} \boldsymbol{\mu} \in H^{s}(\Gamma)\right\} .
$$


The tangential trace $\gamma_{\mathbf{t}}: \boldsymbol{H}(\operatorname{curl} ; \Omega) \mapsto \boldsymbol{T} \boldsymbol{H}^{-\frac{1}{2}}\left(\operatorname{div}_{\Gamma} ; \Gamma\right)$ turns out to be continuous and surjective [53, Theorem 5.4.2]. For smooth surfaces the issue of tangential traces in $\boldsymbol{H}(\mathbf{c u r l} ; \Omega)$ was investigated in the papers of L. Paquet [55] and Alonso/Valli [2]. A survey of the results is also given in the monographs by M. Cessenat [24, Sect. 2.1] and J.-C. Nédélec [53, Sect. 5.4.1].

Moreover, if we define the anti-symmetric pairing

$$
\langle\boldsymbol{\mu}, \boldsymbol{\eta}\rangle_{\boldsymbol{\tau}, \Gamma}:=\int_{\Gamma}(\boldsymbol{\mu} \times \mathbf{n}) \cdot \boldsymbol{\eta} \mathrm{d} S, \quad \boldsymbol{\mu}, \boldsymbol{\eta} \in \boldsymbol{L}_{\mathbf{t}}^{2}(\Gamma),
$$

then we can rewrite (6) as

$$
\int_{\Omega}(\operatorname{curl} \mathbf{u} \cdot \mathbf{v}-\mathbf{u} \cdot \operatorname{curl} \mathbf{v}) \mathrm{d} \mathbf{x}=\left\langle\gamma_{\mathbf{t}} \mathbf{v}, \gamma_{\mathbf{t}} \mathbf{u}\right\rangle_{\boldsymbol{\tau}, \Gamma}
$$

which suggests that the space $\boldsymbol{T} \boldsymbol{H}^{-\frac{1}{2}}\left(\operatorname{div}_{\Gamma} ; \Gamma\right)$ coincides with its dual when using $\langle\cdot, \cdot\rangle_{\boldsymbol{\tau}, \Gamma}$ as duality pairing. This statement will be clarified in the case of non-smooth surfaces at the end of this section.

Piecewise smooth and Lipschitz boundaries. Only recently results have been obtained for non-smooth boundaries. We owe it to the pioneering work of one of the authors together with P. Ciarlet jr., who first examined piecewise smooth boundaries in $[14,17,18]$. The issue of traces of $\boldsymbol{H}(\operatorname{curl} ; \Omega)$ for general Lipschitz-domains was finally settled jointly by one of the authors, M. Costabel and D. Sheen in [20]. These articles and Sect. 2 of [19] supply the main references for the current section.

The challenges faced in the case of piecewise smooth boundaries are highlighted by simple consideration: even if $\mathbf{u} \in \boldsymbol{C}^{\infty}(\bar{\Omega})$ we do not have $\gamma_{\mathbf{t}} \mathbf{u} \in \boldsymbol{H}^{\frac{1}{2}}(\Gamma)$, because the tangential trace is inevitably discontinuous across edges of $\Gamma$. The first consequence of this fact is that $\gamma_{\mathbf{t}} \boldsymbol{H}^{1}(\Omega) \times \mathbf{n} \nsubseteq \gamma_{\mathbf{t}} \boldsymbol{H}^{1}(\Omega)$, although the two objects are both good candidates to be "tangential" vector fields of "regularity" $\frac{1}{2}$. Thus we have to resort to the following definition:

Definition 1. We introduce the Hilbert space $\boldsymbol{H}_{\times}^{s}(\Gamma):=\gamma_{\mathbf{t}}\left(\boldsymbol{H}^{s+1 / 2}(\Omega)\right), s \in$ $(0,1)$, equipped with an inner product that renders $\gamma_{\mathbf{t}}: \boldsymbol{H}^{s+1 / 2}(\Omega) \mapsto \boldsymbol{H}_{\times}^{s}(\Gamma)$ continuous and surjective. Its dual space with respect to the pairing $\langle\cdot, \cdot\rangle_{\boldsymbol{\tau}, \Gamma}$ is denoted by $\boldsymbol{H}_{\times}^{-s}(\Gamma)$.

The dual space is well defined due to the density of $\boldsymbol{H}_{\times}^{\frac{1}{2}}(\Gamma) \subset \boldsymbol{L}_{\mathbf{t}}^{2}(\Gamma)$. The case of smooth and non-smooth surfaces differ considerably, which we aim to highlight by different notations: $\boldsymbol{H}_{\mathbf{t}}^{s}(\Gamma)$ for smooth $\Gamma$ and $\boldsymbol{H}_{\times}^{s}(\Gamma)$ for non-smooth $\Gamma$.

For curvilinear polyhedra this space can be given a more concrete meaning. To that end, write $\Gamma^{1}, \ldots, \Gamma^{P}, P \in \mathbb{N}$, for the finitely many curved polygonal faces of $\Gamma$, i.e. $\Gamma:=\bigcup_{j=1}^{P} \bar{\Gamma}^{j}$, meeting at non-degenerate edges. For any tangential vector $\boldsymbol{\mu}$, we denote by $\boldsymbol{\mu}^{j}$ the restriction of $\boldsymbol{\mu}$ to $\Gamma^{j}$. Then, according to [17, Proposition 1.6] an equivalent norm on $\boldsymbol{H}_{\times}^{\frac{1}{2}}(\Gamma)$ can be expressed as 
$\|\boldsymbol{\mu}\|_{\boldsymbol{H}_{\times}^{\frac{1}{2}}(\Gamma)}^{2}:=\sum_{j=1}^{P}\left\|\boldsymbol{\mu}^{j}\right\|_{\boldsymbol{H}_{\mathbf{t}}^{\frac{1}{2}}\left(\Gamma^{j}\right)}^{2}+\sum_{j=1}^{P} \sum_{i \in \mathcal{I}_{j}{ }_{\Gamma^{j} \times \Gamma^{i}}} \int_{\left|\boldsymbol{\mu}^{i} \cdot \boldsymbol{\nu}^{i j}(\mathbf{x})-\boldsymbol{\mu}^{j} \cdot \boldsymbol{\nu}^{j i}(\mathbf{y})\right|^{2}}^{|\mathbf{x}-\mathbf{y}|^{3}} \mathrm{~d} S^{2}$.

where $\mathcal{I}_{j}$ is the set of indices of smooth components abutting $\Gamma^{j}$, and $\nu^{i j}$ denotes the tangential outer normal to $\Gamma^{i}$ restricted to the edge $\overline{\Gamma^{j}} \cap \overline{\Gamma^{i}}$. Loosely speaking, $\boldsymbol{H}_{\times}^{\frac{1}{2}}(\Gamma)$ contains vector-fields that are in $\boldsymbol{H}_{\mathbf{t}}^{\frac{1}{2}}\left(\Gamma^{j}\right)$ for each face $\Gamma^{j}$ and feature a "weak normal continuity" enforced by the second term in the definition of the norm.

Using (6) and the same reasoning as for regular surfaces, we have that $\gamma_{\mathbf{t}}$ : $\boldsymbol{H}(\operatorname{curl} ; \Omega) \rightarrow \boldsymbol{H}_{\times}^{-\frac{1}{2}}(\Gamma)$ is linear and continuous. In view of (8), we also know that this operator does not admit a right inverse. In order to repeat the argument sketched above for regular domains, we need a theory of differential operators on non-smooth manifolds. We do not want to delve into the details of these developments, and we refer the reader to $[14,16,20]$ for a discussion on the subject. We need only the following definition: for $\mathbf{u} \in C^{\infty}(\bar{\Omega})$ set

$$
\operatorname{div}_{\Gamma} \gamma_{\mathbf{t}} \mathbf{u}:= \begin{cases}\operatorname{div}_{j}\left(\gamma_{\mathbf{t}} \mathbf{u}\right)^{j} & \text { on } \Gamma^{j} \\ \left(\left(\gamma_{\mathbf{t}} \mathbf{u}\right)^{j} \cdot \boldsymbol{\nu}^{i j}+\left(\gamma_{\mathbf{t}} \mathbf{u}\right)^{i} \cdot \boldsymbol{\nu}^{j i}\right) \delta_{i j} & \text { on } \overline{\Gamma^{j}} \cap \overline{\Gamma^{i}}\end{cases}
$$

where $\delta_{i j}$ is the delta distribution (in local coordinates) whose support is the edge $\overline{\Gamma^{j}} \cap \overline{\Gamma^{i}}$ and $\operatorname{div}_{j}$ denotes the 2D-divergence computed on the face $\Gamma^{j}$. By density, this differential operator can be extended to less regular distributions and, in particular, to functionals in $\boldsymbol{H}_{\times}^{-\frac{1}{2}}(\Gamma)$. Moreover, (8) holds true in the appropriate sense. Thus, we set

$$
\boldsymbol{H}_{\times}^{-\frac{1}{2}}\left(\operatorname{div}_{\Gamma}, \Gamma\right):=\left\{\boldsymbol{\mu} \in \boldsymbol{H}_{\times}^{-\frac{1}{2}}(\Gamma), \operatorname{div}_{\Gamma} \boldsymbol{\mu} \in H^{-\frac{1}{2}}(\Gamma)\right\} .
$$

Finally, we denote by $\operatorname{curl}_{\Gamma}$ the operator adjoint to $\operatorname{div}_{\Gamma}$ with respect to the scalar product $\langle\cdot, \cdot\rangle_{\boldsymbol{\tau}, \Gamma}$, i.e.,

$$
\left\langle\operatorname{curl}_{\Gamma} q, \mathbf{p}\right\rangle_{\boldsymbol{\tau}, \Gamma}=\left\langle\operatorname{div}_{\Gamma} \mathbf{p}, q\right\rangle_{\frac{1}{2}, \Gamma}, \quad \mathbf{p} \in \boldsymbol{H}_{\times}^{-\frac{1}{2}}\left(\operatorname{div}_{\Gamma}, \Gamma\right), q \in H^{\frac{1}{2}}(\Gamma) .
$$

The following theorem proves that the space $\boldsymbol{H}_{\times}^{-\frac{1}{2}}\left(\operatorname{div}_{\Gamma}, \Gamma\right)$ fits the criterion announced at the beginning of this section:

Theorem 1. The operator $\gamma_{\mathbf{t}}: \boldsymbol{H}(\operatorname{curl} ; \Omega) \mapsto \boldsymbol{H}_{\times}^{-\frac{1}{2}}\left(\operatorname{div}_{\Gamma}, \Gamma\right)$ is continuous, surjective, and possesses a continuous right inverse.

Proof. See Theorem 4.4 in [18] for the case of Lipschitz polyhedra. The more general assertion for Lipschitz domains is shown in [20, Sect. 4].

In the case of Maxwell's equations the role of Cauchy data is played by $\gamma_{\mathbf{t}} \mathbf{e}$ and $\gamma_{\mathbf{t}} \mathbf{h}$. By the fundamental symmetry of electric and magnetic field, $\boldsymbol{H}_{\times}^{-\frac{1}{2}}\left(\operatorname{div}_{\Gamma}, \Gamma\right)$ is the right trace space for both fields. Everything is fitting, because this space is its own dual, as is confirmed by the following theorem [20, Lemma 5.6] 
Theorem 2 (Self-duality of $\boldsymbol{H}_{\times}^{-\frac{1}{2}}\left(\operatorname{div}_{\Gamma}, \Gamma\right)$ ). The pairing $\langle\cdot, \cdot\rangle_{\boldsymbol{\tau}, \Gamma}$ can be extended to a continuous bilinear form on $\boldsymbol{H}_{\times}^{-\frac{1}{2}}\left(\operatorname{div}_{\Gamma}, \Gamma\right)$. With respect to $\langle\cdot, \cdot\rangle_{\boldsymbol{\tau}, \Gamma}$ the space $\boldsymbol{H}_{\times}^{-\frac{1}{2}}\left(\operatorname{div}_{\Gamma}, \Gamma\right)$ becomes its own dual.

When we want to examine the convergence of boundary element methods quantitatively, extra smoothness of the functions to be approximated is indispensable. For any $s>\frac{1}{2}$, we define $\mathbf{H}_{-}^{s}(\Gamma):=\left\{\mathbf{u} \in \mathbf{L}_{\mathbf{t}}^{2}(\Gamma): \mathbf{u}_{\mid \Gamma^{j}} \in \boldsymbol{H}_{\mathbf{t}}^{s}\left(\Gamma^{j}\right)\right\}$ and $\boldsymbol{H}_{\times}^{s}(\Gamma):=\boldsymbol{H}_{\times}^{\frac{1}{2}}(\Gamma) \cap \mathbf{H}_{-}^{s}(\Gamma)$. The corresponding space of scalar functions will be denoted by $H_{-}^{s}(\Gamma)$. To characterize the smoothness we resort to the family of Hilbert spaces

$$
\boldsymbol{H}_{\times}^{s}\left(\operatorname{div}_{\Gamma}, \Gamma\right):= \begin{cases}\boldsymbol{H}_{\times}^{-\frac{1}{2}}\left(\operatorname{div}_{\Gamma}, \Gamma\right), & \text { if } s=-\frac{1}{2}, \\ \left\{\boldsymbol{\mu} \in \boldsymbol{H}_{\times}^{s}(\Gamma), \operatorname{div}_{\Gamma} \boldsymbol{\mu} \in H^{s}(\Gamma)\right\}, & \text { if }-\frac{1}{2}<s<\frac{1}{2}, \\ \left\{\boldsymbol{\mu} \in \boldsymbol{H}_{\times}^{s}(\Gamma), \operatorname{div}_{\Gamma} \boldsymbol{\mu} \in H_{-}^{s}(\Gamma)\right\}, & \text { if } s>\frac{1}{2} .\end{cases}
$$

As demonstrated in [16, Appendix 2], these spaces can be obtained through complex interpolation for $-\frac{1}{2} \leq s<\frac{1}{2}$. From this fact we conclude the following trace theorem (see [16]).

Theorem 3. The tangential trace mapping $\gamma_{\mathrm{t}}$ can be extended to a continuous mapping $\gamma_{\mathbf{t}}: \boldsymbol{H}^{s}(\mathbf{c u r l}, \Omega) \mapsto \boldsymbol{H}_{\times}^{s-\frac{1}{2}}\left(\operatorname{div}_{\Gamma}, \Gamma\right)$ for all $0 \leq s<1$.

\section{Maxwell versus Helmholtz}

There is a striking similarity between the electric wave equation (2) and the scalar Helmholtz equation

$$
-\operatorname{div}\left(\underline{\boldsymbol{\mu}}^{-1} \operatorname{grad} p\right)-\omega^{2} \epsilon p=0, \quad \text { in } \Omega_{s} \cup \Omega^{\prime} .
$$

In fact, the relationship between (2) and (14) runs much deeper than mere appearance: both equations emerge from a single equation for differential forms on $\mathbb{R}^{3}$, where (14) involves 0-forms, whereas (2) is the version for 1-forms [45, Sect. 2]. Hardly surprising, the theories of boundary integral equation methods for the related boundary value problems largely rely on the same principles. Nevertheless, the technical difficulties encountered in the treatment of the electric wave equation and related boundary element methods are significantly bigger than in the case of (14).

To appreciate what accounts for the fundamental difference between electromagnetism and acoustics, let us temporarily consider the variational source problem in a bounded Lipschitz domain $\Omega, c f$. [45, Sect. 5]. For (2) this reads: for $\mathbf{j} \in \boldsymbol{L}^{2}(\Omega)$ find $\mathbf{e} \in \boldsymbol{H}(\operatorname{curl} ; \Omega)$ such that for all $\mathbf{v} \in \boldsymbol{H}(\operatorname{curl} ; \Omega)$

$$
a_{M}(\mathbf{e}, \mathbf{v}):=\left(\underline{\boldsymbol{\mu}}^{-1} \operatorname{curl} \mathbf{e}, \mathbf{c u r l} \mathbf{v}\right)_{0}-\omega^{2}(\underline{\epsilon} \mathbf{e}, \mathbf{v})_{0}=-i \omega(\mathbf{j}, \mathbf{v})_{0},
$$


where $(u, v)_{0}:=\int_{\Omega} u v \mathrm{~d} \mathbf{x}$. The related problem for the Helmholtz equation and $f \in L^{2}(\Omega)$ seeks $p \in H^{1}(\Omega)$ such that

$$
a_{H}(p, q):=(\operatorname{grad} p, \operatorname{grad} q)_{0}-\omega^{2}(p, q)_{0}=(f, q)_{0} \quad \forall q \in H^{1}(\Omega) .
$$

Investigations of the convergence of Galerkin schemes for (16) usually centre on the concept of coercivity of the underlying bilinear form $a_{H}(\cdot, \cdot)$, that is, the fact that the zero order term is a compact perturbation of the second order term, the principal part, and that a Gårding inequality of the form

$$
\left|a_{H}(p, \bar{p})+c_{H}(p, \bar{p})\right| \geq C\|p\|_{H^{1}(\Omega)}^{2} \quad \forall p \in H^{1}(\Omega)
$$

holds with $C>0$ and a bilinear form $c_{H}(\cdot \cdot)$, which is compact in $H^{1}(\Omega)$. As has been demonstrated by Schatz [58], $c f$.also [62], this is the key to a priori asymptotic error estimates for Galerkin finite element methods. Evidently, we cannot expect an analogue of (17) from $a_{M}$. The blame lies with the infinite dimensional kernel of the curl-operator, which foils compactness of the imbedding $\boldsymbol{H}(\operatorname{curl} ; \Omega) \hookrightarrow \boldsymbol{L}^{2}(\Omega)$.

The issue of coercivity can also be discussed from the point of view of "energies": both acoustic and electromagnetic scattering are marked by an incessant conversion of energies. In acoustics, potential and kinetic energy of the fluid are converted into each other, in electromagnetism the same roles are played by the electric and magnetic energy. In acoustics the potential energy (with respect to the bounded control volume $\Omega$ ) is a compact perturbation of the kinetic energy ${ }^{1}$. Therefore we can clearly single out the Laplacian as the principal part of the Helmholtz operator. Conversely, in electromagnetism the electric and magnetic energies of a field are perfectly symmetric. Neither is a compact perturbation of the other. This means that no part of the electric wave equation is "principal". Formally speaking, the operator of the electric wave equation lacks the essential property of strong ellipticity. A concise summary is given in Table 1.

Table 1. Acoustics vs. electromagnetics in terms of dominant energies

\begin{tabular}{c|c} 
Acoustic wave equation & Electric wave equation \\
\hline$-\Delta p-\kappa^{2} p=0$ & $\operatorname{curl} \operatorname{curl} \mathbf{e}-\kappa^{2} \mathbf{e}=0$
\end{tabular}

Energies entering the Lagrangian:

\begin{tabular}{c|c}
$\begin{array}{c}\text { Kinetic "energy" } \int_{\Omega}|\operatorname{grad} p|^{2} \mathrm{dx} \\
\text { Potential "energy" } \int_{\Omega}|p|^{2} \mathrm{~d} \mathbf{x}\end{array}$ & $\begin{array}{c}\text { Magnetic "energy" } \int_{\Omega}|\operatorname{curl} \mathbf{e}|^{2} \mathrm{~d} \mathbf{x} \\
\text { Electric "energy" } \int_{\Omega}|\mathbf{e}|^{2} \mathrm{~d} \mathbf{x}\end{array}$ \\
$\begin{array}{c}\text { Potential energy a is compact } \\
\text { perturbation of kinetic energy }\end{array}$ & $\begin{array}{c}\text { Symmetry between electric } \\
\text { and magnetic quantities }\end{array}$
\end{tabular}

${ }^{1}$ Roles might be reversed depending on the formulation of the acoustic equations. 
The lack of a principal part can be overcome by the splitting of the fields into two components. One set of components, called the electric, will feature dominant electric energy. With the other set, the magnetic quantities, the situation is reversed. This will promote either curl curl or Id to the role of a principal part. As a consequence, on each component the electric wave equation should be amenable to the same treatment as the Helmholtz equation. In the context of electromagnetic problems the splitting idea has been pioneered by Nédélec and was first applied to integral operators in [37]. Since then it has emerged as a very powerful theoretical tool, see $[6,19,26]$ and, in particular, the monograph [53]. Three features of a splitting prove essential:

1. one subspace in the splitting agrees with the kernel of curl,

2. the compact embedding of the other subspace (complement space) into $\boldsymbol{L}^{2}(\Omega)$,

3. the extra smoothness of vector-fields in the complement space.

This makes it possible to opt for the Helmholtz-type regular splitting provided by the next lemma.

Lemma 1 (Regular decomposition lemma). There exists a continuous projector $\mathrm{R}: \boldsymbol{H}(\operatorname{curl} ; \Omega) \mapsto \boldsymbol{H}^{1}(\Omega) \cap \boldsymbol{H}(\operatorname{div} 0 ; \Omega)$ such that $\operatorname{Ker}(\mathrm{R})=\boldsymbol{H}(\operatorname{curl} 0 ; \Omega)$.

The proof is given in [45, Sect. 2.4] and makes use of the existence of regular vector potentials, $c f$. Lemma 3.5 in [7].

Evidently, the three requirements are satisfied by the decomposition

$$
\boldsymbol{H}(\operatorname{curl} ; \Omega)=\mathcal{X}(\Omega) \oplus \mathcal{N}(\Omega), \boldsymbol{X}(\Omega):=\mathrm{R}(\boldsymbol{H}(\operatorname{curl} ; \Omega)) \subset \boldsymbol{H}^{1}(\Omega),
$$

where we write $\mathcal{N}(\Omega):=\boldsymbol{H}(\operatorname{curl} 0 ; \Omega)$. The continuity of the projectors guarantees the stability of this decomposition. Now, we can consider the variational problem (15) with respect to (18): thanks to the compact embedding of $\boldsymbol{H}^{1}(\Omega)$ into $\boldsymbol{L}^{2}(\Omega)$ we see that the second term of the bilinear form

$$
\left(\mathbf{e}_{\perp}, \mathbf{v}_{\perp}\right) \mapsto\left(\underline{\boldsymbol{\mu}}^{-1} \operatorname{curl} \mathbf{e}_{\perp}, \operatorname{curl} \mathbf{v}_{\perp}\right)_{0}-\omega^{2}\left(\underline{\epsilon} \mathbf{e}_{\perp}, \mathbf{v}_{\perp}\right)_{0}, \quad \mathbf{e}_{\perp}, \mathbf{v}_{\perp} \in \mathcal{X}(\Omega),
$$

is a compact perturbation of the first: $a_{M}$ is coercive on $\mathcal{X}(\Omega)$. Coercivity on $\mathcal{N}(\Omega)$ is trivial. In addition, terms like

$$
\left(\mathbf{e}_{0}, \mathbf{v}_{\perp}\right) \mapsto\left(\underline{\epsilon} \mathbf{e}_{0}, \mathbf{v}_{\perp}\right)_{0}, \quad \mathbf{e}_{0} \in \mathcal{N}(\Omega), \mathbf{v}_{\perp} \in \mathcal{X}(\Omega),
$$

which effect the coupling of $\mathcal{X}(\Omega)$ and $\mathcal{N}(\Omega)$ with respect to $a_{M}$, can also be dismissed as compact perturbations. Using the isomorphism $\mathrm{X}_{\Omega}: \boldsymbol{H}(\operatorname{curl} ; \Omega) \mapsto$ $\boldsymbol{H}(\operatorname{curl} ; \Omega)$, defined by $\mathrm{X}_{\Omega}:=\mathrm{R}-\mathrm{Z}$, where $\mathrm{Z}:=\mathrm{Id}-\mathrm{R}$ is the complementary projector to R, to "flip signs", we arrive at

$$
\left|a_{M}\left(\mathbf{u}, \mathrm{X}_{\Omega} \overline{\mathbf{u}}\right)-\mathbf{c}_{M}(\mathbf{u}, \overline{\mathbf{u}})\right| \geq C\|\mathbf{u}\|_{\boldsymbol{H}(\mathbf{c u r l} ; \Omega)}^{2} \quad \forall \mathbf{u} \in \boldsymbol{H}(\operatorname{curl} ; \Omega),
$$

with some $C>0$ and a compact bilinear form $\mathbf{c}_{M}$ on $\boldsymbol{H}(\mathbf{c u r l} ; \Omega)$. The generalized Garding inequality (19) is the crucial assumption in the following fundamental theorem: 
Theorem 4. If a bilinear form a : $V \times V \mapsto \mathbb{C}$ on a reflexive Banach space $V$ satisfies

$$
\left|a\left(u, \mathrm{X}_{\Omega} \bar{u}\right)-c(u, \bar{u})\right| \geq C\|u\|_{V}^{2} \quad \forall u \in V
$$

with $C>0$, a compact bilinear form $c: V \times V \mapsto \mathbb{C}$, and an isomorphism $\mathrm{X}_{\Omega}: V \mapsto V$, then the associated operator $A: V \mapsto V^{\prime}$ is Fredholm with index 0 .

In particular, for a bilinear form meeting the requirements of the theorem, injectivity of the associated operator implies its surjectivity by the Fredholm alternative [11].

It is hardly surprising that the splitting idea also plays a pivotal role in the analysis of boundary integral equations arising from the electric wave equation. Here, it is applied to the trace space $\boldsymbol{H}_{\times}^{-\frac{1}{2}}\left(\operatorname{div}_{\Gamma}, \Gamma\right)$ :

Lemma 2. There exists a projection $\mathrm{R}^{\Gamma}: \boldsymbol{H}_{\times}^{-\frac{1}{2}}\left(\operatorname{div}_{\Gamma}, \Gamma\right) \mapsto \boldsymbol{H}_{\times}^{\frac{1}{2}}(\Gamma)$ such that $\operatorname{Ker}\left(\mathrm{R}^{\Gamma}\right)=\boldsymbol{H}_{\times}^{-\frac{1}{2}}\left(\operatorname{div}_{\Gamma} 0, \Gamma\right)$ and

$$
\left\|\mathrm{R}^{\Gamma} \boldsymbol{\mu}\right\|_{H_{\times}^{\frac{1}{2}}(\Gamma)} \leq C\left\|\operatorname{div}_{\Gamma} \boldsymbol{\mu}\right\|_{H^{-\frac{1}{2}}(\Gamma)} .
$$

Proof. Pick $\boldsymbol{\lambda} \in \boldsymbol{H}_{\times}^{-\frac{1}{2}}\left(\operatorname{div}_{\Gamma}, \Gamma\right)$ and set $\mu:=\operatorname{div}_{\Gamma} \boldsymbol{\lambda} \in H^{-\frac{1}{2}}(\Gamma)$. Solve the Neumann problem

$$
w \in H^{1}\left(\Omega_{s}\right) / \mathbb{R}: \quad \Delta w=0 \quad \text { in } \Omega_{s}, \quad \gamma_{\mathbf{n}}^{-} \operatorname{grad} w=\mu \quad \text { on } \Gamma .
$$

We find that $\mathbf{v}:=\operatorname{grad} w \in \boldsymbol{H}\left(\operatorname{div} 0 ; \Omega_{s}\right)$. Using Lemma 3.5 in [7], there exists $\mathbf{w} \in \boldsymbol{H}^{1}\left(\Omega_{s}\right)$ such that $\mathbf{v}=\operatorname{curl} \mathbf{w}, \operatorname{div} \mathbf{w}=0$. This defines an operator $\mathbf{J}$ : $H^{-\frac{1}{2}}(\Gamma) \mapsto \boldsymbol{H}^{1}\left(\Omega_{s}\right)$ by $\mathrm{J} \mu:=\mathbf{w}$. Its continuity is elementary

$$
\|\mathrm{J} \mu\|_{\boldsymbol{H}^{1}\left(\Omega_{s}\right)} \leq C\|\mathbf{v}\|_{\boldsymbol{L}^{2}\left(\Omega_{s}\right)} \leq C\|\mu\|_{H^{-\frac{1}{2}(\Gamma)}}
$$

and inherited by the mapping $\mathrm{R}^{\Gamma}:=\gamma_{\mathbf{t}} \circ \mathrm{J} \circ \operatorname{div}_{\Gamma}: \boldsymbol{H}_{\times}^{-\frac{1}{2}}\left(\operatorname{div}_{\Gamma}, \Gamma\right) \mapsto \boldsymbol{H}_{\times}^{\frac{1}{2}}(\Gamma)$. Moreover, we see that $\operatorname{div}_{\Gamma} \mathrm{R}^{\Gamma} \boldsymbol{\lambda}=\gamma_{\mathbf{n}}^{-} \operatorname{curl} \mathbf{L v}=\gamma_{\mathbf{n}}^{-} \mathbf{v}=\operatorname{div}_{\Gamma} \boldsymbol{\lambda}$.

As before, the projector complementary to $\mathrm{R}^{\Gamma}$ will be denoted by $\mathrm{Z}^{\Gamma}$. We arrive at a stable decomposition of the trace space

$$
\boldsymbol{H}_{\times}^{-\frac{1}{2}}\left(\operatorname{div}_{\Gamma}, \Gamma\right):=\mathcal{X}(\Gamma) \oplus \mathcal{N}(\Gamma),
$$

where $\mathcal{X}(\Gamma):=\mathrm{R}^{\Gamma}\left(\boldsymbol{H}_{\times}^{-\frac{1}{2}}\left(\operatorname{div}_{\Gamma}, \Gamma\right)\right)$ and $\mathcal{N}(\Gamma)=\boldsymbol{H}_{\times}^{-\frac{1}{2}}\left(\operatorname{div}_{\Gamma} 0, \Gamma\right)$. Both components inherit the norm of $\boldsymbol{H}_{\times}^{-\frac{1}{2}}\left(\operatorname{div}_{\Gamma}, \Gamma\right)$.

Corollary 1. The embedding $\mathcal{X}(\Gamma) \hookrightarrow \boldsymbol{L}_{\mathbf{t}}^{2}(\Gamma)$ is compact. 
It is illuminating to give a physical interpretation of the decomposition. First, view $\boldsymbol{H}_{\times}^{-\frac{1}{2}}\left(\operatorname{div}_{\Gamma}, \Gamma\right)$ as a space of tangential components of electric fields. Then, we encounter traces of "static" irrotational fields in $\mathcal{N}(\Gamma)$, whereas traces of "dynamic" field components, whose curls do not vanish, are associated with $\mathcal{X}(\Gamma)$. By Faraday's law the latter are linked with magnetic fields. All in all, we can attribute an "electric nature" to the space $\mathcal{N}(\Gamma)$, and a "magnetic nature" to $\mathcal{X}(\Gamma)$. The arguments are simply reversed when considering magnetic traces $\gamma_{N} \mathbf{e}$, because given the absence of source currents the magnetic field is irrotational in the stationary case. This means that components of $\gamma_{N} \mathbf{e}$ that belong to $\mathcal{N}(\Gamma)$ are "magnetic", whereas components in $\mathcal{X}(\Gamma)$ are "electric", $c f$. Table 2.

Table 2. Physical nature of components occurring in the splitting of fields and traces

\begin{tabular}{|c|c|c|c|}
\hline Field & Space & $\begin{array}{c}\text { Magnetic } \\
\text { components }\end{array}$ & $\begin{array}{c}\text { Electric } \\
\text { components }\end{array}$ \\
\hline $\mathbf{e}$ & $\boldsymbol{H}(\mathbf{c u r l} ; \Omega)$ & $\mathcal{X}(\Omega)$ & $\mathcal{N}(\Omega)$ \\
$\gamma_{\mathbf{t}} \mathbf{e}$ & $\boldsymbol{H}_{\times}^{-\frac{1}{2}}\left(\operatorname{div}_{\Gamma}, \Gamma\right)$ & $\mathcal{X}(\Gamma)$ & $\mathcal{N}(\Gamma)$ \\
$\gamma_{N} \mathbf{e}$ & $\boldsymbol{H}_{\times}^{-\frac{1}{2}}\left(\operatorname{div}_{\Gamma}, \Gamma\right)$ & $\mathcal{N}(\Gamma)$ & $\mathcal{X}(\Gamma)$ \\
\hline
\end{tabular}

\section{Representation Formulas}

In this section we start from the electric wave equation (2) in the air region $\Omega^{\prime}$, where $\underline{\boldsymbol{\mu}}$ and $\underline{\boldsymbol{\epsilon}}$ can be regarded as scalar constants $\mu_{0}$ and $\epsilon_{0}$. Then, the partial differential equation (2) can be recast as

$$
\text { curl curle }-\kappa^{2} \mathbf{e}=0
$$

The constant $\kappa:=\omega \sqrt{\epsilon_{0} \mu_{0}}>0$ is called the wave number, because $\kappa / 2 \pi$ tells us the number of wavelengths per unit length. Henceforth, $\kappa$ will stand for a fixed positive wave number ${ }^{2}$.

Definition 2. A distribution e $\in \boldsymbol{H}_{\mathrm{loc}}\left(\mathbf{c u r l}^{2}, \Omega\right)$ is called a Maxwell solution on some generic domain $\Omega$, if it satisfies (22) in $\Omega$, and the Silver-Müller radiation conditions at $\infty$, if $\Omega$ is not bounded.

It is our objective to derive a boundary integral representation formula for Maxwell solutions. In order to handle transmission conditions in the calculus of distributions, we introduce currents, that is, distributions supported on $\Gamma$. For a function $\varphi \in$ $H^{-\frac{1}{2}}(\Gamma)$, a tangential vector-field $\boldsymbol{\xi} \in \boldsymbol{H}_{\times}^{-1}(\Gamma)$, and test functions $\Phi \in \mathcal{D}\left(\mathbb{R}^{3}\right)$, $\boldsymbol{\Phi} \in \mathcal{D}\left(\mathbb{R}^{3}\right):=\left(\mathcal{D}\left(\mathbb{R}^{3}\right)\right)^{3}$, we define

\footnotetext{
${ }^{2}$ We point out that all considerations remain true if $\operatorname{Im} \kappa>0$ (lossy media)
} 


$$
\left(\varphi \delta_{\Gamma}\right)(\Phi):=\langle\varphi, \gamma \Phi\rangle_{\frac{1}{2}, \Gamma}, \quad\left(\boldsymbol{\xi} \boldsymbol{\delta}_{\Gamma}\right)(\boldsymbol{\Phi}):=\left\langle\boldsymbol{\xi}, \gamma_{\mathbf{t}} \boldsymbol{\Phi}\right\rangle_{\boldsymbol{\tau}, \Gamma}=\langle\boldsymbol{\xi}, \gamma \boldsymbol{\Phi}\rangle_{-1, \Gamma}
$$

Recall the notation used in the introduction: the superscripts - and + tag traces onto $\Gamma$ from $\Omega_{s}$ and $\Omega^{\prime}$ respectively. Now, in the sense of distributions, integration by parts yields, $c f$. [16, Section 2.3],

$$
\begin{array}{llrl}
\text { for } \mathbf{u} \in \boldsymbol{H}_{\mathrm{loc}}\left(\operatorname{div} ; \Omega_{s} \cup \Omega^{\prime}\right) & : & \operatorname{div} \mathbf{u}=\operatorname{div} \mathbf{u}_{\mid \Omega_{s} \cup \Omega^{\prime}}+\left[\gamma_{\mathbf{n}}\right]_{\Gamma}(\mathbf{u}) \delta_{\Gamma}, \\
\text { for } \mathbf{u} \in \boldsymbol{H}_{\mathrm{loc}}\left(\operatorname{curl} ; \Omega_{s} \cup \Omega^{\prime}\right) & : & \operatorname{curl} \mathbf{u}=\operatorname{curl} \mathbf{u}_{\mid \Omega_{s} \cup \Omega^{\prime}}-\left[\gamma_{\mathbf{t}}\right]_{\Gamma}(\mathbf{u}) \boldsymbol{\delta}_{\Gamma}, \\
\text { for } \boldsymbol{\xi} \in \boldsymbol{H}_{\times}^{-\frac{1}{2}}\left(\operatorname{div}_{\Gamma}, \Gamma\right) & : & \operatorname{div}\left(\boldsymbol{\xi} \boldsymbol{\delta}_{\Gamma}\right)=\left(\operatorname{div}_{\Gamma} \boldsymbol{\xi}\right) \delta_{\Gamma}
\end{array}
$$

For the sake of brevity, we have used the jump operator $[\cdot]_{\Gamma}$ defined by $[\gamma]_{\Gamma}:=$ $\gamma^{+}-\gamma^{-}$for some trace $\gamma$ onto $\Gamma$. For notational simplicity it is also useful to resort to the average $\{\gamma\}_{\Gamma}=\frac{1}{2}\left(\gamma^{+}+\gamma^{-}\right)$. Both operators can only be applied to functions defined in $\Omega_{s} \cup \Omega^{\prime}$. Moreover, we set $\gamma_{N}^{ \pm}:=\kappa^{-1} \gamma_{\mathbf{t}}^{ \pm} \circ$ curl.

Now, let $\mathbf{u}$ be a Maxwell solution in $\Omega_{s} \cup \Omega^{\prime}$, which, of course, satisfies $\operatorname{div} \mathbf{u}=0$ in $\Omega_{s} \cup \Omega^{\prime}$. Then the following identity holds in the sense of distributions,

$$
\begin{aligned}
-\Delta \mathbf{u}- & \kappa^{2} \mathbf{u}=\operatorname{curl} \operatorname{curl} \mathbf{u}-\operatorname{grad} \operatorname{div} \mathbf{u}-\kappa^{2} \mathbf{u} \\
= & \operatorname{curl}\left(\operatorname{curl} \mathbf{u}_{\mid \Omega_{s} \cup \Omega^{\prime}}-\left[\gamma_{\mathbf{t}}\right]_{\Gamma}(\mathbf{u}) \boldsymbol{\delta}_{\Gamma}\right)-\operatorname{grad}\left(\left[\gamma_{\mathbf{n}}\right]_{\Gamma}(\mathbf{u}) \delta_{\Gamma}\right)-\kappa^{2} \mathbf{u} \\
= & \operatorname{curl} \operatorname{curl} \mathbf{u}_{\mid \Omega_{s} \cup \Omega^{\prime}}-\kappa\left[\gamma_{N}\right]_{\Gamma}(\mathbf{u}) \boldsymbol{\delta}_{\Gamma}-\operatorname{curl}\left(\left[\gamma_{\mathbf{t}}\right]_{\Gamma}(\mathbf{u}) \boldsymbol{\delta}_{\Gamma}\right)- \\
& \quad-\operatorname{grad}\left(\left[\gamma_{\mathbf{n}}\right]_{\Gamma}(\mathbf{u}) \delta_{\Gamma}\right)-\kappa^{2} \mathbf{u} \\
= & -\kappa\left[\gamma_{N}\right]_{\Gamma}(\mathbf{u}) \boldsymbol{\delta}_{\Gamma}-\operatorname{curl}\left(\left[\gamma_{\mathbf{t}}\right]_{\Gamma}(\mathbf{u}) \boldsymbol{\delta}_{\Gamma}\right)-\operatorname{grad}\left(\left[\gamma_{\mathbf{n}}\right]_{\Gamma}(\mathbf{u}) \delta_{\Gamma}\right)
\end{aligned}
$$

As far as the differential operator curl curl $-\kappa^{2}$ Id is concerned, the integration by parts formula (11) suggests the distinction between Dirichlet trace $\gamma_{\mathbf{t}}$ and Neumann trace $\gamma_{N}$. The trace $\gamma_{N}$ can be labelled "magnetic", because it actually retrieves the tangential trace of the magnetic field solution. It has much in common with the Neumann trace operator $\gamma_{\mathbf{n}} \circ$ grad for the Helmholtz equation: for instance, it fails to be defined on $\boldsymbol{H}_{\text {loc }}\left(\operatorname{curl} ; \Omega_{s} \cup \Omega^{\prime}\right)$, but the weak definition

$$
-\frac{1}{\kappa} \int_{\Omega} \operatorname{curl} \mathbf{u} \cdot \operatorname{curl} \mathbf{v}-\operatorname{curl} \operatorname{curl} \mathbf{u} \cdot \mathbf{v} \mathrm{d} \mathbf{x}=\left\langle\gamma_{N} \mathbf{u}, \gamma_{\mathbf{t}} \mathbf{v}\right\rangle_{\boldsymbol{\tau}, \Gamma}
$$

$\mathbf{v} \in \mathcal{D}\left(\mathbb{R}^{3}\right)$, renders it meaningful on $\boldsymbol{H}_{\text {loc }}\left(\mathbf{c u r l}^{2}, \Omega_{s} \cup \Omega^{\prime}\right)$ [46, Lemma 3.3]:

Lemma 3. The trace $\gamma_{N}$ furnishes a continuous and surjective mapping $\gamma_{N}$ : $\boldsymbol{H}_{\mathrm{loc}}\left(\operatorname{curl}^{2}, \Omega^{\prime} \cup \Omega_{s}\right) \mapsto \boldsymbol{H}_{\times}^{-\frac{1}{2}}\left(\operatorname{div}_{\Gamma}, \Gamma\right)$.

Definition 3. Pairs $(\boldsymbol{\zeta}, \boldsymbol{\mu}) \in \boldsymbol{H}_{\times}^{-\frac{1}{2}}\left(\operatorname{div}_{\Gamma}, \Gamma\right) \times \boldsymbol{H}_{\times}^{-\frac{1}{2}}\left(\operatorname{div}_{\Gamma}, \Gamma\right)$ are called interiorlexterior Maxwell Cauchy data, if there is a Maxwell solution $\mathbf{u}$ in $\Omega_{s}$ and $\Omega^{\prime}$, respectively, such that $\boldsymbol{\zeta}=\gamma_{\mathbf{t}}^{ \pm} \mathbf{u}, \boldsymbol{\mu}=\gamma_{N}^{ \pm} \mathbf{u}$.

We know from [31, Theorem 6.7] that the Cartesian components of Maxwell solutions will satisfy the Sommerfeld radiation condition and the scalar Helmholtz 
equation in $\Omega_{s} \cup \Omega^{\prime}$. Using the results from [52, Ch. 9], we can apply componentwise convolution with the outgoing fundamental solution of the Helmholtz equation $E_{\kappa}(\mathbf{x}):=\exp (i \kappa|\mathbf{x}|) / 4 \pi|\mathbf{x}|, \mathbf{x} \neq 0$, and we find that almost everywhere in $\mathbb{R}^{3}$ the components of $\mathbf{u}=\left(u_{1}, u_{2}, u_{3}\right)^{T}$ satisfy

$$
\begin{aligned}
u_{j}(\mathbf{x})= & -\kappa\left(\left[\gamma_{N}\right]_{\Gamma}(\mathbf{u}) \boldsymbol{\delta}_{\Gamma}\right)\left(E_{\kappa}(\mathbf{x}-\cdot) \mathbf{e}_{j}\right)-\left(\left[\gamma_{\mathbf{t}}\right]_{\Gamma}(\mathbf{u}) \boldsymbol{\delta}_{\Gamma}\right)\left(\operatorname{curl}\left(E_{\kappa}(\mathbf{x}-\cdot) \mathbf{e}_{j}\right)\right)+ \\
& +\left(\left[\gamma_{\mathbf{n}}\right]_{\Gamma}(\mathbf{u}) \delta_{\Gamma}\right)\left(\operatorname{div}\left(E_{\kappa}(\mathbf{x}-\cdot) \mathbf{e}_{j}\right)\right), \quad j=1,2,3 .
\end{aligned}
$$

Using $\operatorname{grad}_{\mathbf{x}} E_{\kappa}(\mathbf{x}-\mathbf{y})=-\operatorname{grad}_{\mathbf{y}} E_{\kappa}(\mathbf{x}-\mathbf{y})$, we arrive at the famous StrattonChu representation formula for the electric field in $\Omega_{s} \cup \Omega^{\prime}$ [60], $c f$.[31, Sect. 6.2], [53, Sect. 5.5], [24, Ch. 3, Sect. 1.3.2],

$$
\mathbf{u}=-\kappa \boldsymbol{\Psi}_{\mathbf{V}}^{\kappa}\left(\left[\gamma_{N}\right]_{\Gamma}(\mathbf{u})\right)-\operatorname{curl} \boldsymbol{\Psi}_{\mathbf{V}}^{\kappa}\left(\left[\gamma_{\mathbf{t}}\right]_{\Gamma}(\mathbf{u})\right)-\operatorname{grad} \Psi_{V}^{\kappa}\left(\left[\gamma_{\mathbf{n}}\right]_{\Gamma}(\mathbf{u})\right) .
$$

Here, $\boldsymbol{\Psi}_{\mathbf{V}}^{\kappa}(\cdot)$ and $\Psi_{V}^{\kappa}(\cdot)$ are potentials, that is, mappings of boundary data to analytic functions defined everywhere off the boundary. In detail, $\Psi_{V}^{\kappa}$ and $\boldsymbol{\Psi}_{\mathbf{V}}^{\kappa}$ are the scalar and vectorial single layer potential, whose integral representation is given by $(\mathbf{x} \notin \Gamma)$

$$
\Psi_{V}^{\kappa}(\phi)(\mathbf{x}):=\int_{\Gamma} \phi(\mathbf{y}) E_{\kappa}(\mathbf{x}-\mathbf{y}) d S(\mathbf{y}), \Psi_{\mathbf{V}}^{\kappa}(\boldsymbol{\mu})(\mathbf{x}):=\int_{\Gamma} \boldsymbol{\mu}(\mathbf{y}) E_{\kappa}(\mathbf{x}-\mathbf{y}) d S(\mathbf{y}) .
$$

A simplification of (24) is possible by observing that, by (8)

$$
\operatorname{div}_{\Gamma}\left(\gamma_{N}^{ \pm} \mathbf{u}\right)=\kappa^{-1} \gamma_{\mathbf{n}}^{ \pm}(\operatorname{curl} \operatorname{curl} \mathbf{u})=\kappa\left(\gamma_{\mathbf{n}}^{ \pm} \mathbf{u}\right) \quad \text { in } H^{-\frac{1}{2}}(\Gamma)
$$

This enables us to get rid of the normal components trace in (24). We end up with the pointwise identity

$$
\mathbf{u}(\mathbf{x})=-\boldsymbol{\Psi}_{D L}^{\kappa}\left(\left[\gamma_{\mathbf{t}}\right]_{\Gamma}(\mathbf{u})\right)(\mathbf{x})-\boldsymbol{\Psi}_{S L}^{\kappa}\left(\left[\gamma_{N}\right]_{\Gamma}(\mathbf{u})\right)(\mathbf{x}), \quad \mathbf{x} \in \Omega_{s} \cup \Omega^{\prime}
$$

where we have introduced the (electric) Maxwell single layer potential according to

$$
\boldsymbol{\Psi}_{S L}^{\kappa}(\boldsymbol{\mu})(\mathbf{x}):=\kappa \boldsymbol{\Psi}_{\mathbf{V}}^{\kappa}(\boldsymbol{\mu})(\mathbf{x})+\frac{1}{\kappa} \operatorname{grad}_{\mathbf{x}} \Psi_{V}^{\kappa}\left(\operatorname{div}_{\Gamma} \boldsymbol{\mu}\right)(\mathbf{x}), \quad \mathbf{x} \notin \Gamma
$$

and the (electric) Maxwell double layer potential

$$
\boldsymbol{\Psi}_{D L}^{\kappa}(\boldsymbol{\mu})(\mathbf{x}):=\operatorname{curl}_{\mathbf{x}} \boldsymbol{\Psi}_{\mathbf{V}}^{\kappa}(\boldsymbol{\mu})(\mathbf{x}), \quad \mathbf{x} \notin \Gamma
$$

We have chosen these names in order to underscore the similarity of (26) with the representation formula for solutions of the Helmholtz equation [31, Sect. 3.1], [52, Ch. 9].

Next, we aim to fit the potentials into the functional framework devised in Sect. 2. To this end we have to show that the potentials $\boldsymbol{\Psi}_{D L}^{\kappa}$ and $\boldsymbol{\Psi}_{S L}^{\kappa}$ are continuous operators between the canonical function spaces for traces and the appropriate function spaces for Maxwell solutions. An important result from [19] and [33] will be useful 
Lemma 4. The single layer potentials $\Psi_{V}^{\kappa}$ and $\boldsymbol{\Psi}_{\mathbf{V}}^{\kappa}$ give rise to continuous mappings

$$
\Psi_{V}^{\kappa}: H^{-\frac{1}{2}+s}(\Gamma) \mapsto H_{\mathrm{loc}}^{1+s}\left(\mathbb{R}^{3}\right), \quad \Psi_{\mathbf{V}}^{\kappa}: \boldsymbol{H}_{\times}^{-\frac{1}{2}+s}(\Gamma) \mapsto \boldsymbol{H}_{\mathrm{loc}}^{1+s}\left(\mathbb{R}^{3}\right)
$$

for any $s,-\frac{1}{2}<s \leq \frac{1}{2}$.

¿From this lemma we conclude that both $\boldsymbol{\Psi}_{S L}^{\kappa}$ and $\boldsymbol{\Psi}_{D L}^{\kappa}$ are well defined for arguments in the trace space $\boldsymbol{H}_{\times}^{-\frac{1}{2}}\left(\operatorname{div}_{\Gamma}, \Gamma\right)$. To gain deeper insights into the continuity property of the Maxwell single layer and double layer potentials, we have to make some preparations, $c f$. Lemma 2.3 in [51].

Lemma 5. For $\boldsymbol{\mu} \in \boldsymbol{H}_{\times}^{-\frac{1}{2}}\left(\operatorname{div}_{\Gamma}, \Gamma\right)$ we have $\operatorname{div} \boldsymbol{\Psi}_{\mathbf{V}}^{\kappa}(\boldsymbol{\mu})=\Psi_{V}^{\kappa}\left(\operatorname{div}_{\Gamma} \boldsymbol{\mu}\right)$ in $\boldsymbol{L}^{2}\left(\mathbb{R}^{3}\right)$.

By definition and curl $\circ \operatorname{grad}=0$, it is immediate that $\operatorname{curl} \circ \boldsymbol{\Psi}_{S L}^{\kappa}=\kappa \boldsymbol{\Psi}_{D L}^{\kappa}$ on $\boldsymbol{H}_{\times}^{-\frac{1}{2}}\left(\operatorname{div}_{\Gamma}, \Gamma\right)$. On the other hand, using the previous lemma, we get for $\boldsymbol{\mu} \in$ $\boldsymbol{H}_{\times}^{-\frac{1}{2}}\left(\operatorname{div}_{\Gamma}, \Gamma\right)$

$$
\begin{aligned}
\operatorname{curl} \boldsymbol{\Psi}_{D L}^{\kappa}(\boldsymbol{\mu}) & =\operatorname{curl} \operatorname{curl} \boldsymbol{\Psi}_{\mathbf{V}}^{\kappa}(\boldsymbol{\mu})=(-\boldsymbol{\Delta}+\operatorname{grad} \operatorname{div}) \boldsymbol{\Psi}_{\mathbf{V}}^{\kappa}(\boldsymbol{\mu}) \\
& =\kappa^{2} \boldsymbol{\Psi}_{\mathbf{V}}^{\kappa}(\boldsymbol{\mu})+\operatorname{grad} \Psi_{V}^{\kappa}\left(\operatorname{div}_{\Gamma} \boldsymbol{\mu}\right)=\kappa \boldsymbol{\Psi}_{S L}^{\kappa}(\boldsymbol{\mu}) .
\end{aligned}
$$

Here, we have used $-\boldsymbol{\Delta} \boldsymbol{\Psi}_{\mathbf{V}}^{\kappa}(\boldsymbol{\mu})=\kappa^{2} \boldsymbol{\Psi}_{\mathbf{V}}^{\kappa}(\boldsymbol{\mu})$. Altogether, both potentials are Maxwell solutions, that is, for $\boldsymbol{\mu} \in \boldsymbol{H}_{\times}^{-\frac{1}{2}}\left(\operatorname{div}_{\Gamma}, \Gamma\right)$ they fulfil

$$
\left(\operatorname{curl} \operatorname{curl}-\kappa^{2} \operatorname{Id}\right) \Psi_{S L}^{\kappa}(\boldsymbol{\mu})=0 \quad, \quad\left(\operatorname{curl} \operatorname{curl}-\kappa^{2} \mathrm{Id}\right) \boldsymbol{\Psi}_{D L}^{\kappa}(\boldsymbol{\mu})=0,
$$

off the boundary $\Gamma$ in a pointwise sense, and, globally, in $\boldsymbol{L}_{\text {loc }}^{2}\left(\mathbb{R}^{3}\right)$. In addition, they comply with the Silver-Müller radiation conditions. From these relationships and Lemma 4 we infer the desired continuity properties.

Theorem 5. The following mappings are continuous

$$
\begin{aligned}
& \boldsymbol{\Psi}_{S L}^{\kappa}: \boldsymbol{H}_{\times}^{-\frac{1}{2}}\left(\operatorname{div}_{\Gamma}, \Gamma\right) \mapsto \boldsymbol{H}_{\mathrm{loc}}\left(\mathbf{c u r l}^{2}, \Omega_{s} \cup \Omega^{\prime}\right) \cap \boldsymbol{H}_{\mathrm{loc}}\left(\operatorname{div} 0 ; \Omega_{s} \cup \Omega^{\prime}\right), \\
& \boldsymbol{\Psi}_{D L}^{\kappa}: \boldsymbol{H}_{\times}^{-\frac{1}{2}}\left(\operatorname{div}_{\Gamma}, \Gamma\right) \mapsto \boldsymbol{H}_{\mathrm{loc}}\left(\operatorname{curl}^{2}, \Omega_{s} \cup \Omega^{\prime}\right) \cap \boldsymbol{H}_{\mathrm{loc}}\left(\operatorname{div} 0 ; \Omega_{s} \cup \Omega^{\prime}\right)
\end{aligned}
$$

Now, we are in a position to extract the desired identities from (26).

Theorem 6 (Stratton-Chu representation formula). Any Maxwell solution $\mathbf{u}$ in $\Omega_{s}$ possesses the representation

$$
\mathbf{u}=\boldsymbol{\Psi}_{D L}^{\kappa}\left(\gamma_{\mathbf{t}}^{-} \mathbf{u}\right)+\boldsymbol{\Psi}_{S L}^{\kappa}\left(\gamma_{N}^{-} \mathbf{u}\right) \quad \text { in } \boldsymbol{H}\left(\operatorname{curl}^{2}, \Omega_{s}\right)
$$

If $\mathbf{u}$ is a Maxwell solution in $\Omega^{\prime}$ that satisfies the Silver-Müller radiation conditions, it can be written as

$$
\mathbf{u}=-\boldsymbol{\Psi}_{D L}^{\kappa}\left(\gamma_{\mathbf{t}}^{+} \mathbf{u}\right)-\boldsymbol{\Psi}_{S L}^{\kappa}\left(\gamma_{N}^{+} \mathbf{u}\right) \quad \text { in } \boldsymbol{H}_{\mathrm{loc}}\left(\operatorname{curl}^{2}, \Omega^{\prime}\right)
$$




\section{Boundary Integral Operators}

By Lemma 3, Theorem 5 provides the foundation for applying both the Dirichlet trace $\gamma_{\mathbf{t}}$ and the Neumann trace $\gamma_{N}$ to the potentials $\boldsymbol{\Psi}_{S L}^{\kappa}$ and $\boldsymbol{\Psi}_{D L}^{\kappa}$. This is the canonical way of constructing boundary integral operators [52, Chapter 7]. In the case of second order elliptic problems, four different boundary integral operators arise. Yet, due to the fact that $\operatorname{curl} \circ \boldsymbol{\Psi}_{S L}^{\kappa}=\kappa \boldsymbol{\Psi}_{D L}^{\kappa}, \operatorname{curl} \circ \boldsymbol{\Psi}_{D L}^{\kappa}=\kappa \boldsymbol{\Psi}_{S L}^{\kappa}$ implies

$$
\gamma_{N}^{ \pm} \boldsymbol{\Psi}_{S L}^{\kappa}=\gamma_{\mathbf{t}}^{ \pm} \boldsymbol{\Psi}_{D L}^{\kappa}, \gamma_{N}^{ \pm} \boldsymbol{\Psi}_{D L}^{\kappa}=\gamma_{\mathbf{t}}^{ \pm} \boldsymbol{\Psi}_{S L}^{\kappa}
$$

two different boundary integral operators are sufficient for electromagnetic scattering: we obtain the boundary integral operators

$$
\mathbf{S}_{\kappa}:=\left\{\gamma_{\mathbf{t}}\right\}_{\Gamma} \circ \boldsymbol{\Psi}_{S L}^{\kappa}=\left\{\gamma_{N}\right\}_{\Gamma} \circ \boldsymbol{\Psi}_{D L}^{\kappa} \quad, \quad \mathbf{C}_{\kappa}:=\left\{\gamma_{\mathbf{t}}\right\}_{\Gamma} \circ \boldsymbol{\Psi}_{D L}^{\kappa}=\left\{\gamma_{N}\right\}_{\Gamma} \circ \boldsymbol{\Psi}_{S L}^{\kappa} .
$$

The continuity of $\mathbf{S}_{\kappa}$ and $\mathbf{C}_{\kappa}$ is immediate from Theorem 5 , in conjunction with Lemma 3 and Theorem 1.

Corollary 2. The operators $\mathbf{S}_{\kappa}, \mathbf{C}_{\kappa}: \boldsymbol{H}_{\times}^{-\frac{1}{2}}\left(\operatorname{div}_{\Gamma}, \Gamma\right) \mapsto \boldsymbol{H}_{\times}^{-\frac{1}{2}}\left(\operatorname{div}_{\Gamma}, \Gamma\right)$ are continuous.

As auxiliary boundary integral operators, which supply building blocks for $\mathbf{S}_{\kappa}$ and $\mathbf{C}_{\kappa}$, we introduce the two single layer boundary integral operators

$$
V_{\kappa}:=\{\gamma\}_{\Gamma} \circ \Psi_{V}^{\kappa} \quad, \quad \mathbf{V}_{\kappa}:=\left\{\gamma_{\mathbf{t}}\right\}_{\Gamma} \circ \boldsymbol{\Psi}_{\mathbf{V}}^{\kappa} .
$$

By combining Lemma 4 with continuity properties of the traces, we obtain the following result

Corollary 3. The boundary integral operators $V_{\kappa}: H^{-\frac{1}{2}}(\Gamma) \mapsto H^{\frac{1}{2}}(\Gamma)$ and $\mathbf{V}_{\kappa}$ : $\boldsymbol{H}_{\times}^{-\frac{1}{2}}(\Gamma) \mapsto \boldsymbol{H}_{\times}^{\frac{1}{2}}(\Gamma)$ are continuous.

By inspecting the potential $\boldsymbol{\Psi}_{S L}^{\kappa}$, and recalling $\gamma_{\mathbf{t}} \circ \operatorname{grad}=\operatorname{curl}_{\Gamma} \circ \gamma$, it is clear that we can write

$$
\mathbf{S}_{\kappa}=\kappa \mathbf{V}_{\kappa}+\kappa^{-1} \operatorname{curl}_{\Gamma} \circ V_{\kappa} \circ \operatorname{div}_{\Gamma} .
$$

For the sake of implementation, more concrete boundary integral representations of the boundary integral operators are indispensable. It takes subtle theory to establish them, but here we only cite the result. A comprehensive treatment for second-order elliptic operators is given in [52, Section 7.2]. As variational formulations are our primary concern, expressions for the bilinear forms associated with $\mathbf{S}_{\kappa}$ and $\mathbf{C}_{\kappa}$ will be given: for tangential vectorfields $\boldsymbol{\mu}, \boldsymbol{\xi} \in \boldsymbol{L}^{\infty}(\Gamma)$ we obtain

$$
\begin{aligned}
\left\langle\mathbf{S}_{\kappa} \boldsymbol{\mu}, \boldsymbol{\xi}\right\rangle_{\boldsymbol{\tau}, \Gamma}= & -\kappa \int_{\Gamma} \int_{\Gamma} E_{\kappa}(\mathbf{x}-\mathbf{y}) \boldsymbol{\mu}(\mathbf{y}) \cdot \boldsymbol{\xi}(\mathbf{x}) \mathrm{d} S(\mathbf{y}, \mathbf{x})+ \\
& +\frac{1}{\kappa} \int_{\Gamma} \int_{\Gamma} E_{\kappa}(\mathbf{x}-\mathbf{y}) \operatorname{div}_{\Gamma} \boldsymbol{\mu}(\mathbf{y}) \operatorname{div}_{\Gamma} \boldsymbol{\xi}(\mathbf{x}) \mathrm{d} S(\mathbf{y}, \mathbf{x}), \\
\left\langle\mathbf{C}_{\kappa} \boldsymbol{\mu}, \boldsymbol{\xi}\right\rangle_{\boldsymbol{\tau}, \Gamma}= & -\int_{\Gamma} \int_{\Gamma} \operatorname{grad}_{\mathbf{x}} E_{\kappa}(\mathbf{x}-\mathbf{y}) \cdot(\boldsymbol{\mu}(\mathbf{y}) \times \boldsymbol{\xi}(\mathbf{x})) \mathrm{d} S(\mathbf{y}, \mathbf{x})
\end{aligned}
$$


The first integral arises from (31) through integration by parts. Its kernel $E_{\kappa}(\mathbf{x}-\mathbf{y})$ is weakly singular, because $E_{\kappa}(\mathbf{x}-\mathbf{y})=O\left(|\mathbf{x}-\mathbf{x}|^{-1}\right)$ for $\mathbf{y} \rightarrow \mathbf{x}$. Thus, the integral makes sense as an improper integral. The second integral has a strongly singular kernel behaving like $O\left(|\mathbf{x}-\mathbf{y}|^{-2}\right)$ for $\mathbf{y} \rightarrow \mathbf{x}$, and has to be read as a Cauchy principal value.

A fundamental tool for deriving boundary integral equations are jump relations describing the behavior of the potentials when crossing $\Gamma$. For the Maxwell single and double layer potential they closely resemble those for conventional single and double layer potentials for second order elliptic operators [52, Chapter 6]. For smooth domains these results are contained in [31, Thm. 6.11], [53, Thm. 5.5.1], and [57].

Theorem 7 (Jump relations). The interior and exterior Dirichlet- and Neumanntraces of the potentials $\boldsymbol{\Psi}_{S L}^{\kappa}$ and $\boldsymbol{\Psi}_{D L}^{\kappa}$ are well defined and, on $\boldsymbol{H}_{\times}^{-\frac{1}{2}}\left(\operatorname{div}_{\Gamma}, \Gamma\right)$, satisfy

$$
\left[\gamma_{\mathbf{t}}\right]_{\Gamma} \circ \boldsymbol{\Psi}_{S L}^{\kappa}=\left[\gamma_{N}\right]_{\Gamma} \circ \boldsymbol{\Psi}_{D L}^{\kappa}=0, \quad\left[\gamma_{N}\right]_{\Gamma} \circ \boldsymbol{\Psi}_{S L}^{\kappa}=\left[\gamma_{\mathbf{t}}\right]_{\Gamma} \circ \boldsymbol{\Psi}_{D L}^{\kappa}=-\mathrm{Id} .
$$

Proof. The jump condition for the Dirichlet trace of the single layer potential is immediate from its regularity asserted in Lemma 4. By (30) we get the continuity of the Neumann trace $\boldsymbol{\Psi}_{D L}^{\kappa}$. Then, the jump of the Neumann trace of $\boldsymbol{\Psi}_{S L}^{\kappa}$ can be determined from (26). Finally, by (30), this also settles the contention for the Dirichlet trace of the double layer potential.

Now, with the jump relations in mind, let us apply the exterior and interior trace operators to the representation formulae of Theorem 6:

$$
\begin{array}{ll}
\gamma_{\mathbf{t}}^{-} \mathbf{u}=\frac{1}{2} \gamma_{\mathbf{t}}^{-} \mathbf{u}+\mathbf{C}_{\kappa}\left(\gamma_{\mathbf{t}}^{-} \mathbf{u}\right)+\mathbf{S}_{\kappa}\left(\gamma_{N}^{-} \mathbf{u}\right), & \gamma_{\mathbf{t}}^{+} \mathbf{u}=\frac{1}{2} \gamma_{\mathbf{t}}^{+} \mathbf{u}-\mathbf{C}_{\kappa}\left(\gamma_{\mathbf{t}}^{+} \mathbf{u}\right)-\mathbf{S}_{\kappa}\left(\gamma_{N}^{+} \mathbf{u}\right), \\
\gamma_{N}^{-} \mathbf{u}=\mathbf{S}_{\kappa}\left(\gamma_{\mathbf{t}}^{-} \mathbf{u}\right)+\frac{1}{2} \gamma_{N}^{-} \mathbf{u}+\mathbf{C}_{\kappa}\left(\gamma_{N}^{-} \mathbf{u}\right), & \gamma_{N}^{+} \mathbf{u}=-\mathbf{S}_{\kappa}\left(\gamma_{\mathbf{t}}^{+} \mathbf{u}\right)+\frac{1}{2} \gamma_{N}^{+} \mathbf{u}-\mathbf{C}_{\kappa}\left(\gamma_{N}^{+} \mathbf{u}\right) .
\end{array}
$$

A concise way to write these formulae relies on the Calderon projectors, $c f$. [21, Section 3.3], [37, Formula (29)], and [53, Sect. 5.5],

$$
\mathbb{P}_{\kappa}^{-}:=\left(\begin{array}{cc}
\frac{1}{2} \operatorname{Id}+\mathbf{C}_{\kappa} & \mathbf{S}_{\kappa} \\
\mathbf{S}_{\kappa} & \frac{1}{2} \operatorname{Id}+\mathbf{C}_{\kappa}
\end{array}\right), \quad \mathbb{P}_{\kappa}^{+}:=\left(\begin{array}{cc}
\frac{1}{2} \operatorname{Id}-\mathbf{C}_{\kappa} & -\mathbf{S}_{\kappa} \\
-\mathbf{S}_{\kappa} & \frac{1}{2} \operatorname{Id}-\mathbf{C}_{\kappa}
\end{array}\right) .
$$

By Theorem 6 the operators $\mathbb{P}_{\kappa}^{-}, \mathbb{P}_{\kappa}^{+}: \boldsymbol{H}_{\times}^{-\frac{1}{2}}\left(\operatorname{div}_{\Gamma}, \Gamma\right)^{2} \mapsto \boldsymbol{H}_{\times}^{-\frac{1}{2}}\left(\operatorname{div}_{\Gamma}, \Gamma\right)^{2}$ are projectors, that is,

$$
\mathbb{P}_{\kappa}^{-} \circ \mathbb{P}_{\kappa}^{-}=\mathbb{P}_{\kappa}^{-}, \quad \mathbb{P}_{\kappa}^{+} \circ \mathbb{P}_{\kappa}^{+}=\mathbb{P}_{\kappa}^{+} .
$$

Also note that $\mathbb{P}_{\kappa}^{-}+\mathbb{P}_{\kappa}^{+}=\mathrm{Id}$ and that the range of $\mathbb{P}_{\kappa}^{+}$coincides with the kernel of $\mathbb{P}_{\kappa}^{-}$and vice versa. The next result promotes Calderon projectors to a pivotal role in the derivation of boundary integral equations, $c f$. [61, Thm. 3.7].

Theorem 8. The pair of functions $(\boldsymbol{\zeta}, \boldsymbol{\mu}) \in \boldsymbol{H}_{\times}^{-\frac{1}{2}}\left(\operatorname{div}_{\Gamma}, \Gamma\right) \times \boldsymbol{H}_{\times}^{-\frac{1}{2}}\left(\operatorname{div}_{\Gamma}, \Gamma\right)$ are suitable interior or exterior Maxwell Cauchy data, if and only if they lie in the kernel of $\mathbb{P}_{\kappa}^{+}$or $\mathbb{P}_{\kappa}^{-}$, respectively. 
For the subsequent analysis it is convenient to examine the operator

$$
\mathbb{A}_{\kappa}:=\left(\begin{array}{cc}
\mathbf{C}_{\kappa} & \mathbf{S}_{\kappa} \\
\mathbf{S}_{\kappa} & \mathbf{C}_{\kappa}
\end{array}\right): \boldsymbol{H}_{\times}^{-\frac{1}{2}}\left(\operatorname{div}_{\Gamma}, \Gamma\right)^{2} \mapsto \boldsymbol{H}_{\times}^{-\frac{1}{2}}\left(\operatorname{div}_{\Gamma}, \Gamma\right)^{2}
$$

It is linked with the Calderon projectors by $\mathbb{P}_{\kappa}^{-}=\frac{1}{2} \operatorname{Id}+\mathbb{A}_{\kappa}, \mathbb{P}_{\kappa}^{+}=\frac{1}{2} \operatorname{Id}-\mathbb{A}_{\kappa}$.

The operators $\mathbf{C}_{\kappa}$ enjoy a hidden symmetry, made precise in the next lemma, see $[21$, Thm. 3.9].

Lemma 6. We have $\left\langle\mathbf{C}_{\kappa} \boldsymbol{\zeta}, \boldsymbol{\mu}\right\rangle_{\boldsymbol{\tau}, \Gamma}=\left\langle\mathbf{C}_{\kappa} \boldsymbol{\mu}, \boldsymbol{\zeta}\right\rangle_{\boldsymbol{\tau}, \Gamma}$ for all $\boldsymbol{\zeta}, \boldsymbol{\mu} \in \boldsymbol{H}_{\times}^{-\frac{1}{2}}\left(\operatorname{div}_{\Gamma}, \Gamma\right)$.

\section{Compactness and Coercivity}

The ultimate goal is to establish the coercivity of bilinear forms occuring in weak formulations of boundary integral equations. To achieve this we need to identify compact perturbations, $c f$. Lemma 3.2 of [47] and the proof of Thm. 3.12 in [21].

Lemma 7. The integral operators $\delta V_{\kappa}:=V_{\kappa}-V_{0}: H^{-\frac{1}{2}}(\Gamma) \mapsto H^{\frac{1}{2}}(\Gamma)$ and $\delta \mathbf{V}_{\kappa}:=\mathbf{V}_{\kappa}-\mathbf{V}_{0}: \boldsymbol{H}_{\times}^{-\frac{1}{2}}(\Gamma) \mapsto \boldsymbol{H}_{\times}^{\frac{1}{2}}(\Gamma)$ are compact.

Slightly abusing notation, we define

$$
\mathbf{S}_{0}:=\kappa \mathbf{V}_{0}+\kappa^{-1} \operatorname{curl}_{\Gamma} \circ V_{0} \circ \operatorname{div}_{\Gamma}
$$

¿From Lemma 7 and (31) we find that switching from $\mathbf{S}_{\kappa}$ to $\mathbf{S}_{0}$ amounts to a compact perturbation.

Corollary 4. The operator $\mathbf{S}_{\kappa}-\mathbf{S}_{0}: \boldsymbol{H}_{\times}^{-\frac{1}{2}}\left(\operatorname{div}_{\Gamma}, \Gamma\right) \mapsto \boldsymbol{H}_{\times}^{-\frac{1}{2}}\left(\operatorname{div}_{\Gamma}, \Gamma\right)$ is compact.

The significance of this can be appreciated in light of the following result, $c f$. Thm. 3 in [36, Vol. IV, Ch. XI, $\S 2$ ], and Thm. 6.2 in [46].

Lemma 8 (Ellipticity of single layer potentials). The operators $V_{0}$ and $\mathbf{V}_{0}$ are continuous, selfadjoint with respect to the bilinear pairings $\langle\cdot, \cdot\rangle_{\frac{1}{2}, \Gamma}$ and $\langle\cdot, \cdot\rangle_{\boldsymbol{\tau}, \Gamma}$, respectively, and satisfy

$$
\begin{array}{ll}
\left\langle\mu, V_{0} \bar{\mu}\right\rangle_{\frac{1}{2}, \Gamma} \geq C\|\mu\|_{H^{-\frac{1}{2}}(\Gamma)}^{2} & \forall \mu \in H^{-\frac{1}{2}}(\Gamma), \\
\left\langle\boldsymbol{\mu}, \mathbf{V}_{0} \overline{\boldsymbol{\mu}}\right\rangle_{\boldsymbol{\tau}, \Gamma} \geq C\|\boldsymbol{\mu}\|_{\boldsymbol{H}_{\times}^{-\frac{1}{2}}(\Gamma)}^{2} & \forall \boldsymbol{\mu} \in \boldsymbol{H}_{\times}^{-\frac{1}{2}}\left(\operatorname{div}_{\Gamma} 0, \Gamma\right) .
\end{array}
$$

with constants $C>0$ only depending on $\Gamma$.

Again, it proves highly instructive to remember facts about boundary integral operators related to the Helmholtz equation: Table 3 lists similarities and differences of the situations faced in the case of the Helmholtz equation and Maxwell's equations, respectively. The lack of ellipticity of the off-diagonal operators in $\mathbb{A}_{\kappa}$ 
Table 3. Comparison of analytical aspects of the acoustic and electromagnetic boundary integral operators, supplementing Table 1 . The symbols $K_{\kappa}$ and $D_{\kappa}$ denote the double layer integral operator, and the hypersingular integral operator for the Helmholtz operator $-\Delta-\kappa^{2} \mathrm{Id}$, respectively. Details can be found in [52, Ch. 9].

\begin{tabular}{c|c} 
Helmholtz equation & Maxwell equations \\
\hline$-\Delta p-\kappa^{2} p=0$ & $\operatorname{curl} \operatorname{curl} \mathbf{e}-\kappa^{2} \mathbf{e}=0$
\end{tabular}

Boundary integral operators:

$$
\begin{aligned}
& \text { Dirichlet trace } \gamma p \in H^{\frac{1}{2}}(\Gamma) \quad \text { Dirichlet trace } \gamma_{\mathbf{t}} \mathbf{e} \in \boldsymbol{H}_{\times}^{-\frac{1}{2}}\left(\operatorname{div}_{\Gamma}, \Gamma\right) \\
& \text { Neumann trace } \frac{1}{\kappa} \gamma_{\mathbf{n}}(\operatorname{grad} p) \in H^{-\frac{1}{2}}(\Gamma) \quad \text { Neumann trace } \frac{1}{\kappa} \gamma_{\mathbf{t}}(\mathbf{c u r l} \mathbf{e}) \in \boldsymbol{H}_{\times}^{-\frac{1}{2}}\left(\operatorname{div}_{\Gamma}, \Gamma\right) \\
& \mathbb{A}_{\kappa}=\left(\begin{array}{cc}
K_{\kappa} & V_{\kappa} \\
D_{\kappa} & \tilde{K}_{\kappa}
\end{array}\right) \\
& \mathbb{A}_{\kappa}=\left(\begin{array}{ll}
\mathbf{C}_{\kappa} & \mathbf{S}_{\kappa} \\
\mathbf{S}_{\kappa} & \mathbf{C}_{\kappa}
\end{array}\right)
\end{aligned}
$$

The issue of coercivity

$$
\mathbb{A}_{\kappa}=\left(\begin{array}{cc}
K_{\kappa} & V_{0} \\
D_{0} & K_{\kappa}^{*}
\end{array}\right)+\text { compact pert. }
$$

Ellipticity on trace spaces:

$$
\begin{aligned}
\left\langle\varphi, V_{0} \bar{\varphi}\right\rangle_{\frac{1}{2}, \Gamma} \geq C\|\varphi\|_{H^{-\frac{1}{2}}(\Gamma)}^{2}, \\
\left\langle D_{0} \varphi, \bar{\varphi}\right\rangle_{\frac{1}{2}, \Gamma} \geq C\|\varphi\|_{H^{\frac{1}{2}}(\Gamma) / \mathbb{C}}^{2}
\end{aligned}
$$

$$
\mathbb{A}_{\kappa}=\left(\begin{array}{ll}
\mathbf{C}_{\kappa} & \mathbf{S}_{0} \\
\mathbf{S}_{0} & \mathbf{C}_{\kappa}
\end{array}\right)+\text { compact pert }
$$

No ellipticity, because $\mathbf{S}_{0}$ indefinite:

$$
\mathbf{S}_{0}=\mathbf{V}_{0}+\frac{1}{\kappa} \operatorname{curl}_{\Gamma} \circ V_{0} \circ \operatorname{div}_{\Gamma} .
$$

Yet, individual terms are (semi)-definite.

has the same roots as the absence of a direct compact embedding in the case of the Maxwell source problem, $c f$. Section 3.

The roots of the difficulties being the same as for the Maxwell source problem, the same ideas should provide remedies: we have to employ stable splittings that target the trace space $\boldsymbol{H}_{\times}^{-\frac{1}{2}}\left(\operatorname{div}_{\Gamma}, \Gamma\right)$ and decompose it into the kernel of $\operatorname{div}_{\Gamma}$ and a suitable more regular complement. The decomposition (21) introduced in Sect. 3 meets all the requirements and will be used below.

Please recall the discussion in Sect. 3 of the coercivity of the bilinear form associated with the Maxwell source problem. The same considerations will now be applied to the bilinear form spawned by the boundary integral operator $\mathbf{S}_{\kappa}$ through $(\boldsymbol{\xi}, \boldsymbol{\mu}) \mapsto\left\langle\mathbf{S}_{\kappa} \boldsymbol{\mu}, \boldsymbol{\xi}\right\rangle_{\boldsymbol{\tau}, \Gamma}$. To begin with, the "lower order" term $\mathbf{V}_{\kappa}$ in the operator $\mathbf{S}_{\kappa}, c f$. Formula (31), becomes compact on the "regular component" $\mathcal{X}(\Gamma)$ of the decomposition (21). 
Lemma 9. The bilinearforms $\left\langle\mathbf{V}_{\kappa} \cdot, \cdot\right\rangle_{\boldsymbol{\tau}, \Gamma}: \mathcal{X}(\Gamma) \times \boldsymbol{H}_{\times}^{-\frac{1}{2}}(\Gamma) \mapsto \mathbb{C}$ and $\left\langle\mathbf{V}_{\kappa} \cdot, \cdot\right\rangle_{\boldsymbol{\tau}, \Gamma}$ : $\boldsymbol{H}_{\times}^{-\frac{1}{2}}(\Gamma) \times \mathcal{X}(\Gamma) \mapsto \mathbb{C}$ are compact.

Proof. Since $\mathbf{V}_{\kappa}: \boldsymbol{H}_{\times}^{-\frac{1}{2}}(\Gamma) \mapsto \boldsymbol{H}_{\times}^{\frac{1}{2}}(\Gamma)$ is continuous according to Theorem 3, the compact embedding $\mathcal{X}(\Gamma) \hookrightarrow \boldsymbol{H}_{\times}^{-\frac{1}{2}}(\Gamma)$ (Corollary 1) gives the result.

We can even establish a generalized Gårding inequality for $\mathbf{S}_{0}$ on Lipschitz boundaries: looking at the formula (31) and, in particular, the bilinear form

$$
\left\langle\mathbf{S}_{0} \boldsymbol{\mu}, \boldsymbol{\xi}\right\rangle_{\boldsymbol{\tau}, \Gamma}=\frac{1}{\kappa}\left\langle\operatorname{div}_{\Gamma} \boldsymbol{\mu}, V_{0} \operatorname{div}_{\Gamma} \boldsymbol{\mu}\right\rangle_{\frac{1}{2}, \Gamma}-\kappa\left\langle\boldsymbol{\mu}, \mathbf{V}_{0} \boldsymbol{\xi}\right\rangle_{\boldsymbol{\tau}, \Gamma},
$$

we realize a striking similarity to the bilinear form of the Maxwell source problem (15). Thus, it is natural to employ the splitting idea of Sect. 3 based on (21) and the isomorphism

$$
\mathrm{X}_{\Gamma}=\mathrm{R}^{\Gamma}-\mathrm{Z}^{\Gamma}: \boldsymbol{H}_{\times}^{-\frac{1}{2}}\left(\operatorname{div}_{\Gamma}, \Gamma\right) \mapsto \boldsymbol{H}_{\times}^{-\frac{1}{2}}\left(\operatorname{div}_{\Gamma}, \Gamma\right) .
$$

Lemma 10 (Generalized Gårding inequality for $\mathbf{S}_{\kappa}$ ). There is a compact bilinear form $c_{\Gamma}: \boldsymbol{H}_{\times}^{-\frac{1}{2}}\left(\operatorname{div}_{\Gamma}, \Gamma\right) \times \boldsymbol{H}_{\times}^{-\frac{1}{2}}\left(\operatorname{div}_{\Gamma}, \Gamma\right) \mapsto \mathbb{C}$ and a constant $C_{G}>0$ such that

$$
\left|\left(\mathbf{S}_{\kappa} \boldsymbol{\mu}, \mathrm{X}_{\Gamma} \overline{\boldsymbol{\mu}}\right)_{\boldsymbol{\tau}}+c_{\Gamma}(\boldsymbol{\mu}, \overline{\boldsymbol{\mu}})\right| \geq C_{G}\|\boldsymbol{\mu}\|_{\boldsymbol{H}_{\times}^{-\frac{1}{2}}\left(\operatorname{div}_{\Gamma}, \Gamma\right)}^{2} \quad \forall \boldsymbol{\mu} \in \boldsymbol{H}_{\times}^{-\frac{1}{2}}\left(\operatorname{div}_{\Gamma}, \Gamma\right) .
$$

Proof. We set

$$
c_{\Gamma}(\boldsymbol{\mu}, \boldsymbol{\xi}):=-\left\langle\mathbf{V}_{\kappa} \mathrm{R}^{\Gamma} \boldsymbol{\mu}, \mathrm{R}^{\Gamma} \boldsymbol{\xi}\right\rangle_{\boldsymbol{\tau}, \Gamma}+\left\langle\mathbf{V}_{\kappa} \mathrm{R}^{\Gamma} \boldsymbol{\mu}, \mathrm{Z}^{\Gamma} \boldsymbol{\xi}\right\rangle_{\boldsymbol{\tau}, \Gamma}-\left\langle\mathbf{V}_{\kappa} \mathrm{Z}^{\Gamma} \boldsymbol{\mu}, \mathrm{R}^{\Gamma} \boldsymbol{\xi}\right\rangle_{\boldsymbol{\tau}, \Gamma},
$$

which is compact by Lemma 9. Noting that

$\left\langle\mathbf{S}_{0} \boldsymbol{\mu}, \mathbf{X}_{\Gamma} \overline{\boldsymbol{\xi}}\right\rangle_{\boldsymbol{\tau}, \Gamma}=\frac{1}{\kappa}\left\langle V_{0} \operatorname{div}_{\Gamma} \mathrm{R}^{\Gamma} \boldsymbol{\mu}, \operatorname{div}_{\Gamma} \mathrm{R}^{\Gamma} \overline{\boldsymbol{\xi}}\right\rangle_{0 ; \Gamma}+\kappa\left\langle\mathrm{Z}^{\Gamma} \boldsymbol{\mu}, \mathbf{V}_{0} \mathrm{Z}^{\Gamma} \overline{\boldsymbol{\xi}}\right\rangle_{\boldsymbol{\tau}, \Gamma}-c_{\Gamma}(\boldsymbol{\mu}, \overline{\boldsymbol{\xi}})$, we invoke Lemma 8 and the stability of the decomposition to finish the proof.

In the case of smooth domains this result is sufficient to obtain coercivity of $\mathbb{A}_{\kappa}$, because for smooth boundaries the singularity of the kernel of $\mathbf{C}_{\kappa}$ partly cancels. This is a well-known effect in the case of double layer potentials for second order elliptic operators. For $\mathbf{C}_{\kappa}$ the observation was made by Nédélec [53, Section 5.5].

Lemma 11. If $\Gamma$ is smooth, that is, of class $C^{\infty}$, then $\mathbf{C}_{\kappa}$ is continuous as an operator $\mathbf{C}_{\kappa}: \boldsymbol{H}_{\mathbf{t}}^{s}(\Gamma) \mapsto \boldsymbol{H}_{\mathbf{t}}^{s+1}(\Gamma)$ and $\mathbf{C}_{\kappa}: \boldsymbol{T} \boldsymbol{H}^{s-\frac{1}{2}}\left(\operatorname{div}_{\Gamma} ; \Gamma\right) \mapsto \boldsymbol{T} \boldsymbol{H}^{s+\frac{1}{2}}\left(\operatorname{div}_{\Gamma} ; \Gamma\right)$ for all $s \in \mathbb{R}$.

Proof. The first part of the proof boils down to manipulations of (33) using the product rule for curl $_{\mathbf{x}}$ and the identity $(\mathbf{b} \times \mathbf{c}) \times \mathbf{a}=\mathbf{c}(\mathbf{a} \cdot \mathbf{b})-\mathbf{b}(\mathbf{a} \cdot \mathbf{c})$. For $\boldsymbol{\mu}, \boldsymbol{\xi} \in \mathbf{L}^{\infty}(\Gamma) \cap \boldsymbol{T} \boldsymbol{H}\left(\operatorname{div}_{\Gamma} ; \Gamma\right)$ we end up with 


$$
\begin{aligned}
& \left\langle\mathbf{C}_{\kappa} \boldsymbol{\mu}, \boldsymbol{\xi}\right\rangle_{\boldsymbol{\tau}, \Gamma}= \\
= & -\int_{\Gamma} \int_{\Gamma}\left(\left(\boldsymbol{\mu}(\mathbf{y}) \times \operatorname{grad}_{\mathbf{x}} E_{\kappa}(\mathbf{x}-\mathbf{y})\right) \times \mathbf{n}(\mathbf{x})\right) \cdot(\boldsymbol{\xi}(\mathbf{x}) \times \mathbf{n}(\mathbf{x})) \mathrm{d} S(\mathbf{y}, \mathbf{x}) \\
= & -\int_{\Gamma} \int_{\Gamma} \boldsymbol{\mu}(\mathbf{y})\left(\operatorname{grad}_{\mathbf{x}} E_{\kappa}(\mathbf{x}-\mathbf{y}) \cdot \mathbf{n}(\mathbf{x})\right) \cdot(\boldsymbol{\xi}(\mathbf{x}) \times \mathbf{n}(\mathbf{x})) \mathrm{d} S(\mathbf{y}, \mathbf{x})+ \\
& +\int_{\Gamma} \int_{\Gamma} \operatorname{grad}_{\mathbf{x}} E_{\kappa}(\mathbf{x}-\mathbf{y})(\boldsymbol{\mu}(\mathbf{y}) \cdot(\mathbf{n}(\mathbf{x})-\mathbf{n}(\mathbf{y}))) \cdot(\boldsymbol{\xi}(\mathbf{x}) \times \mathbf{n}(\mathbf{x})) \mathrm{d} S(\mathbf{y}, \mathbf{x}) .
\end{aligned}
$$

According to [25, Section 6.4] we have $|\mathbf{n}(\mathbf{x})-\mathbf{n}(\mathbf{y})|=O(|\mathbf{x}-\mathbf{y}|)$ for smooth surfaces. Thus, a closer scrutiny of the formulae shows that

$\operatorname{grad}_{\mathbf{x}} E_{\kappa}(\mathbf{x}-\mathbf{y}) \cdot \mathbf{n}(\mathbf{x}) \simeq \operatorname{grad}_{\mathbf{x}} E_{\kappa}(\mathbf{x}-\mathbf{y})(\mathbf{n}(\mathbf{x})-\mathbf{n}(\mathbf{y}))^{T} \simeq O\left(|\mathbf{x}-\mathbf{y}|^{-1}\right)$,

for $\mathbf{x} \rightarrow \mathbf{y}$. Both kernels are weakly singular, as is the kernel of $\mathbf{S}_{\kappa}$. So the theory of pseudo-differential operators [25, Ch. 4.4] shows that $\mathbf{C}_{\kappa}$ is continuous as an operator from $\boldsymbol{H}_{\mathbf{t}}^{s}(\Gamma) \mapsto \boldsymbol{H}_{\mathbf{t}}^{s+1}(\Gamma), s \in \mathbb{R}$ (Note that on a smooth boundary the infinite scale of Sobolev spaces is available).

Next, pick a smooth tangential vector-field $\boldsymbol{\mu}$, use Lemma 5 and apply simple manipulations based on vector identities

$$
\begin{aligned}
\operatorname{div}_{\Gamma} \mathbf{C}_{\kappa}(\boldsymbol{\mu})(\mathbf{x})=\frac{1}{\kappa} \text { curl curl } \int_{\Gamma} E_{\kappa}(\mathbf{x}-\mathbf{y}) \boldsymbol{\mu}(\mathbf{y}) \mathrm{d} S \cdot \mathbf{n}(\mathbf{x}) \\
=\frac{1}{\kappa} \int_{\Gamma} \frac{\partial}{\partial \mathbf{n}(\mathbf{x})} E_{\kappa}(\mathbf{x}-\mathbf{y}) \operatorname{div}_{\Gamma} \boldsymbol{\mu}(\mathbf{y}) \mathrm{d} S+\kappa \int_{\Gamma} E_{\kappa}(\mathbf{x}-\mathbf{y}) \boldsymbol{\mu}(\mathbf{y}) \mathrm{d} S \cdot \mathbf{n}(\mathbf{x}) .
\end{aligned}
$$

By density, we conclude that $\operatorname{div}_{\Gamma} \circ \mathbf{C}_{\kappa}: \boldsymbol{T} \boldsymbol{H}^{s-\frac{1}{2}}\left(\operatorname{div}_{\Gamma} ; \Gamma\right) \mapsto H^{s+\frac{1}{2}}(\Gamma)$ is continuous. This can be combined with the previous results and confirms the second assertion of the theorem.

The crucial message sent by this lemma and Lemma 7 is that on smooth boundaries the operator $\mathbb{A}_{\kappa}: \boldsymbol{H}_{\times}^{-\frac{1}{2}}\left(\operatorname{div}_{\Gamma}, \Gamma\right)^{2} \mapsto \boldsymbol{H}_{\times}^{-\frac{1}{2}}\left(\operatorname{div}_{\Gamma}, \Gamma\right)^{2}$ can be converted into

$$
\mathbb{A}_{\kappa} \simeq\left(\begin{array}{cc}
0 & \mathbf{S}_{0} \\
\mathbf{S}_{0} & 0
\end{array}\right) .
$$

by dropping "compact perturbations"3. In other words, Dirichlet and Neumann traces are coupled by compact terms only. On smooth boundaries we merely have to examine $\mathbf{S}_{\kappa}$, if we are interested in coercivity.

Unfortunately, the coupling terms $\mathbf{C}_{\kappa}$ in $\mathbb{A}_{\kappa}$ cannot be discarded in the case of non-smooth boundaries for want of a result like Lemma 11: in general, we have to

\footnotetext{
${ }^{3}$ Here and below we use the symbol $\simeq$ to express equality of operators and bilinear forms up to addition of compact terms
} 
deal with the two different traces $\gamma_{\mathbf{t}} \mathbf{e}$ and $\gamma_{N} \mathbf{e}$ together. This is a completely new aspect of boundary integral operators that we have not encountered in the case of the Maxwell source problem. Thus, splitting alone is not enough, but has to be accompanied by an appropriate grouping of the components. This is where the "physical meaning" of the splitting (21) that we discussed in Table 2 offers an important hint: it suggests that we distinguish between trace components of electric and magnetic nature. The ultimate justification for this idea is the profound result that it is merely compact terms, by which electric and magnetic components are coupled in the boundary integral operator $\mathbb{A}_{\kappa}$. The next lemma rigorously expresses this insight, $c f$. Prop. 3.13 in [21].

Lemma 12. The bilinear form $\left\langle\mathbf{C}_{\kappa} \cdot, \cdot\right\rangle_{\boldsymbol{\tau}, \Gamma}$ is compact both on $\mathcal{N}(\Gamma) \times \mathcal{N}(\Gamma)$ and $\mathcal{X}(\Gamma) \times \mathcal{X}(\Gamma)$.

Proof. We restrict ourselves to the proof of the second assertion. We choose some $\boldsymbol{\zeta}, \boldsymbol{\mu} \in \mathcal{X}(\Gamma)$ and recall the definition of $\mathbf{C}_{\kappa}$ along with the jump relations. It is important to note that, by virtue of the definition of $\mathcal{X}(\Gamma), \boldsymbol{\mu}$ can be extended by $\mathbf{v}:=\mathrm{J}\left(\operatorname{div}_{\Gamma} \boldsymbol{\mu}\right) \in \boldsymbol{H}^{1}\left(\Omega_{s}\right), \mathrm{J}$ defined in the proof of Lemma 2 , such that $\gamma_{\mathbf{t}} \mathbf{v}=\boldsymbol{\mu}$ and $\|\mathbf{v}\|_{\boldsymbol{H}^{1}\left(\Omega_{s}\right)} \leq C\|\boldsymbol{\mu}\|_{\boldsymbol{H}_{\times}^{-\frac{1}{2}}\left(\operatorname{div}_{\Gamma}, \Gamma\right)}$. Also, exploiting $\operatorname{div} \mathbf{v}=0$, we get

$$
\left\langle\mathbf{C}_{\kappa} \boldsymbol{\zeta}, \boldsymbol{\mu}\right\rangle_{\boldsymbol{\tau}, \Gamma}=\left\langle\gamma_{N}^{-} \boldsymbol{\Psi}_{\mathbf{V}}^{\kappa}(\boldsymbol{\zeta}), \boldsymbol{\mu}\right\rangle_{\boldsymbol{\tau}, \Gamma}-\frac{1}{2}\langle\boldsymbol{\zeta}, \boldsymbol{\mu}\rangle_{\boldsymbol{\tau}, \Gamma}
$$

Using the identity $\operatorname{curl} \operatorname{curl} \boldsymbol{\Psi}_{\mathbf{V}}^{\kappa}(\boldsymbol{\zeta})=\operatorname{grad} \Psi_{V}^{\kappa}\left(\operatorname{div}_{\Gamma} \boldsymbol{\zeta}\right)+\kappa^{2} \boldsymbol{\Psi}_{\mathbf{V}}^{\kappa}(\boldsymbol{\zeta})$ and the integration by parts (5), we obtain:

$$
\begin{aligned}
\left\langle\gamma_{N}^{-} \boldsymbol{\Psi}_{\mathbf{V}}^{\kappa}(\boldsymbol{\zeta}), \boldsymbol{\mu}\right\rangle_{\boldsymbol{\tau}, \Gamma}= & -\int_{\Omega_{s}} \operatorname{curl} \boldsymbol{\Psi}_{\mathbf{V}}^{\kappa} \cdot \operatorname{curl} \mathbf{v}+\kappa^{2} \boldsymbol{\Psi}_{\mathbf{V}}^{\kappa}(\boldsymbol{\zeta}) \cdot \mathbf{v} \mathrm{d} \mathbf{x}+ \\
& +\left\langle\gamma^{-} \Psi_{V}^{\kappa}\left(\operatorname{div}_{\Gamma} \mu\right), \gamma_{\mathbf{n}}^{-} \mathbf{v}\right\rangle_{\frac{1}{2}, \Gamma}
\end{aligned}
$$

This means that

$$
\begin{aligned}
\left|\left(\mathbf{C}_{\kappa}(\boldsymbol{\zeta}), \boldsymbol{\mu}\right)_{\boldsymbol{\tau}}\right| \leq & \left|\boldsymbol{\Psi}_{\mathbf{V}}^{\kappa}(\boldsymbol{\zeta})\right|_{\boldsymbol{H}^{1}\left(\Omega_{s}\right)}\|\operatorname{curl} \mathbf{v}\|_{\boldsymbol{L}^{2}\left(\Omega_{s}\right)}+\kappa^{2}\left\|\boldsymbol{\Psi}_{\mathbf{V}}^{\kappa}(\boldsymbol{\zeta})\right\|_{\boldsymbol{L}^{2}\left(\Omega_{s}\right)}\|\mathbf{v}\|_{\boldsymbol{L}^{2}\left(\Omega_{s}\right)} \\
& +\left\|V_{\kappa}\left(\operatorname{div}_{\Gamma} \boldsymbol{\zeta}\right)\right\|_{L^{2}(\Gamma)}\left\|\gamma_{\mathbf{n}}^{-} \mathbf{v}\right\|_{L^{2}(\Gamma)}+\|\boldsymbol{\zeta}\|_{\boldsymbol{L}_{\mathbf{t}}^{2}(\Gamma)}\left\|\gamma_{\mathbf{t}}^{-} \mathbf{v}\right\|_{\boldsymbol{L}_{\mathbf{t}}^{2}(\Gamma)} \\
\leq & C\left(\|\boldsymbol{\zeta}\|_{\boldsymbol{H}_{\times}^{-\frac{1}{2}}(\Gamma)}+\left\|V_{\kappa}\left(\operatorname{div}_{\Gamma} \boldsymbol{\zeta}\right)\right\|_{L^{2}(\Gamma)}+\|\boldsymbol{\zeta}\|_{\boldsymbol{L}_{\mathbf{t}}^{2}(\Gamma)}\right)\|\mathbf{v}\|_{\boldsymbol{H}^{1}\left(\Omega_{s}\right)}
\end{aligned}
$$

with some $C=C\left(\Omega_{s}\right)>0$. It goes without saying that the operator $V_{\kappa}$ : $H^{-\frac{1}{2}}(\Gamma) \mapsto L^{2}(\Gamma)$ is compact. Then, the compact embedding of $\mathcal{X}(\Gamma)$ in $\boldsymbol{L}_{\mathbf{t}}^{2}(\Gamma)$ according to Corollary 1 finishes the proof.

To understand the meaning of these results, we consider the combined boundary integral operator $\mathbb{A}_{\kappa}: \boldsymbol{H}_{\times}^{-\frac{1}{2}}\left(\operatorname{div}_{\Gamma}, \Gamma\right)^{2} \mapsto \boldsymbol{H}_{\times}^{-\frac{1}{2}}\left(\operatorname{div}_{\Gamma}, \Gamma\right)^{2}$ with respect to the splitting (21). As usual, we adopt a variational perspective and study the bilinear form asscociated with $\mathbb{A}_{\kappa}$. It will be based on the following anti-symmetric pairing on the product space $\boldsymbol{H}_{\times}^{-\frac{1}{2}}\left(\operatorname{div}_{\Gamma}, \Gamma\right) \times \boldsymbol{H}_{\times}^{-\frac{1}{2}}\left(\operatorname{div}_{\Gamma}, \Gamma\right)$, 


$$
\left\langle\left(\begin{array}{c}
\boldsymbol{\zeta} \\
\boldsymbol{\mu}
\end{array}\right),\left(\begin{array}{c}
\boldsymbol{\xi} \\
\boldsymbol{\lambda}
\end{array}\right)\right\rangle_{\boldsymbol{\tau} \times \boldsymbol{\tau}}:=\langle\boldsymbol{\zeta}, \boldsymbol{\lambda}\rangle_{\boldsymbol{\tau}, \Gamma}+\langle\boldsymbol{\mu}, \boldsymbol{\xi}\rangle_{\boldsymbol{\tau}, \Gamma}
$$

Now, pick $\boldsymbol{\zeta}, \boldsymbol{\mu}, \boldsymbol{\xi}, \boldsymbol{\lambda} \in \boldsymbol{H}_{\times}^{-\frac{1}{2}}\left(\operatorname{div}_{\Gamma}, \Gamma\right)$ and use superscripts $\perp$ and 0 to tag their components in $\mathcal{X}(\Gamma)$ and $\mathcal{N}(\Gamma)$, respectively.

$$
\begin{aligned}
\left\langle\mathbb{A}_{\kappa}\left(\begin{array}{c}
\boldsymbol{\zeta} \\
\boldsymbol{\mu}
\end{array}\right),\left(\begin{array}{c}
\boldsymbol{\xi} \\
\boldsymbol{\lambda}
\end{array}\right)\right\rangle_{\boldsymbol{\tau} \times \boldsymbol{\tau}}=\left\langle\mathbb{A}_{\kappa}\left(\begin{array}{c}
\boldsymbol{\zeta}^{\perp} \\
\boldsymbol{\mu}^{0}
\end{array}\right),\left(\begin{array}{c}
\boldsymbol{\xi}^{\perp} \\
\boldsymbol{\lambda}^{0}
\end{array}\right)\right\rangle_{\boldsymbol{\tau} \times \boldsymbol{\tau}}+\left\langle\mathbb{A}_{\kappa}\left(\begin{array}{c}
\boldsymbol{\zeta}^{0} \\
\boldsymbol{\mu}^{\perp}
\end{array}\right),\left(\begin{array}{c}
\boldsymbol{\xi}^{0} \\
\boldsymbol{\lambda}^{\perp}
\end{array}\right)\right\rangle_{\boldsymbol{\tau} \times \boldsymbol{\tau}}+ \\
+\left\langle\mathbb{A}_{\kappa}\left(\begin{array}{c}
\boldsymbol{\zeta}^{\perp} \\
\boldsymbol{\mu}^{0}
\end{array}\right),\left(\begin{array}{c}
\boldsymbol{\xi}^{0} \\
\boldsymbol{\lambda}^{\perp}
\end{array}\right)\right\rangle_{\boldsymbol{\tau} \times \boldsymbol{\tau}}+\left\langle\mathbb{A}_{\kappa}\left(\begin{array}{c}
\boldsymbol{\zeta}^{0} \\
\boldsymbol{\mu}^{\perp}
\end{array}\right),\left(\begin{array}{c}
\boldsymbol{\xi}^{\perp} \\
\boldsymbol{\lambda}^{0}
\end{array}\right)\right\rangle_{\boldsymbol{\tau} \times \boldsymbol{\tau}} .
\end{aligned}
$$

Let us take a look at the bilinear forms in the second line:

$$
\left\langle\mathbb{A}_{\kappa}\left(\begin{array}{c}
\boldsymbol{\zeta}^{\perp} \\
\boldsymbol{\mu}^{0}
\end{array}\right),\left(\begin{array}{c}
\boldsymbol{\xi}^{0} \\
\boldsymbol{\lambda}^{\perp}
\end{array}\right)\right\rangle_{\boldsymbol{\tau} \times \boldsymbol{\tau}}=\left\{\begin{array}{l}
\left\langle\mathbf{C}_{\kappa} \boldsymbol{\zeta}^{\perp}, \lambda^{\perp}\right\rangle_{\boldsymbol{\tau}, \Gamma}+\kappa\left\langle\mathbf{V}_{\kappa} \boldsymbol{\mu}^{0}, \boldsymbol{\lambda}^{\perp}\right\rangle_{\boldsymbol{\tau}, \Gamma}+ \\
+\kappa\left\langle\mathbf{V}_{\kappa} \boldsymbol{\zeta}^{\perp}, \boldsymbol{\xi}^{0}\right\rangle_{\boldsymbol{\tau}, \Gamma}+\left\langle\mathbf{C}_{\kappa} \boldsymbol{\mu}^{0}, \boldsymbol{\xi}^{0}\right\rangle_{\boldsymbol{\tau}, \Gamma}
\end{array}\right.
$$

Lemmas 9 and 12 show that this is a compact bilinear form! The same applies to the other term in the second line of (39). Harking back to the discussion in Sect. 3 , we emphasize that both Dirichlet and Neumann trace involve electric and magnetic components, which are isolated by the splitting:

$$
\text { Electric components: } \zeta^{0}, \mu^{\perp} \longleftrightarrow \text { Magnetic components: } \zeta^{\perp}, \mu^{0} .
$$

The bottom line is that up to compact terms electric and magnetic components of the traces are decoupled in $\mathbb{A}_{\kappa}$. It has turned out that the decoupling observed in the case of smooth boundaries does not reflect the "physics of the fields".

Using the appropriate splitting and decoupling, we can proceed as in the case of $\mathbf{S}_{0}$ : we introduce the isomorphism $\mathbb{X}_{\Gamma}: \boldsymbol{H}_{\times}^{-\frac{1}{2}}\left(\operatorname{div}_{\Gamma}, \Gamma\right)^{2} \mapsto \boldsymbol{H}_{\times}^{-\frac{1}{2}}\left(\operatorname{div}_{\Gamma}, \Gamma\right)^{2}$ by

$$
\mathbb{X}_{\Gamma}\left(\begin{array}{c}
\boldsymbol{\zeta} \\
\boldsymbol{\mu}
\end{array}\right):=\left(\begin{array}{c}
\mathbf{X}_{\Gamma} \boldsymbol{\zeta} \\
\mathbf{X}_{\Gamma} \boldsymbol{\mu}
\end{array}\right), \quad \boldsymbol{\zeta}, \boldsymbol{\mu} \in \boldsymbol{H}_{\times}^{-\frac{1}{2}}\left(\operatorname{div}_{\Gamma}, \Gamma\right) .
$$

Then we get the following generalization of Lemma 10.

Theorem 9 (Generalized Gårding inequality for $\mathbb{A}_{\kappa}$ ). There is a constant $C_{G}>0$ and a compact bilinear form $\mathbf{c}_{\Gamma}$ on $\boldsymbol{H}_{\times}^{-\frac{1}{2}}\left(\operatorname{div}_{\Gamma}, \Gamma\right) \times \boldsymbol{H}_{\times}^{-\frac{1}{2}}\left(\operatorname{div}_{\Gamma}, \Gamma\right)$ such that

$$
\left|\left\langle\mathbb{A}_{\kappa}\left(\begin{array}{c}
\boldsymbol{\zeta} \\
\boldsymbol{\mu}
\end{array}\right), \mathbb{X}_{\Gamma}\left(\begin{array}{c}
\overline{\boldsymbol{\zeta}} \\
\overline{\boldsymbol{\mu}}
\end{array}\right)\right\rangle_{\boldsymbol{\tau} \times \boldsymbol{\tau}}-\mathbf{c}_{\Gamma}\left(\left(\begin{array}{c}
\boldsymbol{\zeta} \\
\boldsymbol{\mu}
\end{array}\right),\left(\begin{array}{c}
\overline{\boldsymbol{\zeta}} \\
\overline{\boldsymbol{\mu}}
\end{array}\right)\right)\right| \geq C_{G}\left\|\left(\begin{array}{c}
\boldsymbol{\zeta} \\
\boldsymbol{\mu}
\end{array}\right)\right\|_{\boldsymbol{H}_{\times}^{-\frac{1}{2}}\left(\operatorname{div}_{\Gamma}, \Gamma\right)}^{2}
$$

for all $\boldsymbol{\zeta}, \boldsymbol{\mu} \in \boldsymbol{H}_{\times}^{-\frac{1}{2}}\left(\operatorname{div}_{\Gamma}, \Gamma\right)$. 
Proof. We have already found that up to compact perturbations

$$
\left\langle\mathbb{A}_{\kappa}\left(\begin{array}{c}
\boldsymbol{\zeta} \\
\boldsymbol{\mu}
\end{array}\right),\left(\begin{array}{c}
\boldsymbol{\xi} \\
\boldsymbol{\lambda}
\end{array}\right)\right\rangle_{\boldsymbol{\tau}, \Gamma} \simeq\left\langle\mathbb{A}_{\kappa}\left(\begin{array}{c}
\boldsymbol{\zeta}^{\perp} \\
\boldsymbol{\mu}^{0}
\end{array}\right),\left(\begin{array}{c}
\boldsymbol{\xi}^{\perp} \\
\boldsymbol{\lambda}^{0}
\end{array}\right)\right\rangle_{\boldsymbol{\tau}, \Gamma}+\left\langle\mathbb{A}_{\kappa}\left(\begin{array}{c}
\boldsymbol{\zeta}^{0} \\
\boldsymbol{\mu}^{\perp}
\end{array}\right),\left(\begin{array}{c}
\boldsymbol{\xi}^{0} \\
\boldsymbol{\lambda}^{\perp}
\end{array}\right)\right\rangle_{\boldsymbol{\tau}, \Gamma}
$$

What comes next amounts to reusing arguments from the proof of Lemma 10 . We inspect the first summand and find, using Lemmas 6 and 9,

$$
\begin{aligned}
\left\langle\mathbb{A}_{\kappa}\left(\begin{array}{c}
\boldsymbol{\zeta}^{\perp} \\
\boldsymbol{\mu}^{0}
\end{array}\right),\left(\begin{array}{c}
\overline{\boldsymbol{\zeta}}^{\perp} \\
-\overline{\boldsymbol{\mu}}^{0}
\end{array}\right)\right\rangle_{\boldsymbol{\tau} \times \boldsymbol{\tau}}= & -\left\langle\mathbf{C}_{\kappa} \boldsymbol{\zeta}^{\perp}, \overline{\boldsymbol{\mu}}^{0}\right\rangle_{\boldsymbol{\tau}, \Gamma}+\kappa\left\langle\mathbf{V}_{\kappa} \boldsymbol{\mu}^{0}, \overline{\boldsymbol{\mu}^{0}}\right\rangle_{\boldsymbol{\tau}, \Gamma}+ \\
& +\left\langle\mathbf{S}_{\kappa} \boldsymbol{\zeta}^{\perp}, \overline{\boldsymbol{\zeta}}^{\perp}\right\rangle_{\boldsymbol{\tau}, \Gamma}+\left\langle\mathbf{C}_{\kappa} \boldsymbol{\mu}^{0}, \overline{\boldsymbol{\zeta}}^{\perp}\right\rangle_{\boldsymbol{\tau}, \Gamma} \\
\simeq-2 i \operatorname{Im}\left\{\left\langle\mathbf{C}_{\kappa} \boldsymbol{\zeta}^{\perp}, \overline{\boldsymbol{\mu}}^{0}\right\rangle_{\boldsymbol{\tau}, \Gamma}\right\} & +\kappa\left\langle\mathbf{V}_{0} \boldsymbol{\mu}^{0}, \overline{\boldsymbol{\mu}^{0}}\right\rangle_{\boldsymbol{\tau}, \Gamma}+\frac{1}{\kappa}\left\langle V_{0}\left(\operatorname{div}_{\Gamma} \boldsymbol{\zeta}, \operatorname{div}_{\Gamma} \overline{\boldsymbol{\zeta}}\right\rangle_{\boldsymbol{\tau}, \Gamma} .\right.
\end{aligned}
$$

Appealing to Lemmas 2 and 8, we conclude that

$$
\mid\left\langle\mathbb{A}_{\kappa}\left(\begin{array}{c}
\boldsymbol{\zeta}^{\perp} \\
\boldsymbol{\mu}^{0}
\end{array}\right),\left(\begin{array}{c}
\overline{\boldsymbol{\zeta}}^{\perp} \\
-\overline{\boldsymbol{\mu}}^{0}
\end{array}\right)\right\rangle_{\boldsymbol{\tau} \times \boldsymbol{\tau}}+\text { comp. } \mid \geq C\left(\left\|\boldsymbol{\mu}^{0}\right\|_{\boldsymbol{H}_{\times}^{-\frac{1}{2}}(\Gamma)}^{2}+\left\|\boldsymbol{\zeta}^{\perp}\right\|_{\boldsymbol{H}_{\times}^{-\frac{1}{2}}\left(\operatorname{div}_{\Gamma}, \Gamma\right)}^{2}\right) .
$$

The same manipulations can be carried out for the second summand. Together with the stability of (21) this gives the assertion.

\section{Boundary Integral Equations}

Boundary integral equations (BIE) can be obtained in two ways, either by the $d i$ rect method or the indirect method. The distinct feature of the direct method is that traces of the solution of the transmission problem/boundary value problem occur as unknowns in the formulation. Its integral equations immediately arise from the Calderon projectors $\mathbb{P}_{\kappa}^{-}$and $\mathbb{P}_{\kappa}^{+}$via Theorem 8 . Conversely, the unknowns of the indirect methods are jumps of traces across $\Gamma$. It can be motivated by the fact that the potentials $\boldsymbol{\Psi}_{S L}^{\kappa}$ and $\boldsymbol{\Psi}_{D L}^{\kappa}$ already provide solutions to the homogeneous equations, $c f$. (29). An excellent presentation of the main ideas of indirect methods is given in [36, Vol. IV, Ch. XI].

\subsection{The direct method}

We start with the discussion of direct methods for scattering at a perfect conductor, that is, the exterior Dirichlet problem for the homogeneous electric wave equation

$$
\text { curl curl } \mathbf{e}-\kappa^{2} \mathbf{e}=0 \quad \text { in } \Omega^{\prime} \quad, \quad \gamma_{\mathbf{t}}^{+} \mathbf{e}=\gamma_{\mathbf{t}}^{+} \mathbf{e}_{i},
$$

plus Silver-Müller radiation conditions. We know that we can always find a unique solution of (41) [31, Thm. 6.10]. However, it is a bewildering feature of many boundary integral equations connected with (41) that they fail to have unique solutions, if $\kappa$ coincides with "forbidden wave numbers" [26,38]. Those are related to interior eigenvalues of the operator of (41). 
Definition 4. $\lambda \in \mathbb{R}$ is called an interior electric/magnetic Maxwell eigenvalue, if there is a non-zero $\mathbf{e} \in \boldsymbol{H}_{0}\left(\mathbf{c u r l} ; \Omega_{s}\right)$ or $\mathbf{e} \in \boldsymbol{H}\left(\mathbf{c u r l} ; \Omega_{s}\right)$, respectively, such that

$(\operatorname{curl} \mathbf{e}, \operatorname{curl} \mathbf{v})_{0 ; \Omega_{s}}=\lambda(\mathbf{e}, \mathbf{v})_{0 ; \Omega_{s}} \quad \forall \mathbf{v} \in \boldsymbol{H}_{0}\left(\operatorname{curl} ; \Omega_{s}\right)$ or $\mathbf{v} \in \boldsymbol{H}\left(\operatorname{curl} ; \Omega_{s}\right)$.

Note that these eigenvalues form a discrete sequence accumulating at $\infty$.

The first direct method relies on Theorem 8, which tells us that $\left(\gamma_{\mathbf{t}}^{+} \mathbf{e}_{i}, \boldsymbol{\lambda}\right)$ are exterior Cauchy data according to Definition 3, if $\mathbb{P}_{\kappa}^{-}\left(\gamma_{\mathbf{t}}^{+} \mathbf{e}_{i}, \boldsymbol{\lambda}\right)=0$. From the first row of this equation we obtain the integral equation of the first kind $\mathbf{S}_{\kappa} \boldsymbol{\lambda}=-\left(\frac{1}{2} \operatorname{Id}+\mathbf{C}_{\kappa}\right)\left(\gamma_{\mathbf{t}}^{+} \mathbf{e}_{i}\right)$ for the unknown Neumann data $\boldsymbol{\lambda}:=\gamma_{N}^{+} \mathbf{e}$ of a solution e of (41). In weak form it reads: seek $\boldsymbol{\lambda} \in \boldsymbol{H}_{\times}^{-\frac{1}{2}}\left(\operatorname{div}_{\Gamma}, \Gamma\right)$ such that

$\underset{\langle F| E}{\left\langle\mathbf{S}_{\kappa} \boldsymbol{\lambda}, \boldsymbol{\mu}\right\rangle_{\boldsymbol{\tau}, \Gamma}=-\left\langle\left(\frac{1}{2} \operatorname{Id}+\mathbf{C}_{\kappa}\right)\left(\gamma_{\mathbf{t}}^{+} \mathbf{e}_{i}\right), \boldsymbol{\mu}\right\rangle_{\boldsymbol{\tau}, \Gamma} \quad \forall \boldsymbol{\mu} \in \boldsymbol{H}_{\times}^{-\frac{1}{2}}\left(\operatorname{div}_{\Gamma}, \Gamma\right) .}$

Conversely, if $\left(\gamma_{\mathbf{t}}^{+} \mathbf{e}_{i}, \boldsymbol{\lambda}\right)$ satisfies (42), we find

$$
\mathbb{P}_{\kappa}^{-}\left(\begin{array}{c}
\gamma_{\mathbf{t}}^{+} \mathbf{e}_{i} \\
\boldsymbol{\lambda}
\end{array}\right)=\left(\begin{array}{l}
0 \\
\boldsymbol{\xi}
\end{array}\right) \quad \text { for some } \boldsymbol{\xi} \in \boldsymbol{H}_{\times}^{-\frac{1}{2}}\left(\operatorname{div}_{\Gamma}, \Gamma\right) .
$$

Hence, by Theorem $8, \xi$ is the Neumann trace of an electric eigenmode of $\Omega_{s}$. If $\kappa^{2}$ does not coincide with an interior electric eigenvalue, this eigenmode can only be trivial, which means $\boldsymbol{\xi}=0$. The next lemma summarizes our findings.

Lemma 13. Assume that $\kappa^{2}$ is not an interior electric eigenvalue. Then $\lambda \in$ $\boldsymbol{H}_{\times}^{-\frac{1}{2}}\left(\operatorname{div}_{\Gamma}, \Gamma\right)$ is a solution of (42) if and only if $\left(\gamma_{\mathbf{t}}^{+} \mathbf{e}_{i}, \boldsymbol{\lambda}\right)$ are Cauchy data for (41).

Remark 1. If $\kappa^{2}$ is an interior electric eigenvalue, then $\boldsymbol{\lambda}$ is unique up to Neumann traces $\boldsymbol{\xi}$ of the corresponding eigenmodes. Thanks to the representation formula (26), we find that $\boldsymbol{\Psi}_{S L}^{\kappa}(\boldsymbol{\xi})$ vanishes in $\Omega^{\prime}$. In other words, the representation

$$
\mathbf{e}=-\boldsymbol{\Psi}_{D L}^{\kappa}\left(\gamma_{\mathbf{t}}^{+} \mathbf{e}_{i}\right)-\boldsymbol{\Psi}_{S L}^{\kappa}(\boldsymbol{\lambda})
$$

will produce the unique field solution in $\Omega^{\prime}$.

Now, a standard Fredholm alternative argument can be applied:

Theorem 10. Provided that $\kappa$ satisfies the assumptions of Lemma 13, there exists a unique solution of (42) for any $\mathbf{e}_{i}$.

Using the second row of $\mathbb{P}_{\kappa}^{-}$we obtain the B.I.E. $\left(\frac{1}{2} \operatorname{Id}+\mathbf{C}_{\kappa}\right) \boldsymbol{\lambda}=-\mathbf{S}_{\kappa}\left(\gamma_{\mathbf{t}}^{+} \mathbf{e}_{i}\right)$, whose associated variational problem can be stated as: seek $\boldsymbol{\lambda} \in \boldsymbol{H}_{\times}^{-\frac{1}{2}}\left(\operatorname{div}_{\Gamma}, \Gamma\right)$ such that

$$
\left\langle\left(\frac{1}{2} \operatorname{Id}+\mathbf{C}_{\kappa}\right) \boldsymbol{\lambda}, \boldsymbol{\mu}\right\rangle_{\boldsymbol{\tau}, \Gamma}=-\left\langle\mathbf{S}_{\kappa}\left(\gamma_{\mathbf{t}}^{+} \mathbf{e}_{i}\right), \boldsymbol{\mu}\right\rangle_{\boldsymbol{\tau}, \Gamma} \quad \forall \boldsymbol{\mu} \in \boldsymbol{H}_{\times}^{-\frac{1}{2}}\left(\operatorname{div}_{\Gamma}, \Gamma\right) .
$$

In contrast to (42), in order to show unique solvability of (44) we need not only avoid "forbidden wave numbers", but have to assume smooth boundaries, too. 
Theorem 11. Let $\kappa^{2}$ be different from a interior magnetic eigenvalue. If $\Gamma$ is $C^{\infty}$ smooth, then (44) has a unique solution $\boldsymbol{\lambda} \in \boldsymbol{T} \boldsymbol{H}^{-\frac{1}{2}}\left(\operatorname{div}_{\Gamma} ; \Gamma\right)$ and the pair $\left(\gamma_{\mathbf{t}}^{+} \mathbf{e}^{i}, \boldsymbol{\lambda}\right)$ supplies Cauchy data for the electric wave equation in $\Omega^{\prime}$.

Proof. As in the justification of Lemma 13 it turns out that

$$
\mathbb{P}_{\kappa}^{-}\left(\begin{array}{c}
\gamma_{\mathbf{t}}^{+} \mathbf{e}_{i} \\
\boldsymbol{\lambda}
\end{array}\right)=\left(\begin{array}{l}
\boldsymbol{\xi} \\
0
\end{array}\right) \text { for some } \boldsymbol{\xi} \in \boldsymbol{T} \boldsymbol{H}^{-\frac{1}{2}}\left(\operatorname{div}_{\Gamma} ; \Gamma\right),
$$

if $\boldsymbol{\lambda}$ satisfies (44). By the assumption on $\kappa$ we have $\boldsymbol{\xi}=0$ and, by Theorem 8 , $\left(\gamma_{\mathbf{t}}^{+} \mathbf{e}_{i}, \boldsymbol{\lambda}\right)$ is identified as valid Maxwell Cauchy data for the exterior problem. Recalling the uniqueness result for (41), this means that solutions of (44) are unique. Next, use Lemma 11, which asserts the compactness of $\mathbf{C}_{\kappa}: \boldsymbol{T} \boldsymbol{H}^{-\frac{1}{2}}\left(\operatorname{div}_{\Gamma} ; \Gamma\right) \rightarrow$ $\boldsymbol{T} \boldsymbol{H}^{-\frac{1}{2}}\left(\operatorname{div}_{\Gamma} ; \Gamma\right)$. This confirms that the operator in (44) is Fredholm of index zero.

If $\Gamma \in C^{\infty}$ and $\gamma_{\mathbf{t}}^{+} \mathbf{e}_{i} \in \boldsymbol{H}_{\mathbf{t}}^{1}(\Gamma)$ (which, e.g., is fulfilled for exciting plane waves), the lifting properties of the operators $\mathbf{C}_{\kappa}$ according to Lemma 11 and the fact that $\operatorname{div}_{\Gamma} \circ \mathbf{S}_{\kappa}=\kappa \operatorname{div}_{\Gamma} \circ \mathbf{V}_{\kappa}$ bear out that the solution of (44) will belong to $\boldsymbol{T} \boldsymbol{H}\left(\operatorname{div}_{\Gamma}, \Gamma\right)$. Hence, a completely equivalent variational formulation in $\boldsymbol{T} \boldsymbol{H}\left(\operatorname{div}_{\Gamma}, \Gamma\right)$ is possible: find $\boldsymbol{\lambda} \in \boldsymbol{T} \boldsymbol{H}\left(\operatorname{div}_{\Gamma}, \Gamma\right)$ such that $\forall \boldsymbol{\mu} \in \boldsymbol{T} \boldsymbol{H}\left(\operatorname{div}_{\Gamma}, \Gamma\right)$

$$
\left(\left(\frac{1}{2} \operatorname{Id}+\mathbf{C}_{\kappa}\right) \boldsymbol{\lambda}, \boldsymbol{\mu}\right)_{\boldsymbol{T H}\left(\operatorname{div}_{\Gamma}, \Gamma\right)}=-\left(\mathbf{S}_{\kappa}\left(\gamma_{\mathbf{t}}^{+} \mathbf{e}_{i}\right), \boldsymbol{\mu}\right)_{\boldsymbol{T H}\left(\operatorname{div}_{\Gamma}, \Gamma\right)} .
$$

Given a sufficiently smooth $\gamma_{\mathbf{t}}^{+} \mathbf{e}_{i}$, the right hand side is a continuous functional on $\boldsymbol{T} \boldsymbol{H}\left(\operatorname{div}_{\Gamma}, \Gamma\right)$. In addition, Lemma 11 shows that $\mathbf{C}_{\kappa}: \boldsymbol{T H}\left(\operatorname{div}_{\Gamma}, \Gamma\right) \mapsto$ $\boldsymbol{T} \boldsymbol{H}^{1}\left(\operatorname{div}_{\Gamma}, \Gamma\right)$ and, hence, the sesqui-linear form in (45) turns out to be $\boldsymbol{T} \boldsymbol{H}\left(\operatorname{div}_{\Gamma}, \Gamma\right)$ coercive. Thus, Thm. 11 will remain valid for (45). The real rational behind the lifting of (44) into $\boldsymbol{T} \boldsymbol{H}\left(\operatorname{div}_{\Gamma}, \Gamma\right)$ will be elaborated in Sect. 9.

smallskip

Next, we tackle scattering at an isotropic, homogeneous dielectric object occupying $\Omega_{s}$. Inside $\Omega_{s}$ material parameters $\epsilon^{-}>0$ and $\mu^{-}>0$ prevail, leading to a wave number $\kappa^{-}:=\omega \sqrt{\epsilon^{-} \mu^{-}}$. Outside we face $\epsilon_{0}, \mu_{0}$ and wave number $\kappa^{+}$. These wave numbers underlie the definition of $\gamma_{N}^{-}$and $\gamma_{N}^{+}$. The transmission conditions from (1) become

$$
\gamma_{\mathbf{t}}^{-} \mathbf{e}=\gamma_{\mathbf{t}}^{+} \mathbf{e} \quad, \quad \frac{\kappa^{-}}{\mu^{-}} \gamma_{N}^{-} \mathbf{e}=\frac{\kappa^{+}}{\mu_{0}} \gamma_{N}^{+} \mathbf{e} .
$$

Taking our cue from the approach to acoustic scattering in [61], we introduce scaled boundary integral operators

$$
\widehat{\mathbb{A}}_{\kappa^{-}}=\left(\begin{array}{cc}
\operatorname{Id} & 0 \\
0 & \frac{\kappa^{-}}{\mu^{-}}
\end{array}\right) \mathbb{A}_{\kappa^{-}}\left(\begin{array}{cc}
\operatorname{Id} & 0 \\
0 & \frac{\mu^{-}}{\kappa^{-}}
\end{array}\right) \quad, \quad \widehat{\mathbb{A}}_{\kappa^{+}}=\left(\begin{array}{cc}
\operatorname{Id} & 0 \\
0 & \frac{\kappa^{+}}{\mu_{0}}
\end{array}\right) \mathbb{A}_{\kappa^{+}}\left(\begin{array}{cc}
\operatorname{Id} & 0 \\
0 & \frac{\mu_{0}}{\kappa^{+}}
\end{array}\right) .
$$

The following scaled traces match the scaled operators 


$$
\left(\boldsymbol{\zeta}^{+}, \boldsymbol{\lambda}^{+}\right)=\left(\gamma_{\mathbf{t}}^{+} \mathbf{e}, \frac{\kappa^{+}}{\mu_{0}} \gamma_{N}^{+} \mathbf{e}\right) \quad, \quad\left(\boldsymbol{\zeta}^{-}, \boldsymbol{\lambda}^{-}\right)=\left(\gamma_{\mathbf{t}}^{-} \mathbf{e}, \frac{\kappa^{-}}{\mu^{-}} \gamma_{N}^{-} \mathbf{e}\right)
$$

For them the transmission condition takes the simple form

$$
\left(\begin{array}{c}
\boldsymbol{\zeta}^{-} \\
\boldsymbol{\lambda}^{-}
\end{array}\right)-\left(\begin{array}{c}
\boldsymbol{\zeta}^{+} \\
\boldsymbol{\lambda}^{+}
\end{array}\right)=\left(\begin{array}{c}
\gamma_{\mathbf{t}}^{+} \mathbf{e}_{i} \\
\gamma_{\mathbf{t}}^{+} \mathbf{h}_{i}
\end{array}\right)
$$

A scaled version of Theorem 8 bears out that $\left(\boldsymbol{\zeta}^{-}, \boldsymbol{\lambda}^{-}\right)$and $\left(\boldsymbol{\zeta}^{+}, \boldsymbol{\lambda}^{+}\right)$are interior/exterior Cauchy data for the electric wave equation with wave numbers $\kappa^{-}$and $\kappa^{+}$, respectively, if and only if

$$
\left(\frac{1}{2} \operatorname{Id}-\widehat{\mathbb{A}}_{\kappa^{-}}\right)\left(\begin{array}{c}
\zeta^{-} \\
\lambda^{-}
\end{array}\right)=0 \quad, \quad\left(\frac{1}{2} \operatorname{Id}+\widehat{\mathbb{A}}_{\kappa^{+}}\right)\left(\begin{array}{c}
\boldsymbol{\zeta}^{+} \\
\lambda^{+}
\end{array}\right)=0
$$

Using (46), this immediately implies that

$$
\left(\widehat{\mathbb{A}}_{\kappa^{-}}+\widehat{\mathbb{A}}_{\kappa^{+}}\right)\left(\begin{array}{c}
\boldsymbol{\zeta}^{+} \\
\boldsymbol{\lambda}^{+}
\end{array}\right)=\left(\frac{1}{2} \mathrm{Id}-\widehat{\mathbb{A}}_{\kappa^{-}}\right)\left(\begin{array}{c}
\gamma_{\mathbf{t}}^{+} \mathbf{e}_{i} \\
\gamma_{\mathbf{t}}^{+} \mathbf{h}_{i}
\end{array}\right) .
$$

These are the boundary integral equations of the direct method for the transmission problem. Conversely, if $\left(\boldsymbol{\zeta}^{+}, \boldsymbol{\lambda}^{+}\right)$is a solution of (48), set $\left(\begin{array}{c}\boldsymbol{\zeta}^{-} \\ \boldsymbol{\lambda}^{-}\end{array}\right)=\left(\begin{array}{c}\boldsymbol{\zeta}^{+} \\ \boldsymbol{\lambda}^{+}\end{array}\right)+\left(\begin{array}{c}\gamma_{\mathbf{t}}^{+} \mathbf{e}_{i} \\ \gamma_{\mathbf{t}}^{+} \mathbf{h}_{i}\end{array}\right)$, and consider

$$
\left(\begin{array}{l}
\widetilde{\boldsymbol{\zeta}}^{-} \\
\tilde{\boldsymbol{\lambda}}^{-}
\end{array}\right):=\left(\frac{1}{2} \operatorname{Id}-\widehat{\mathbb{A}}_{\kappa^{-}}\right)\left(\begin{array}{l}
\boldsymbol{\zeta}^{-} \\
\boldsymbol{\lambda}^{-}
\end{array}\right) \quad, \quad\left(\begin{array}{l}
\widetilde{\boldsymbol{\zeta}}^{+} \\
\tilde{\boldsymbol{\lambda}}^{+}
\end{array}\right):=\left(\frac{1}{2} \operatorname{Id}+\widehat{\mathbb{A}}_{\kappa^{+}}\right)\left(\begin{array}{c}
\boldsymbol{\zeta}^{+} \\
\boldsymbol{\lambda}^{+}
\end{array}\right) .
$$

Owing to Thm. 8, the pairs $\left(\widetilde{\boldsymbol{\zeta}}^{-}, \widetilde{\boldsymbol{\lambda}}^{-}\right)$and $\left(\widetilde{\boldsymbol{\zeta}}^{+}, \widetilde{\boldsymbol{\lambda}}^{+}\right)$are Maxwell Cauchy data for $\Omega^{\prime}$ and $\Omega_{s}$ (and $\left.\kappa^{-}, \kappa^{+}\right)$, respectively. From equation (48) we infer that $\left(\widetilde{\zeta}^{-}, \widetilde{\boldsymbol{\lambda}}^{-}\right)=$ $\left(\widetilde{\zeta}^{+}, \widetilde{\lambda}^{+}\right)$. Thus, the interior and exterior Dirichlet and Neumann traces of the related Maxwell solutions agree. A combination of these Maxwell solutions solves the homogeneous electric wave equation (with $\kappa^{+}$inside $\Omega_{s}$ and $\kappa^{-}$outside) in $\mathbb{R}^{3}$ and satisfies the Silver-Müller radiation conditions. Thanks to the uniqueness of solutions of the exterior Maxwell problem, it has to vanish. This implies $\left(\widetilde{\boldsymbol{\zeta}}^{-}, \widetilde{\boldsymbol{\lambda}}^{-}\right)=0$ and $\left(\widetilde{\boldsymbol{\zeta}}^{+}, \widetilde{\boldsymbol{\lambda}}^{+}\right)=0$, so that we recover (48). This confirms the following result.

Lemma 14. Any solution $\left(\boldsymbol{\zeta}^{+}, \boldsymbol{\lambda}^{+}\right)$of (48) provides (scaled) exterior Cauchy data for the transmission problem with excitation by an incident wave $\left(\mathbf{e}_{i}, \mathbf{h}_{i}\right)$.

Using the pairing $\langle\cdot, \cdot\rangle_{\boldsymbol{\tau} \times \boldsymbol{\tau}}$, the variational formulation of (48) in $\boldsymbol{H}_{\times}^{-\frac{1}{2}}\left(\operatorname{div}_{\Gamma}, \Gamma\right) \times$ $\boldsymbol{H}_{\times}^{-\frac{1}{2}}\left(\operatorname{div}_{\Gamma}, \Gamma\right)$ is straightforward. So is the next theorem that arises from Thm. 9 , the previous Lemma, and a Fredholm argument.

Theorem 12. The boundary integral equation (48) has a unique solution for any excitation. 


\subsection{The indirect method}

We will only discuss the exterior Dirichlet problem for the electric wave equation. Let $\mathbf{e}^{+}$denote the unique solution of the exterior Dirichlet boundary value problem, satisfying $\gamma_{\mathbf{t}}^{+} \mathbf{e}^{+}=\gamma_{\mathbf{t}}^{+} \mathbf{e}_{i}$ and the Silver-Müller radiation conditions at $\infty$. Write $\mathbf{e}^{-}$for the solution of an interior Dirichlet problem for the electric wave equation, such that $\gamma_{\mathbf{t}}^{-} \mathbf{e}^{-}=\gamma_{\mathbf{t}}^{+} \mathbf{e}^{+}$. Again it is crucial to stay away from "forbidden wave numbers": let us assume that $\kappa^{2}$ does not coincide with an interior electric eigenvalue. Therefore, such an $\mathbf{e}^{-}$exists and is unique. Call $\mathbf{e}$ the Maxwell solution in $\Omega_{s} \cup \Omega^{\prime}$ that emerges by combining $\mathbf{e}^{+}$and $\mathbf{e}^{-}$. As $\left[\gamma_{\mathbf{t}}\right]_{\Gamma}(\mathbf{e})=0$, the representation formula (26) becomes

$$
\mathbf{e}=-\boldsymbol{\Psi}_{S L}^{\kappa}\left(\left[\gamma_{N}\right]_{\Gamma}(\mathbf{e})\right) \text { in } \boldsymbol{H}_{\mathrm{loc}}\left(\operatorname{curl}^{2}, \Omega_{s} \cup \Omega^{\prime}\right) .
$$

Applying the exterior Dirichlet trace $\gamma_{\mathbf{t}}^{+}$gives us the final integral equation in weak form: seek the unknown jump $\boldsymbol{\lambda}:=\left[\gamma_{N}\right]_{\Gamma}(\mathbf{e}) \in \boldsymbol{H}_{\times}^{-\frac{1}{2}}\left(\operatorname{div}_{\Gamma}, \Gamma\right)$, which satisfies

$$
\left\langle\mathbf{S}_{\kappa} \boldsymbol{\lambda}, \boldsymbol{\mu}\right\rangle_{\boldsymbol{\tau}, \Gamma}=-\left\langle\gamma_{\mathbf{t}}^{+} \mathbf{e}_{i}, \boldsymbol{\mu}\right\rangle_{\boldsymbol{\tau}, \Gamma} \quad \forall \boldsymbol{\mu} \in \boldsymbol{H}_{\times}^{-\frac{1}{2}}\left(\operatorname{div}_{\Gamma}, \Gamma\right) .
$$

This integral equation is also known as electric field integral equation (EFIE) or Rumsey's principle. Theorem 10 applies, because (42) and (49) feature the same bilinear form.

Parallel to the case of direct methods for the exterior Dirichlet problem, we have a second option also in the case of the indirect approach. We assume that $\kappa^{2}$ does not agree with an interior magnetic eigenvalue. Then, we may choose $\mathbf{e}^{-}$as a Neumann extension of $\mathbf{e}^{+}$, that is, $\mathbf{e}^{-}$is the solution of the interior Neumann problem for the electric wave equation with Neumann data $\gamma_{N}^{-} \mathbf{e}^{-}=\gamma_{N}^{+} \mathbf{e}^{+}$. Combining $\mathbf{e}^{+}$and $\mathbf{e}^{-}$to form $\mathbf{e}$, we conclude from (26) that $\mathbf{e}=-\boldsymbol{\Psi}_{D L}^{\kappa}\left(\left[\gamma_{\mathbf{t}}\right]_{\Gamma}(\mathbf{e})\right)$ in $\boldsymbol{H}_{\text {loc }}\left(\mathbf{c u r l}^{2}, \Omega_{s} \cup \Omega^{\prime}\right)$. Applying the exterior Dirichlet trace to this equation, we get the so-called magnetic field integral equation (MFIE), an integral equation of the second kind: find $\boldsymbol{\zeta} \in \boldsymbol{H}_{\times}^{-\frac{1}{2}}\left(\operatorname{div}_{\Gamma}, \Gamma\right)$ with

$$
\left\langle\left(\frac{1}{2} \operatorname{Id}-\mathbf{C}_{\kappa}\right) \boldsymbol{\zeta}, \boldsymbol{\mu}\right\rangle_{\boldsymbol{\tau}, \Gamma}=\left\langle\gamma_{\mathbf{t}}^{+} \mathbf{e}_{i}, \boldsymbol{\mu}\right\rangle_{\boldsymbol{\tau}, \Gamma} \quad \forall \boldsymbol{\mu} \in \boldsymbol{H}_{\times}^{-\frac{1}{2}}\left(\operatorname{div}_{\Gamma}, \Gamma\right) .
$$

Its theoretical analysis on smooth surfaces is already covered by Thm. 11 .

A serious drawback of the integral equations stated so far is their vulnerability to the presence of forbidden wave numbers, though the related boundary value problem always possesses a unique solution. Only one class of indirect BIE, the so-called combined field integral equations (CFIE), enjoys immunity. They owe their name to the fact that both $\boldsymbol{\Psi}_{D L}^{\kappa}$ and $\boldsymbol{\Psi}_{S L}^{\kappa}$ enter the trial expression for $\mathbf{e}$. A crucial prerequisite is a compact "smoothing operator" $\mathbf{M}: \boldsymbol{H}_{\times}^{-\frac{1}{2}}\left(\operatorname{div}_{\Gamma}, \Gamma\right) \mapsto \boldsymbol{H}_{\times}^{-\frac{1}{2}}\left(\operatorname{div}_{\Gamma}, \Gamma\right)$ that satisfies

$$
\boldsymbol{\mu} \in \boldsymbol{H}_{\times}^{-\frac{1}{2}}\left(\operatorname{div}_{\Gamma}, \Gamma\right): \quad\langle\mathrm{M} \boldsymbol{\mu}, \overline{\boldsymbol{\mu}}\rangle_{\boldsymbol{\tau}, \Gamma}>0 \quad \Leftrightarrow \quad \boldsymbol{\mu} \neq 0 .
$$


It is an important building block of the trial representation formula

$$
\mathbf{e}=-i \eta \boldsymbol{\Psi}_{S L}^{\kappa}(\boldsymbol{\zeta})-\boldsymbol{\Psi}_{D L}^{\kappa}(\mathrm{M} \boldsymbol{\zeta})
$$

where $\boldsymbol{\zeta} \in \boldsymbol{H}_{\times}^{-\frac{1}{2}}\left(\operatorname{div}_{\Gamma}, \Gamma\right), \eta>0$. By (29), this field is a Maxwell solution in $\Omega_{s} \cup \Omega^{\prime}$. The exterior Dirichlet trace applied to (51) results in the combined field integral equation: find $\boldsymbol{\zeta} \in \boldsymbol{H}_{\times}^{-\frac{1}{2}}\left(\operatorname{div}_{\Gamma}, \Gamma\right)$ such that $\forall \boldsymbol{\mu} \in \boldsymbol{H}_{\times}^{-\frac{1}{2}}\left(\operatorname{div}_{\Gamma}, \Gamma\right)$

$$
-i\left\langle\eta \mathbf{S}_{\kappa}(\boldsymbol{\zeta}), \boldsymbol{\mu}\right\rangle_{\boldsymbol{\tau}, \Gamma}+\left\langle\left(\frac{1}{2} \operatorname{Id}-\mathbf{C}_{\kappa}\right)(\mathrm{M} \boldsymbol{\zeta}), \boldsymbol{\mu}\right\rangle_{\boldsymbol{\tau}, \Gamma}=\left\langle\gamma_{\mathbf{t}}^{+} \mathbf{e}_{i}, \boldsymbol{\mu}\right\rangle_{\boldsymbol{\tau}, \Gamma} .
$$

The idea to use a regularizing operator to state a combined field integral equation is due to Kress [48].

Theorem 13. The boundary integral equation (52) has a unique solution $\zeta \in$ $\boldsymbol{H}_{\times}^{-\frac{1}{2}}\left(\operatorname{div}_{\Gamma}, \Gamma\right)$ for all $\eta>0, \kappa>0$.

Proof. To demonstrate uniqueness, we assume that $\boldsymbol{\zeta} \in \boldsymbol{H}_{\times}^{-\frac{1}{2}}\left(\operatorname{div}_{\Gamma}, \Gamma\right)$ solves

$$
-i \eta \mathbf{S}_{\kappa}(\boldsymbol{\zeta})+\left(\frac{1}{2} \mathrm{Id}-\mathbf{C}_{\kappa}\right)(\mathrm{M} \boldsymbol{\zeta})=0 .
$$

It is immediate from the jump relations that e given by (51) is an exterior Maxwell solution with $\gamma_{\mathbf{t}}^{+} \mathbf{e}=0$. By uniqueness we infer that $\mathbf{e}=0$ in $\Omega^{\prime}$. Appealing to the jump relations from Theorem 7 once more, we find

$$
\gamma_{\mathbf{t}}^{-} \mathbf{e}=-\mathbf{M \zeta} \quad, \quad \gamma_{N}^{-} \mathbf{e}=-i \eta \zeta .
$$

Next, we use (11) and see that

$$
i \eta\langle\boldsymbol{\zeta}, \overline{\mathrm{M} \zeta}\rangle_{\boldsymbol{\tau}, \Gamma}=\left\langle\gamma_{N}^{-} \mathbf{e}, \overline{\gamma_{\mathbf{t}}^{-} \mathbf{e}}\right\rangle_{\boldsymbol{\tau}, \Gamma}=\int_{\Omega_{s}} \frac{1}{\kappa}|\mathbf{c u r l} \mathbf{e}|^{2} \mathrm{~d} \mathbf{x}-\kappa|\mathbf{e}|^{2} \mathrm{~d} \mathbf{x} \in \mathbb{R}
$$

Necessarily, $(\boldsymbol{\zeta}, \overline{\mathrm{M} \zeta})_{\tau}=0$, so that the requirements on $\mathrm{M}$ imply $\boldsymbol{\zeta}=0$.

Knowing that $\mathrm{M}$ is compact, we conclude from Lemma 10 that the bilinear form of (52) satisfies a generalized Gårding inequality. Thus, Thm. 4 gives existence from uniqueness.

A possible candidate for $\mathrm{M}$ can be introduced through a variational definition: for $\boldsymbol{\zeta} \in \boldsymbol{H}_{\times}^{-\frac{1}{2}}\left(\operatorname{div}_{\Gamma}, \Gamma\right)$ and all $\mathbf{q} \in \boldsymbol{H}_{\times}\left(\operatorname{div}_{\Gamma}, \Gamma\right), \mathbf{M} \boldsymbol{\zeta} \in \boldsymbol{H}_{\times}\left(\operatorname{div}_{\Gamma}, \Gamma\right)$ is to satisfy

$$
\langle\mathrm{M} \boldsymbol{\zeta}, \mathbf{q}\rangle_{0 ; \Gamma}+\left\langle\operatorname{div}_{\Gamma} \mathrm{M} \boldsymbol{\zeta}, \operatorname{div}_{\Gamma} \mathbf{q}\right\rangle_{0 ; \Gamma}=\langle\mathbf{q}, \boldsymbol{\zeta}\rangle_{\boldsymbol{\tau}, \Gamma}
$$

Obviously, $\mathrm{M}: \boldsymbol{H}_{\times}^{-\frac{1}{2}}\left(\operatorname{div}_{\Gamma}, \Gamma\right) \mapsto \boldsymbol{H}_{\times}\left(\operatorname{div}_{\Gamma}, \Gamma\right)$ is a continuous linear operator. By density of $\boldsymbol{H}_{\times}\left(\operatorname{div}_{\Gamma}, \Gamma\right)$ in $\boldsymbol{H}_{\times}^{-\frac{1}{2}}\left(\operatorname{div}_{\Gamma}, \Gamma\right), \mathrm{M}$ must be injective, which also means

$$
\langle\mathrm{M} \zeta, \overline{\boldsymbol{\zeta}}\rangle_{\boldsymbol{\tau}, \Gamma}=\|\mathrm{M} \boldsymbol{\zeta}\|_{\boldsymbol{H}_{\times}\left(\operatorname{div}_{\Gamma}, \Gamma\right)}^{2}>0 \Leftrightarrow \quad \boldsymbol{\zeta} \neq 0 .
$$

It is easy to see that $\mathrm{M}$ inherits compactness from the embedding $\boldsymbol{H}_{\times}\left(\operatorname{div}_{\Gamma}, \Gamma\right) \hookrightarrow$ $\boldsymbol{H}_{\times}^{-\frac{1}{2}}\left(\operatorname{div}_{\Gamma}, \Gamma\right)$. 


\section{Boundary Element Spaces}

We equip the piecewise smooth compact two-dimensional surface $\Gamma$ with an oriented triangulation $\Gamma_{h}$. This means that all its edges are endowed with a direction. We assume a perfect resolution of $\Gamma$, that is $\Gamma=\bar{K}_{1} \cup \ldots \cup \bar{K}_{N}$, where $\mathcal{K}_{h}:=\left\{K_{1}, \ldots, K_{N}\right\}$ is the set of mutually disjoint open cells of $\Gamma_{h}$. Moreover, no cell may straddle boundaries of the smooth faces $\Gamma^{j}$ of $\Gamma$. We will admit triangular and quadrilateral cells only: for each $K \in \mathcal{K}_{h}$ there is a diffeomorphism $\boldsymbol{\Phi}_{K}: \widehat{K} \mapsto \bar{K}$, where $\widehat{K}$ is the "unit triangle" or unit square in $\mathbb{R}^{2}$, depending on the shape of $K$ [27, Sect. 5].

This paves the way for a parametric construction of boundary elements: to begin with, choose finite-dimensional local spaces $\mathcal{W}(\widehat{K}) \subset\left(C^{\infty}(\widehat{K})\right)^{2}$ of polynomial vectorfields together with a dual basis of so-called local degrees of freedom (d.o.f.). Possible choices for $\mathcal{W}(\widehat{K})$ and related d.o.f. abound: we may use the classical triangular Raviart-Thomas $\left(\mathrm{RT}_{p}\right)$ elements of polynomial order $p \in \mathbb{N}_{0}$ [56],

$$
\mathcal{W}(\widehat{K}):=\left\{\mathbf{x} \mapsto \mathbf{p}_{1}(\mathbf{x})+p_{2}(\mathbf{x}) \cdot \mathbf{x}, \mathbf{x} \in \widehat{K}, \mathbf{p}_{1} \in\left(\mathcal{P}_{p}(\widehat{K})\right)^{2}, p_{2} \in \mathcal{P}_{p}(\widehat{K})\right\}
$$

where $\mathcal{P}_{p}(\widehat{K})$ is the space of two-variable polynomials of total degree $\leq p$. An alternative are the triangular $\mathrm{BDM}_{p}$ elements of degree $p$ [12], $p \in \mathbb{N}_{0}$, which rely on $\mathcal{W}(\widehat{K}):=\left(\mathcal{P}_{p+1}(\widehat{K})\right)^{2}$. In both cases, the usual d.o.f. involve certain polynomial moments of normal components on edges, together with interior vectorial moments for $p>0$. For instance, in the case of $\mathrm{RT}_{0}$, edge fluxes are the appropriate degrees of freedom:

$$
\boldsymbol{\mu}_{h} \in \mathcal{W}(\widehat{K}) \mapsto \int_{\widehat{e}} \boldsymbol{\mu}_{h} \cdot \widehat{\mathbf{n}} \mathrm{d} S, \quad \widehat{e} \text { edge of } \widehat{K}
$$

Similar local spaces and degrees of freedom are available for the unit square.

Using the pull-back of 1-forms the local spaces can be lifted to the cells of $\Gamma_{h}$. In terms of vectorfields this is equivalent to the Piola transformation

$$
\left(\mathfrak{F}_{K} \boldsymbol{\mu}\right)(\mathbf{x}):=\sqrt{\operatorname{det}(\mathrm{G})} \mathrm{G}^{-1} D \boldsymbol{\Phi}_{K}^{T}(\widehat{\mathbf{x}}) \boldsymbol{\mu}(\widehat{\mathbf{x}}),
$$

where $\mathrm{G}:=D \boldsymbol{\Phi}(\widehat{\mathbf{x}})^{T} D \boldsymbol{\Phi}(\widehat{\mathbf{x}}), \mathbf{x}=\boldsymbol{\Phi}_{K}(\widehat{\mathbf{x}}), \widehat{\mathbf{x}} \in \widehat{K}$. Thus, we can introduce the global boundary element space

$$
\mathcal{W}_{h}:=\left\{\boldsymbol{\mu} \in \boldsymbol{H}_{\times}\left(\operatorname{div}_{\Gamma}, \Gamma\right): \boldsymbol{\mu}_{\mid K} \in \mathfrak{F}_{K}(\mathcal{W}(\widehat{K})) \forall K \in \mathcal{K}_{h}\right\}
$$

In practice, $\mathcal{W}_{h} \subset \boldsymbol{H}_{\times}\left(\operatorname{div}_{\Gamma}, \Gamma\right)$ is ensured by a suitable choice of d.o.f. Remember that d.o.f. have to be associated with individual edges of $\widehat{K}$ or the interior of $\widehat{K}$. It is crucial that the normal component of any $\widehat{\boldsymbol{\mu}}_{h} \in \mathcal{W}(\widehat{K})$ on any edge $\widehat{e}$ of $\widehat{K}$ vanishes if and only if $\widehat{\boldsymbol{\mu}}_{h}$ belongs to the kernel of all local d.o.f. associated with $\widehat{e}$. In light of (12), this ensures $\mathcal{W} \subset \boldsymbol{H}_{\times}\left(\operatorname{div}_{\Gamma}, \Gamma\right)$. In the sequel $\boldsymbol{W}_{h}$ will designate a generic $\boldsymbol{H}_{\times}\left(\operatorname{div}_{\Gamma}, \Gamma\right)$-conforming boundary element space. It may arise from the $\mathrm{RT}_{p}$ family of elements, $p \in \mathbb{N}_{0}$, the $\mathrm{BDM}_{p}$ family, or a combination of both. 
Based on the degrees of freedom we can introduce local interpolation operators $\Pi_{h}: \operatorname{Dom}\left(\Pi_{h}\right) \mapsto \mathcal{W}_{h}$. It is a projector onto $\mathcal{W}_{h}$ and enjoys the fundamental commuting diagram property

$$
\operatorname{div}_{\Gamma} \circ \Pi_{h}=\mathrm{Q}_{h} \circ \operatorname{div}_{\Gamma} \quad \text { on } \boldsymbol{H}_{\times}\left(\operatorname{div}_{\Gamma}, \Gamma\right) \cap \operatorname{Dom}\left(\Pi_{h}\right) .
$$

Here, $\mathrm{Q}_{h}$ is the $L^{2}(\Gamma)$-orthogonal projection onto a suitable space $Q_{h}$ of $\Gamma_{h^{-}}$ piecewise polynomial discontinuous functions. It must be emphasized that the interpolation operators $\Pi_{h}$ fail to be bounded on $\boldsymbol{H}_{\times}\left(\operatorname{div}_{\Gamma}, \Gamma\right)$; slightly more regularity of tangential vectorfields in $\operatorname{Dom}\left(\Pi_{h}\right)$ is required [7, Lemma 4.7].

Next, we turn our attention to asymptotic properties of the boundary element spaces, in particular to estimates of interpolation errors and best approximation errors. We restrict ourselves to the $h$-version of boundary elements, which relies on shape-regular families $\left\{\Gamma_{h}\right\}_{h \in \mathbb{H}}$ of triangulations of $\Gamma[30$, Ch. $3, \S 3.1]$. Here, $\mathbb{H}$ stands for a decreasing sequence of meshwidths, and $\mathbb{H}$ is assumed to converge to zero.

By means of transformation to reference elements, the commuting diagram property, and Bramble-Hilbert arguments, interpolation error estimates can easily be obtained [13, III.3.3].

Lemma 15 (Interpolation error estimate). For $0<s \leq p+1$ we find constants $C>0$, depending only on the shape regularity of the meshes and such that for all $\boldsymbol{\mu} \in \boldsymbol{H}_{\times}^{s}(\Gamma) \cap \boldsymbol{H}_{\times}\left(\operatorname{div}_{\Gamma}, \Gamma\right), h \in \mathbb{H}$,

$$
\left\|\boldsymbol{\mu}-\Pi_{h} \boldsymbol{\mu}\right\|_{\boldsymbol{L}^{2}(\Gamma)} \leq C h^{s}\left(\|\boldsymbol{\mu}\|_{\boldsymbol{H}_{\times}^{s}(\Gamma)}+\left\|\operatorname{div}_{\Gamma} \boldsymbol{\mu}\right\|_{L^{2}(\Gamma)}\right),
$$

and such that for all $\boldsymbol{\mu} \in \boldsymbol{H}_{\times}\left(\operatorname{div}_{\Gamma}, \Gamma\right), \operatorname{div}_{\Gamma} \boldsymbol{\mu} \in H_{-}^{s}(\Gamma)$

$$
\left\|\operatorname{div}_{\Gamma}\left(\boldsymbol{\mu}-\Pi_{h} \boldsymbol{\mu}\right)\right\|_{L^{2}(\Gamma)} \leq C h^{s}\left\|\operatorname{div}_{\Gamma} \boldsymbol{\mu}\right\|_{H_{-}^{s}(\Gamma)} .
$$

Corollary 5. The union of all boundary element spaces $\mathcal{W}_{h}, h \in \mathbb{H}$, is dense in $\boldsymbol{H}_{\times}^{-\frac{1}{2}}\left(\operatorname{div}_{\Gamma}, \Gamma\right)$.

A particular variant of the above interpolation error estimate addresses vector fields with discrete surface divergence:

Lemma 16. If $\boldsymbol{\mu} \in \boldsymbol{H}_{\times}^{s}(\Gamma), 0<s \leq 1$, and $\operatorname{div}_{\Gamma} \boldsymbol{\mu} \in Q_{h}$, then there is a constant $C>0$, depending on the shape-regularity of the meshes only, such that

$$
\left\|\boldsymbol{\mu}-\Pi_{h} \boldsymbol{\mu}\right\|_{\boldsymbol{L}_{\mathbf{t}}^{2}(\Gamma)} \leq C h^{s}\|\boldsymbol{\mu}\|_{\boldsymbol{H}_{\times}^{s}(\Gamma)} .
$$

¿From the interpolation error estimates we instantly get best approximation estimates in terms of the $\boldsymbol{H}_{\times}\left(\operatorname{div}_{\Gamma}, \Gamma\right)$-norm. Yet, what we actually need is a result about approximation in the "energy norm" $\|\cdot\|_{\boldsymbol{H}_{\times}^{-\frac{1}{2}}\left(\operatorname{div}_{\Gamma}, \Gamma\right)}$ of the form

$$
\inf _{\boldsymbol{\xi}_{h}}\left\|\boldsymbol{\mu}_{h}-\boldsymbol{\xi}_{h}\right\|_{\boldsymbol{H}_{\times}^{-\frac{1}{2}}\left(\operatorname{div}_{\Gamma}, \Gamma\right)} \leq C h^{s+\frac{1}{2}}\|\boldsymbol{\mu}\|_{\boldsymbol{H}_{\times}^{s}\left(\operatorname{div}_{\Gamma}, \Gamma\right)} .
$$


The estimate in $\boldsymbol{H}_{\times}\left(\operatorname{div}_{\Gamma}, \Gamma\right)$ does not directly provide (58). Even worse, standard duality arguments cannot be applied. Recall their main idea: we set out from a Hilbertian triple $V \subset H \subset V^{\prime}$, have a finite dimensional subspace of $H$, say $V_{h}$, and we want to estimate the best approximation error in $V^{\prime}$. Then it is crucial that we know how to use the difference in regularity between $H$ and $V$ through an estimate of the type $\exists v_{h} \in V_{h}:\left\|u-v_{h}\right\|_{H} \leq C(h)\|u\|_{V}$, with $C(h)$ optimal in a suitable sense.

Here, we have an estimate between $\boldsymbol{H}_{\times}\left(\operatorname{div}_{\Gamma}, \Gamma\right)$ and $\boldsymbol{H}_{\times}^{s}\left(\operatorname{div}_{\Gamma}, \Gamma\right), s>0$. Thus, we should use $\boldsymbol{H}_{\times}\left(\operatorname{div}_{\Gamma}, \Gamma\right)$ as self dual space, i.e., the standard inner product in $\boldsymbol{H}_{\times}\left(\operatorname{div}_{\Gamma}, \Gamma\right)$. But, in order to conclude, we should be able to prove that $\boldsymbol{H}_{\times}^{-s}\left(\operatorname{div}_{\Gamma}, \Gamma\right)$ is dual of $\boldsymbol{H}_{\times}^{s}\left(\operatorname{div}_{\Gamma}, \Gamma\right)$ for $0<s \leq \frac{1}{2}$ with respect to the $\boldsymbol{H}_{\times}\left(\operatorname{div}_{\Gamma}, \Gamma\right)$ inner product. Unfortunately this is the case for regular surfaces but not for non-regular ones [29].

The question of obtaining (58) has been addressed in [16] and the idea is to use the duality argument face by face (which are seen as regular open manifolds), exploiting continuity of the normal components of vector-fields in $\boldsymbol{H}_{\times}\left(\operatorname{div}_{\Gamma}, \Gamma\right)$. At the end of a technical procedure we obtain the following result:

Theorem 14. Let $\mathcal{P}_{h}: \boldsymbol{H}_{\times}^{-\frac{1}{2}}\left(\operatorname{div}_{\Gamma}, \Gamma\right) \rightarrow \mathcal{W}_{h}$ be the orthogonal projection with respect to the $\boldsymbol{H}_{\times}^{-\frac{1}{2}}\left(\operatorname{div}_{\Gamma}, \Gamma\right)$ inner product. Then, for any $-\frac{1}{2} \leq s \leq p+1$ we have

$$
\left\|\boldsymbol{\mu}-\mathcal{P}_{h} \boldsymbol{\mu}\right\|_{\boldsymbol{H}_{\times}^{-\frac{1}{2}}\left(\operatorname{div}_{\Gamma}, \Gamma\right)} \leq C h^{s+\frac{1}{2}}\|\boldsymbol{\mu}\|_{\boldsymbol{H}_{\times}^{s}\left(\operatorname{div}_{\Gamma}, \Gamma\right)} \quad \forall \boldsymbol{\mu} \in \boldsymbol{H}_{\times}^{s}\left(\operatorname{div}_{\Gamma}, \Gamma\right) .
$$

This theorem tells us that we can expect good approximation properties, but these cannot be obtained using local interpolation operators.

\section{Galerkin Discretization}

The Galerkin approach simply consists of replacing the Hilbert spaces $\boldsymbol{H}_{\times}^{-\frac{1}{2}}\left(\operatorname{div}_{\Gamma}, \Gamma\right)$ and $\boldsymbol{H}_{\times}\left(\operatorname{div}_{\Gamma}, \Gamma\right)$ in the variational formulations by finite dimensional subspaces $\mathcal{W}_{h}$.

\subsection{Integral equations of the first kind}

First, we study the simplest BIE of the first kind, namely the electric field integral equations (42) and (49), that is, we examine variational problems like: seek $\boldsymbol{\lambda} \in$ $\boldsymbol{H}_{\times}^{-\frac{1}{2}}\left(\operatorname{div}_{\Gamma}, \Gamma\right)$ such that

$$
\text { EFIE: } a(\boldsymbol{\lambda}, \boldsymbol{\mu}):=\left\langle\mathbf{S}_{\kappa} \boldsymbol{\lambda}, \boldsymbol{\mu}\right\rangle_{\boldsymbol{\tau}, \Gamma}=\operatorname{r.h.s.(\boldsymbol {\mu })} \forall \boldsymbol{\mu} \in \boldsymbol{H}_{\times}^{-\frac{1}{2}}\left(\operatorname{div}_{\Gamma}, \Gamma\right),
$$

for a suitable continuous functional on the right hand side. If $\kappa$ stays away from interior electric Maxwell eigenvalues, we saw that the operator $\mathbf{S}_{\kappa}: \boldsymbol{H}_{\times}^{-\frac{1}{2}}\left(\operatorname{div}_{\Gamma}, \Gamma\right) \mapsto$ 
$\boldsymbol{H}_{\times}^{-\frac{1}{2}}\left(\operatorname{div}_{\Gamma}, \Gamma\right)$ defines an isomorphism. This is equivalent to the existence of a constant $C_{S}>0$ such that the following continuous inf-sup condition holds true:

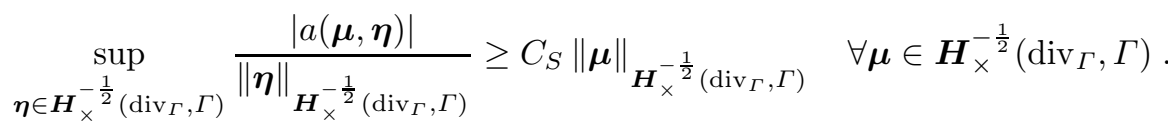

We aim at establishing a uniform discrete inf-sup-condition of the form: there exists $C_{D}>0$ such that $\forall \boldsymbol{\mu}_{h} \in \mathcal{W}_{h}$,

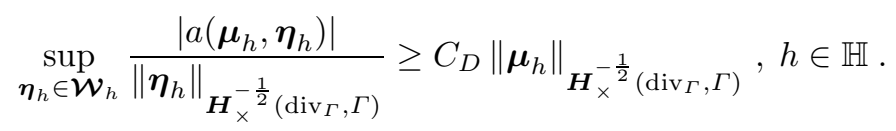

According to Babuška's theory [8] refined in [63] this guarantees existence of discrete solutions $\boldsymbol{\lambda}_{h} \in \mathcal{W}_{h}$ and translates into their quasi-optimal behaviour:

$$
\left\|\boldsymbol{\lambda}-\boldsymbol{\lambda}_{h}\right\|_{\boldsymbol{H}_{\times}^{-\frac{1}{2}}\left(\operatorname{div}_{\Gamma}, \Gamma\right)} \leq C_{D}^{-1} C_{A} \inf _{\boldsymbol{\eta}_{h} \in \mathcal{W}}\left\|\boldsymbol{\lambda}-\boldsymbol{\eta}_{h}\right\|_{\boldsymbol{H}_{\times}^{-\frac{1}{2}}\left(\operatorname{div}_{\Gamma}, \Gamma\right)} \quad \forall h \in \mathbb{H},
$$

where $C_{A}>0$ is the operator norm of $a(\cdot, \cdot)$. As a first step towards a discrete inf-sup condition, we have to find a suitable candidate for $\boldsymbol{\eta}$ in (61). To that end, introduce the operator $\mathrm{T}: \boldsymbol{H}_{\times}^{-\frac{1}{2}}\left(\operatorname{div}_{\Gamma}, \Gamma\right) \mapsto \boldsymbol{H}_{\times}^{-\frac{1}{2}}\left(\operatorname{div}_{\Gamma}, \Gamma\right)$ through

$$
a(\boldsymbol{\eta}, \top \boldsymbol{\mu})=c_{\Gamma}(\boldsymbol{\mu}, \boldsymbol{\eta}) \quad \forall \boldsymbol{\eta} \in \boldsymbol{H}_{\times}^{-\frac{1}{2}}\left(\operatorname{div}_{\Gamma}, \Gamma\right), \boldsymbol{\mu} \in \boldsymbol{H}_{\times}^{-\frac{1}{2}}\left(\operatorname{div}_{\Gamma}, \Gamma\right),
$$

where $c_{\Gamma}$ is the compact bilinear form of Lemma 10. Owing to (61) this is a valid definition of a compact operator T. It is immediate from (61) and Lemma 10 that

$$
\left|a\left(\boldsymbol{\mu},\left(\mathbf{X}_{\Gamma}+\mathrm{T}\right) \overline{\boldsymbol{\mu}}\right)\right|=\left|a\left(\boldsymbol{\mu}, \mathbf{X}_{\Gamma} \overline{\boldsymbol{\mu}}\right)+c_{\Gamma}(\boldsymbol{\mu}, \overline{\boldsymbol{\mu}})\right| \geq C_{G}\|\boldsymbol{\mu}\|_{\boldsymbol{H}_{\times}^{-\frac{1}{2}}\left(\operatorname{div}_{\Gamma}, \Gamma\right)}^{2}
$$

for all $\boldsymbol{\mu} \in \boldsymbol{H}_{\times}^{-\frac{1}{2}}\left(\operatorname{div}_{\Gamma}, \Gamma\right)$. The choice $\boldsymbol{\eta}:=\left(\mathrm{X}_{\Gamma}+\mathrm{T}\right) \boldsymbol{\mu}$ will make (61) hold with $C_{S}=C_{G}$. The challenge is that $\left(\mathrm{X}_{\Gamma}+\mathrm{T}\right) \boldsymbol{\mu}_{h}$ will not be a boundary element function even for $\boldsymbol{\mu}_{h} \in \mathcal{W}_{h}$. This is clear because neither $\mathrm{X}_{\Gamma}$ nor $\mathrm{T}$ may leave the boundary element spaces invariant. It will be necessary to project $\mathrm{X}_{\Gamma} \boldsymbol{\mu}_{h}$ and $\mathrm{T} \boldsymbol{\mu}_{h}$ back to $\mathcal{W}_{h}$. This can be achieved by applying suitable continuous projection operators $\mathrm{P}_{h}^{X}: \mathrm{X}_{\Gamma}\left(\mathcal{W}_{h}\right) \mapsto \mathcal{W}_{h}, \mathrm{P}_{h}^{T}: \boldsymbol{H}_{\times}^{-\frac{1}{2}}\left(\operatorname{div}_{\Gamma}, \Gamma\right) \mapsto \mathcal{W}_{h}$. Then, for an arbitrary $\boldsymbol{\mu}_{h} \in \mathcal{W}_{h}$ we can hope that $\boldsymbol{\eta}_{h}:=\left(\mathrm{P}_{h}^{X} \circ \mathrm{X}_{\Gamma}+\mathrm{P}_{h}^{T} \circ \mathrm{T}\right) \boldsymbol{\mu}_{h}$ is an appropriate choice for $\boldsymbol{\eta}_{h}$ in (62). Making use of (64) we see that

$$
\left|a\left(\boldsymbol{\mu}_{h}, \boldsymbol{\eta}_{h}\right)\right|=\left|a\left(\boldsymbol{\mu}_{h},\left(\mathbf{X}_{\Gamma}+\mathrm{T}\right) \boldsymbol{\mu}_{h}\right)-a\left(\boldsymbol{\mu}_{h},\left(\left(\mathrm{Id}-\mathrm{P}_{h}^{X}\right) \mathbf{X}_{\Gamma}+\left(\mathrm{Id}-\mathrm{P}_{h}^{T}\right) \mathrm{T}\right) \boldsymbol{\mu}_{h}\right)\right|
$$

We know that $\left|a\left(\boldsymbol{\mu}_{h},\left(\mathrm{X}_{\Gamma}+\mathrm{T}\right) \boldsymbol{\mu}_{h}\right)\right| \geq C_{G}\left\|\boldsymbol{\mu}_{h}\right\|_{\boldsymbol{H}(\operatorname{curl} ; \Omega)}^{2}$ and we need to estimate the second term in the left hand side by the triangle inequality. Obviously, the projectors $\mathrm{P}_{h}^{X}, \mathrm{P}_{h}^{T}$ have to guarantee uniform convergence $\left(\mathrm{Id}-\mathrm{P}_{h}^{T}\right) \circ \mathrm{T}_{\mid \mathcal{W}_{h}} \rightarrow 0$ and 
$\left(\mathrm{Id}-\mathrm{P}_{h}^{X}\right) \circ \mathrm{X}_{\Gamma} \boldsymbol{\mathcal { W }}_{h} \rightarrow 0$ in $\boldsymbol{H}_{\times}^{-\frac{1}{2}}\left(\operatorname{div}_{\Gamma}, \Gamma\right)$ as $h \rightarrow 0$. For $\mathrm{P}_{h}^{T}$ this is easy: we choose $\mathrm{P}_{h}^{T}$ as the $\boldsymbol{H}_{\times}^{-\frac{1}{2}}\left(\operatorname{div}_{\Gamma}, \Gamma\right)$-orthogonal projection. Due to the compactness of $\mathrm{T}$, we know [49, Corollary 10.4] that there exists a decreasing function $\epsilon=\epsilon(h)$ such that $\lim _{h \rightarrow 0} \epsilon(h)=0$ and, for all $\boldsymbol{\mu}_{h} \in \boldsymbol{H}_{\times}^{-\frac{1}{2}}\left(\operatorname{div}_{\Gamma}, \Gamma\right)$,

$$
\left\|\left(\operatorname{Id}-\mathrm{P}_{h}^{T}\right) \circ \mathrm{T} \boldsymbol{\mu}_{h}\right\|_{\boldsymbol{H}_{\times}^{-\frac{1}{2}}\left(\operatorname{div}_{\Gamma}, \Gamma\right)}<\epsilon(h)\left\|\boldsymbol{\mu}_{h}\right\|_{\boldsymbol{H}_{\times}^{-\frac{1}{2}}\left(\operatorname{div}_{\Gamma}, \Gamma\right)} .
$$

As regards $\mathrm{P}_{h}^{X}$, a crucial hint lies in the observation that $\mathrm{P}_{h}^{X}$ acts on functions in $\mathrm{X}_{\Gamma}\left(\mathcal{W}_{h}\right)$. From $\operatorname{div}_{\Gamma} \mathrm{R}^{\Gamma} \boldsymbol{\mu}=\operatorname{div}_{\Gamma} \boldsymbol{\mu}$ we conclude that $\operatorname{div}_{\Gamma}\left(\mathrm{X}_{\Gamma}\left(\mathcal{W}_{h}\right)\right) \subset Q_{h}$. We see that $\mathrm{P}_{h}^{X}$ has to be applied to functions with $\operatorname{discrete} \operatorname{div}_{\Gamma}$ only. We remind of Lemma 16 , which bears out that $\mathrm{X}_{\Gamma}\left(\mathcal{W}_{h}\right)$ is contained in the domain of the local interpolation operators $\Pi_{h}$. We discover that a perfectly valid candidate for $\mathrm{P}_{h}^{X}$ is the local interpolation operator: $\mathrm{P}_{h}^{X}:=\Pi_{h}$. Then, Lemma 16 is the key to uniform convergence $\left(\mathrm{Id}-\mathrm{P}_{h}^{X}\right) \mathrm{X}_{\Gamma \mid \mathcal{W}_{h}} \rightarrow 0$.

Lemma 17. There is a $C_{*}=C_{*}(\Omega, p$, shape regularity $)>0$ such that for all $\boldsymbol{\mu}_{h} \in$ $\mathcal{W}_{h}$

$$
\left\|\left(\operatorname{Id}-\mathrm{P}_{h}^{X}\right) \mathrm{X}_{\Gamma} \boldsymbol{\mu}_{h}\right\|_{\boldsymbol{H}_{\times}\left(\operatorname{div}_{\Gamma}, \Gamma\right)} \leq C_{*} h^{1 / 2}\left\|\operatorname{div}_{\Gamma} \boldsymbol{\mu}_{h}\right\|_{H^{-\frac{1}{2}(\Gamma)}} .
$$

Proof. Note that $\left(\mathrm{Id}-\Pi_{h}\right) \mathrm{X}_{\Gamma} \boldsymbol{\mu}_{h}=\left(\mathrm{Id}-\Pi_{h}\right)\left(2 \mathrm{R}^{\Gamma}-\mathrm{Id}\right) \boldsymbol{\mu}_{h}=2\left(\mathrm{Id}-\Pi_{h}\right) \mathrm{R}^{\Gamma} \boldsymbol{\mu}_{h}$, and that $\operatorname{div}_{\Gamma} \mathrm{R}^{\Gamma} \boldsymbol{\mu}_{h}=\operatorname{div}_{\Gamma} \boldsymbol{\mu}_{h}$. Thus, for the estimate of the $\mathbf{L}_{\mathbf{t}}^{2}$ norm, we need only combine Lemma 16 (applied to $\mathrm{R}^{\Gamma} \boldsymbol{\mu}_{h}$ ) with (20). The observation, based on the commuting diagram property (57), that

$$
\operatorname{div}_{\Gamma}\left(\left(\operatorname{Id}-\Pi_{h}\right) \mathrm{X}_{\Gamma} \boldsymbol{\mu}_{h}\right)=\left(\mathrm{Id}-Q_{h}\right) \operatorname{div}_{\Gamma}\left(\mathrm{X}_{\Gamma} \boldsymbol{\mu}_{h}\right)=\left(\mathrm{Id}-Q_{h}\right) \operatorname{div}_{\Gamma} \boldsymbol{\mu}_{h}=0
$$

finishes the proof.

Using (66) and (67) in (65), we obtain:

$$
\left|a\left(\boldsymbol{\mu}_{h}, \boldsymbol{\eta}_{h}\right)\right| \geq\left(C_{G}-C_{A}\left(\epsilon(h)+C_{*} h^{\frac{1}{2}}\right)\right)\left\|\boldsymbol{\mu}_{h}\right\|_{\boldsymbol{H}_{\times}^{-\frac{1}{2}}\left(\operatorname{div}_{\Gamma}, \Gamma\right)}^{2} .
$$

This means that for $h$ small enough to ensure $1-C_{A}\left(\epsilon(h)+C_{*} h^{\frac{1}{2}}\right) / C_{G}>\frac{1}{2}$ we have the discrete inf-sup condition (62). This yields the main result:

Theorem 15. Provided that $\kappa^{2}$ is not an interior electric eigenvalue, there is a $h^{*}>0$, depending on the parameters of the continuous problem and the shaperegularity of the triangulation, such that a unique solution $\boldsymbol{\lambda}_{h} \in \mathcal{W}_{h}$ of the discretized problem (60) exists, provided that $h<h_{*}$. It supplies an asymptotically optimal approximation to the continuous solution $\boldsymbol{\lambda}$ of (60) in the sense of (63).

Exactly the same arguments apply to (48) and give us an analogue of Thm. 15 for the Galerkin BEM discretization in the case of the transmission problem. 
Remark 2. Asymptotic quasi-optimality alone does not provide information about the actual speed of convergence as $h \rightarrow 0$, unless we have information about the smoothness of $\boldsymbol{\lambda}$. To assess the regularity of $\boldsymbol{\lambda}$ it is necessary to recall its meaning as a boundary value or the jump of a trace of Maxwell solutions. Then the results on the regularity of Maxwell solutions given in [34] can be used. Ultimately we will always have $\boldsymbol{\lambda} \in \boldsymbol{H}_{\times}^{s}\left(\operatorname{div}_{\Gamma}, \Gamma\right)$ for some $s>0$ depending on the excitation and the geometry of $\Gamma$. In combination with Thm. 14 we can predict asymptotic rates of convergence for the $h$-version of the Galerkin boundary element schemes.

Remark 3. A striking difference between (42) and (49) is the choice of unknowns. In the indirect method $\lambda$ is a jump. Hence, when solving the boundary integral equations on a polyhedron, the unknown of the indirect method will be affected by the corner and edge singularities of both interior and exterior Maxwell solutions [34]. As any edge is re-entrant when seen from either $\Omega_{s}$ or $\Omega^{\prime}$, the jump $\left[\gamma_{N}\right]_{\Gamma}(\mathbf{e})$ will invariably possess a very low regularity. As a consequence, it might be much harder to approximate by boundary elements than the unknown of the direct method.

In terms of Galerkin discretization the CFIE from Sect. 7.2 poses an extra diffculty, because of the composition of the integral operator $\mathbf{C}_{\kappa}$ and the smoothing operator $\mathrm{M}$. The usual trick to avoid such operator products is to switch to a mixed formulation introducing the new unknown $\mathbf{p}:=\mathrm{M} \zeta$. If we use the particular smoothing operator from (54), we get $\mathbf{p} \in \boldsymbol{H}_{\times}\left(\operatorname{div}_{\Gamma}, \Gamma\right)$ and may simply incorporate (54) into the eventual mixed variational problem: find $\boldsymbol{\zeta} \in \boldsymbol{H}_{\times}^{-\frac{1}{2}}\left(\operatorname{div}_{\Gamma}, \Gamma\right)$, $\mathbf{p} \in \boldsymbol{H}_{\times}\left(\operatorname{div}_{\Gamma}, \Gamma\right)$ such that for all $\boldsymbol{\mu} \in \boldsymbol{H}_{\times}^{-\frac{1}{2}}\left(\operatorname{div}_{\Gamma}, \Gamma\right), \mathbf{q} \in \boldsymbol{H}_{\times}\left(\operatorname{div}_{\Gamma}, \Gamma\right)$,

$$
\begin{aligned}
-i \eta\left\langle\mathbf{S}_{\kappa} \boldsymbol{\zeta}, \boldsymbol{\mu}\right\rangle_{\boldsymbol{\tau}, \Gamma}+\left\langle\left(\frac{1}{2} \operatorname{Id}-\mathbf{C}_{\kappa}\right) \mathbf{p}, \boldsymbol{\mu}\right\rangle_{\boldsymbol{\tau}, \Gamma} & =\left\langle\gamma_{\mathbf{t}}^{+} \mathbf{e}_{i}, \boldsymbol{\mu}\right\rangle_{\boldsymbol{\tau}, \Gamma}, \\
\langle\mathbf{q}, \boldsymbol{\zeta}\rangle_{\boldsymbol{\tau}, \Gamma}-\langle\mathbf{p}, \mathbf{q}\rangle_{0 ; \Gamma}-\left\langle\operatorname{div}_{\Gamma} \mathbf{p}, \operatorname{div}_{\Gamma} \mathbf{q}\right\rangle_{0 ; \Gamma} & =0 .
\end{aligned}
$$

Thanks to the compact embedding $\boldsymbol{H}_{\times}\left(\operatorname{div}_{\Gamma}, \Gamma\right) \hookrightarrow \boldsymbol{H}_{\times}^{-\frac{1}{2}}\left(\operatorname{div}_{\Gamma}, \Gamma\right)$ the offdiagonal terms in (68) are compact. Thus, a generalized Gårding inequality is immediate from Lemma 10. As far as the analysis of the Galerkin discretization in $\mathcal{W}_{h} \times \mathcal{W}_{h}$ is concerned, we only need to deal with the diagonal terms in (68): using exactly the same arguments as above we conclude the quasi-optimality of Galerkin solutions on sufficiently fine meshes. Please note that the estimates now employ the norm of the product space $\boldsymbol{H}_{\times}^{-\frac{1}{2}}\left(\operatorname{div}_{\Gamma}, \Gamma\right) \times \boldsymbol{H}_{\times}\left(\operatorname{div}_{\Gamma}, \Gamma\right)$. Thus, asymptotic rates of convergence will depend on the smoothness of both $\zeta$ and $\mathbf{p}$.

\subsection{Integral equations of the second kind}

Pitfalls have to be avoided when performing a Galerkin boundary element discretization of the Fredholm integral equation of the second kind (44). A straightforward Galerkin discretization would lead to: seek $\boldsymbol{\lambda}_{h} \in \mathcal{W}_{h}$ such that

$$
\left\langle\left(\frac{1}{2} \operatorname{Id}+\mathbf{C}_{\kappa}\right) \boldsymbol{\lambda}_{h}, \boldsymbol{\mu}_{h}\right\rangle_{\boldsymbol{\tau}, \Gamma}=\text { r.h.s. }\left(\boldsymbol{\mu}_{h}\right) \quad \forall \boldsymbol{\mu}_{h} \in \mathcal{W}_{h} .
$$


If $\Gamma \in C^{\infty}$, then existence and uniqueness of solutions of the continuous variational problem are clear from Theorem 11 . However, this does not necessarily remain true for (69). The cause of the difficulties is the failure of Theorem 2 to hold in the discrete setting. In other words, $\mathcal{W}_{h}$ may not be dual to itself with respect to the pairing $\langle\cdot, \cdot\rangle_{\boldsymbol{\tau}, \Gamma}$. More precisely, in [29, Section 3.1] it has been shown by means of Hodge decompositions that for $\mathrm{RT}_{0}$ boundary elements and quasiuniform families of surface meshes $\Gamma_{h}$ there is $\alpha>0$ and spaces $\mathcal{K}_{h} \subset \mathcal{W}_{h}$ such that, for all $h \in \mathbb{H}$, $\operatorname{dim} \mathcal{K}_{h} \geq \alpha \operatorname{dim} \mathcal{W}_{h}$ and

$$
\forall \boldsymbol{\mu}_{h} \in \mathcal{K}_{h} \sup _{\boldsymbol{\xi}_{h} \in \mathcal{W}_{h}} \frac{\left|\left\langle\boldsymbol{\mu}_{h}, \boldsymbol{\xi}_{h}\right\rangle_{\boldsymbol{\tau}, \Gamma}\right|}{\left\|\boldsymbol{\xi}_{h}\right\|_{\boldsymbol{H}_{\times}^{-\frac{1}{2}}\left(\operatorname{div}_{\Gamma}, \Gamma\right)}} \leq C h^{\frac{1}{2}}\left\|\boldsymbol{\mu}_{h}\right\|_{\boldsymbol{H}_{\times}^{-\frac{1}{2}}\left(\operatorname{div}_{\Gamma}, \Gamma\right)} .
$$

The discretization of $\langle\cdot, \cdot\rangle_{\boldsymbol{\tau}, \Gamma}$ on $\mathcal{W}_{h}$ is not stable! This bars us from deriving a discrete inf-sup condition, though the continuous bilinear form satisfies a generalized Gårding inequality.

Remark 4 . The instability of $\langle\cdot, \cdot\rangle_{\tau, \Gamma}$ in $\mathcal{W}$ also thwarts the straightforward application of an otherwise effective preconditioning strategy for boundary integral equations of the first kind, which is based on Calderón projectors [59]. The gist of the remedy, devised in [28], is to express $\langle\cdot, \cdot\rangle_{\boldsymbol{\tau}, \Gamma}$ via an approximate discrete Hodge decomposition of $\mathcal{W}$.

This instability forces us to switch from (44) to (45), before a Galerkin discretization by means of $\mathcal{W}_{h}$ becomes feasible: the stability of the $\boldsymbol{T} \boldsymbol{H}\left(\operatorname{div}_{\Gamma}, \Gamma\right)$ inner product in the discrete setting is a moot point. Hence, provided that the assumptions of Theorem 11 hold, the Galerkin discretization in $\mathcal{W}_{h}$ will produce asymptotically optimally convergent solutions on sufficiently fine meshes. The proof follows the standard approach to coercive variational problems [62]. However, note that all estimates will be based on the $\boldsymbol{H}_{\times}\left(\operatorname{div}_{\Gamma}, \Gamma\right)$-norm, that is

$$
\left\|\boldsymbol{\lambda}-\boldsymbol{\lambda}_{h}\right\|_{\boldsymbol{H}_{\times}\left(\operatorname{div}_{\Gamma}, \Gamma\right)} \leq C \inf _{\boldsymbol{\eta} \in \mathcal{W}_{h}}\left\|\boldsymbol{\lambda}-\boldsymbol{\eta}_{h}\right\|_{\boldsymbol{H}_{\times}\left(\operatorname{div}_{\Gamma}, \Gamma\right)} .
$$

We point out that the bilinear expressions $\left(\boldsymbol{\lambda}_{h}, \boldsymbol{\mu}_{h}\right) \mapsto\left\langle\operatorname{div}_{\Gamma} \mathbf{C}_{\kappa} \boldsymbol{\lambda}_{h}, \operatorname{div}_{\Gamma} \boldsymbol{\mu}_{h}\right\rangle_{0 ; \Gamma}$ that have to be evaluated for basis functions of $\mathcal{W}_{h}$ in order to get the system matrix can be converted into sums of two integrals over $\Gamma \times \Gamma$ featuring weakly singular kernels. Details can be found in the proof of Lemma 11.

\section{Coupling of Finite Elements and Boundary Elements}

The solution of the transmission problem of electromagnetic scattering by means of direct boundary integral equations is confined to the case of homogeneous scatterers, because the simple representation formula (26) for Maxwell solutions cannot accommodate variable material coefficients $\underline{\epsilon}=\underline{\epsilon}(\mathbf{x}), \underline{\mu}=\underline{\mu}(\mathbf{x}), \mathbf{x} \in \Omega_{s}$. This situation poses no problems for a Galerkin finite element discretization of the spatial 
variational problem inside $\Omega_{s}$. On the other hand, the field problem in the air region $\Omega^{\prime}$ is not amenable to a treatment by classical finite elements, but offers a perfect setting for the boundary element methods discussed in the previous sections. Thus, it is natural to tackle scattering at an inhomogeneous body by a combined Galerkin discretization involving both finite elements and boundary elements. In this section the focus will be on a method based on the Calderon projector $\mathbb{P}_{\kappa}^{+}$from (34).

Using $a_{M}(\cdot, \cdot)$ defined in (15), the electric field in $\Omega_{s}$ satisfies

$$
a_{M}(\mathbf{e}, \mathbf{v})-\left\langle\underline{\boldsymbol{\mu}}^{-1} \gamma_{\mathbf{t}}^{-} \operatorname{curl} \mathbf{e}, \gamma_{\mathbf{t}}^{-} \mathbf{v}\right\rangle_{\boldsymbol{\tau}, \Gamma}=0
$$

for all $\mathbf{v} \in \boldsymbol{H}\left(\mathbf{c u r l} ; \Omega_{s}\right)$. The gist of coupling is to employ an operator representation of the Dirichlet-to-Neumann map $\operatorname{DtN}_{\kappa}^{+}: \boldsymbol{H}_{\times}^{-\frac{1}{2}}\left(\operatorname{div}_{\Gamma}, \Gamma\right) \mapsto \boldsymbol{H}_{\times}^{-\frac{1}{2}}\left(\operatorname{div}_{\Gamma}, \Gamma\right)$, which is a linear operator returning $\gamma_{N}^{+} \mathbf{e}$ for a Maxwell solution e in $\Omega^{\prime}$ if $\gamma_{\mathbf{t}}^{+} \mathbf{e}$ is prescribed. If this was available, we could use the transmission conditions

$$
\gamma_{\mathbf{t}}^{-} \mathbf{e}=\gamma_{\mathbf{t}}^{+} \mathbf{e}+\gamma_{\mathbf{t}}^{+} \mathbf{e}_{i} \quad, \quad \underline{\boldsymbol{\mu}}^{-1} \gamma_{\mathbf{t}}^{-} \operatorname{curl} \mathbf{e}=\frac{\kappa}{\mu_{0}} \gamma_{N}^{+} \mathbf{e}+\gamma_{\mathbf{t}}^{+} \mathbf{h}_{i},
$$

to cast the scattering problem in the variational form: seek e $\in \boldsymbol{H}\left(\mathbf{c u r l} ; \Omega_{s}\right)$ such that for all $\mathbf{v} \in \boldsymbol{H}\left(\mathbf{c u r l} ; \Omega_{s}\right)$

$$
a_{M}(\mathbf{e}, \mathbf{v})-\kappa \mu_{0}^{-1}\left\langle\operatorname{DtN}_{\kappa}^{+} \gamma_{\mathbf{t}}^{-} \mathbf{e}, \gamma_{\mathbf{t}}^{-} \mathbf{v}\right\rangle_{\boldsymbol{\tau}, \Gamma}=\operatorname{r.h} . \mathrm{s}(\mathbf{v})
$$

By Thm. 8 either row of the interior Calderon projector $\mathbb{P}_{\kappa}^{-}$immediately supplies a realization of $\mathrm{DtN}_{\kappa}^{+}$:

$$
\operatorname{DtN}_{\kappa}^{+}=-\left(\frac{1}{2} \mathrm{Id}+\mathbf{C}_{\kappa}\right)^{-1} \mathbf{S}_{\kappa} \quad, \quad \operatorname{DtN}_{\kappa}^{+}=-\mathbf{S}_{\kappa}^{-1}\left(\frac{1}{2} \mathrm{Id}+\mathbf{C}_{\kappa}\right) .
$$

Both formulae describe the same operator, but appear vastly different. The reason is that they both break the inherent symmetry of magnetic and electric fields. Symmetry can be preserved by combining both rows of $\mathbb{P}_{\kappa}^{+}$in a clever manner:

$$
\operatorname{DtN}_{\kappa}^{+}=-\mathbf{S}_{\kappa}-\left(\frac{1}{2} \mathrm{Id}-\mathbf{C}_{\kappa}\right) \mathbf{S}_{\kappa}^{-1}\left(\frac{1}{2} \operatorname{Id}+\mathbf{C}_{\kappa}\right) .
$$

This discovery was first presented in [32] and is the foundation for the so-called symmetric approach to marrying finite elements and boundary elements. It has been applied to a wide range of transmission problems, see, for instance $[22,23,50]$. In the case of electromagnetism the idea was examined theoretically in [4-6], and in [9] for a related problem involving impedance boundary conditions.

Of course, a variational formulation suited for Galerkin discretization has to dispense with the explicit inverse $\mathbf{S}_{\kappa}^{-1}$. Instead another equation is added, which leads to: seek e $\in \boldsymbol{H}\left(\mathbf{c u r l} ; \Omega_{s}\right), \boldsymbol{\lambda} \in \boldsymbol{H}_{\times}^{-\frac{1}{2}}\left(\operatorname{div}_{\Gamma}, \Gamma\right)$ with

$$
\begin{aligned}
a_{M}\left(\mathbf{e}, \mathbf{e}^{\prime}\right)+\left\langle\frac{\kappa}{\mu_{0}} \mathbf{S}_{\kappa} \gamma_{\mathbf{t}}^{-} \mathbf{e}, \gamma_{\mathbf{t}}^{-} \mathbf{e}^{\prime}\right\rangle_{\boldsymbol{\tau}, \Gamma}-\left\langle\frac{\kappa}{\mu_{0}}\left(\frac{1}{2} \operatorname{Id}-\mathbf{C}_{\kappa}\right) \boldsymbol{\lambda}, \gamma_{\mathbf{t}}^{-} \mathbf{e}^{\prime}\right\rangle_{\boldsymbol{\tau}, \Gamma} & =\ldots, \\
\left\langle\left(\frac{1}{2} \operatorname{Id}+\mathbf{C}_{\kappa}\right) \gamma_{\mathbf{t}}^{-} \mathbf{e}, \boldsymbol{\lambda}^{\prime}\right\rangle_{\boldsymbol{\tau}, \Gamma}+ & =\ldots,
\end{aligned}
$$


for all $\mathbf{e}^{\prime} \in \boldsymbol{H}\left(\operatorname{curl} ; \Omega_{s}\right), \boldsymbol{\lambda}^{\prime} \in \boldsymbol{H}_{\times}^{-\frac{1}{2}}\left(\operatorname{div}_{\Gamma}, \Gamma\right)$. The new unknown $\boldsymbol{\lambda}$ will provide the exterior Neumann trace $\gamma_{N}^{+} \mathbf{e}$.

Note that the symmetric version of $\mathrm{DtN}_{\kappa}^{+}$involves the inverse of $\mathbf{S}_{\kappa}$. This suggests that "forbidden wave numbers" will also haunt the coupled formulations, $c f$. Sect. 7. Similar to Lemma 14 one proves the following theorem, see [44].

Theorem 16. If $\kappa^{2}$ is not an interior electric eigenvalue, a solution $(\mathbf{e}, \boldsymbol{\lambda})$ of (74) provides a solution of the transmission problem (1) by retaining e in $\Omega_{s}$ and using the exterior Stratton-Chu representation formula (26) with the data $\left(\gamma_{\mathbf{t}}^{-} \mathbf{e}-\gamma_{\mathbf{t}}^{+} \mathbf{e}_{i}, \boldsymbol{\lambda}\right)$.

Corollary 6. If $\kappa^{2}$ is not an interior electric eigenvalue, the solution $(\mathbf{e}, \boldsymbol{\lambda})$ of (74) is unique.

We point out that even if $\kappa$ violates the assumption of the theorem, the solution for e will remain unique. This will no longer be true for $\boldsymbol{\lambda}$, which is unique only up to Neumann traces of interior electric eigenmodes. This can be seen by refining the arguments in the proof of Theorem 16.

We denote by $\mathbf{d}_{\kappa}$ the bilinear form on $\boldsymbol{H}\left(\operatorname{curl} ; \Omega_{s}\right) \times \boldsymbol{H}_{\times}^{-\frac{1}{2}}\left(\operatorname{div}_{\Gamma}, \Gamma\right)$ that is associated with the the variational problem (74). Pursuing the same policy as in Sect. 3 and 7, we aim to establish a generalized Gå rding inequality for $\mathbf{d}_{\kappa}$. Of course, the splitting idea will pave the way. More precisely, the crucial "sign flipping isomorphism" $\mathbb{X}_{\mathcal{V}}$ will involve both splittings (18) and (21) employed in Sects. 3 and 7. Writing, $\mathcal{V}:=\boldsymbol{H}\left(\mathbf{c u r l} ; \Omega_{s}\right) \times \boldsymbol{H}_{\times}^{-\frac{1}{2}}\left(\operatorname{div}_{\Gamma}, \Gamma\right)$, it reads

$$
\mathbb{X}_{\mathcal{V}}\left(\begin{array}{l}
\mathbf{u} \\
\boldsymbol{\xi}
\end{array}\right):=\left(\begin{array}{c}
(\mathrm{R}-\mathrm{Z}) \mathbf{u} \\
\left(\mathrm{R}^{\Gamma}-\mathrm{Z}^{\Gamma}\right) \boldsymbol{\xi}
\end{array}\right): \mathcal{V} \mapsto \mathcal{V}
$$

We make the important observation that the trace $\gamma_{\mathbf{t}}^{-}$maps curl-free vectorfields into $\mathcal{N}(\Gamma)$. In addition we can use the symmetry of $\mathbf{C}_{\kappa}$ stated in lemma 6 and proceed as in the proof of Thm. 9. This will give us the desired strengthened Gå rding inequality:

Theorem 17. There exists a compact bilinear form $\mathbf{c}: \mathcal{V} \times \mathcal{V} \mapsto \mathbb{C}$ and a constant $C_{G}>0$ such that

$$
\begin{aligned}
\left|\mathbf{d}_{\kappa}\left(\left(\begin{array}{l}
\mathbf{u} \\
\boldsymbol{\mu}
\end{array}\right), \mathbb{X}_{\mathcal{V}}\left(\begin{array}{l}
\overline{\mathbf{u}} \\
\overline{\boldsymbol{\mu}}
\end{array}\right)\right)-\boldsymbol{c}\left(\left(\begin{array}{c}
\mathbf{u} \\
\boldsymbol{\mu}
\end{array}\right),\left(\begin{array}{l}
\overline{\mathbf{u}} \\
\overline{\boldsymbol{\mu}}
\end{array}\right)\right)\right| & \\
& \geq C_{G}\left(\|\mathbf{u}\|_{\boldsymbol{H}\left(\mathbf{c u r l} ; \Omega_{s}\right)}^{2}+\|\boldsymbol{\mu}\|_{\boldsymbol{H}_{\times}^{-\frac{1}{2}}\left(\operatorname{div}_{\Gamma}, \Gamma\right)}^{2}\right)
\end{aligned}
$$

holds for all $\mathbf{u} \in \boldsymbol{H}\left(\mathbf{c u r l} ; \Omega_{s}\right), \boldsymbol{\mu} \in \boldsymbol{H}_{\times}^{-\frac{1}{2}}\left(\operatorname{div}_{\Gamma}, \Gamma\right)$.

Hence, in conjuction with Cor. 6, a Fredholm alternative argument confirms the existence of solutions of the variational problem (74). 
Besides $\Gamma_{h}$ the Galerkin discretization of (74) requires a triangulation $\Omega_{h}$ of $\Omega_{s}$. In principle, both can be independent of each other, but implementation is greatly facilitated if $\Gamma_{h}=\Omega_{h \mid \Gamma}$. Then, we can rely on the $\boldsymbol{H}_{\times}\left(\operatorname{div}_{\Gamma}, \Gamma\right)$-conforming boundary element spaces $\mathcal{W}_{h}$ to approximate $\boldsymbol{\lambda}$, and special $\boldsymbol{H}\left(\mathbf{c u r l} ; \Omega_{s}\right)$-conforming finite elements for e. The latter are thoroughly discussed in [45, Ch. 3]. They enjoy all the properties that permit us to prove a discrete inf-sup-condition as in Sect. 9.1. Thus we can get asymptotic quasi-optimality of discrete solutions obtained by the symmetric coupling of finite elements and boundary elements for the electromagnetic scattering problem.

\section{References}

1. R. AdAms, Sobolev Spaces, Academic Press, New York, 1975.

2. A. Alonso AND A. VALli, Some remarks on the characterization of the space of tangential traces of $H(\operatorname{rot} ; \Omega)$ and the construction of an extension operator, Manuscripta mathematica, 89 (1996), pp. 159-178.

3. H. AMmARI AND S. HE, Effective impedance boundary conditions for an inhomogeneous thin layer on a curved metallic surface, IEEE Trans. Antennas and Propagation, 46 (1998), pp. 710-715.

4. H. AMmARI AND J. NÉDÉLEC, Couplage éléments finis-équations intégrales pour la résolution des équations de Maxwell en milieu hétérogene, in Equations aux derivees partielles et applications. Articles dedies a Jacques-Louis Lions, Gauthier-Villars, Paris, 1998, pp. 19-33.

5. H. AMMARI AND J.-C. NÉDÉLEC, Coupling of finite and boundary element methods for the time-harmonic Maxwell equations. II: A symmetric formulation, in The Maz'ya anniversary collection. Vol. 2, J. Rossmann, ed., vol. 110 of Oper. Theory, Adv. Appl., Birkhäuser, Basel, 1999, pp. 23-32.

6. - Coupling integral equations method and finite volume elements for the resolution of the Leontovich boundary value problem for the time-harmonic Maxwell equations in three dimensional heterogeneous media, in Mathematical aspects of boundary element methods. Minisymposium during the IABEM 98 conference, dedicated to Vladimir Maz'ya on the occasion of his 60th birthday on 31st December 1997, M. Bonnet, ed., vol. 41 of CRC Research Notes in Mathematics, CRC Press, Boca Raton, FL, 2000, pp. 11-22.

7. C. Amrouche, C. Bernardi, M. Dauge, and V. Girault, Vector potentials in three-dimensional nonsmooth domains, Math. Meth. Appl. Sci., 21 (1998), pp. 823-864.

8. I. BАВUŠKA, Error bounds for the finite element method, Numer. Math., 16 (1971), pp. 322-333.

9. A. BENDALI, Boundary element solution of scattering problems relative to a generalized impedance boundary condition, in Partial differential equations: Theory and numerical solution. Proceedings of the ICM'98 satellite conference, Prague, Czech Republic, August 10-16, 1998., W. Jäger, ed., vol. 406 of CRC Res. Notes Math., Boca Raton, FL, 2000, Chapman \& Hall/CRC, pp. 10-24.

10. A. BENDALI AND L. Vernhet, The Leontovich bondary value problem and its boundary integral equations solution, Preprint, CNRS-UPS-INSA, Department de Genie Mathematique, Toulouse, France, 2001.

11. H. BreZIS, Analyse fonctionnelle. Théorie et applications, Masson, Paris, 1983. 
12. F. BRezzi, J. Douglas, AND D. Marini, Two families of mixed finite elements for 2nd order elliptic problems, Numer. Math., 47 (1985), pp. 217-235.

13. F. BREZZI AND M. ForTIN, Mixed and hybrid finite element methods, Springer, 1991.

14. A. BUFFA, Hodge decompositions on the boundary of a polyhedron: The multiconnected case, Math. Mod. Meth. Appl. Sci., 11 (2001), pp. 1491-1504.

15. — Traces theorems for functional spaces related to Maxwell equations: An overwiew. To appear in Proceedings of the GAMM Workshop on Computational Electromagnetics, Kiel, January 26th - 28th, 2001.

16. A. Buffa AND S. Christiansen, The electric field integral equation on Lipschitz screens: Definition and numerical approximation, Numer. Mathem. (2002), DOI 10.1007/s00211-002-0422-0.

17. A. BUfFA AND P. CiARLET, JR., On traces for functional spaces related to Maxwell's equations. Part I: An integration by parts formula in Lipschitz polyhedra., Math. Meth. Appl. Sci., 24 (2001), pp. 9-30.

18. - On traces for functional spaces related to Maxwell's equations. Part II: Hodge decompositions on the boundary of Lipschitz polyhedra and applications, Math. Meth. Appl. Sci., 21 (2001), pp. 31-48.

19. A. Buffa, M. CostaBel, AND C. Schwab, Boundary element methods for Maxwell's equations on non-smooth domains, Numer. Mathem. 92 (2002) 4, pp. 679-710.

20. A. Buffa, M. Costabel, And D. Sheen, On traces for $\mathbf{H}(\mathbf{c u r l}, \Omega)$ in Lipschitz domains, J. Math. Anal. Appl., 276/2 (2002), pp. 845-876.

21. A. Buffa, R. Hiptmair, T. von PetersdorfF, AND C. SChwab, Boundary element methods for Maxwell equations on Lipschitz domains, Numer. Math., (2002). To appear.

22. C. CARSTENSEN, A posteriori error estimate for the symmetric coupling of finite elements and boundary elements, Computing, 57 (1996), pp. 301-322.

23. C. CARSTENSEN AND P. WRIGGERS, On the symmetric boundary element method and the symmetric coupling of boundary elements and finite elements, IMA J. Numer. Anal., 17 (1997), pp. 201-238.

24. M. Cessent, Mathematical Methods in Electromagnetism, vol. 41 of Advances in Mathematics for Applied Sciences, World Scientific, Singapore, 1996.

25. G. Chen AND J. ZHOU, Boundary Element Methods, Academic Press, New York, 1992.

26. S. CHRISTIANSEN, Discrete Fredholm properties and convergence estimates for the EFIE, Technical Report 453, CMAP, Ecole Polytechique, Paris, France, 2000.

27. - Mixed boundary element method for eddy current problems, Research Report 2002-16, SAM, ETH Zürich, Zürich, Switzerland, 2002.

28. S. ChristiAnSEN AND J.-C. NÉDÉLEC, Des prćonditionneurs pour la résolution numérique des équations intégrales de frontiére de l'electromagnétisme, C.R. Acad. Sci. Paris, Ser. I Math, 31 (2000), pp. 617-622.

29. - A preconditioner for the electric field integral equation based on Calderón formulas. To appear in SIAM J. Numer. Anal.

30. P. CiARLET, The Finite Element Method for Elliptic Problems, vol. 4 of Studies in Mathematics and its Applications, North-Holland, Amsterdam, 1978.

31. D. Colton And R. KRess, Inverse Acoustic and Electromagnetic Scattering Theory, vol. 93 of Applied Mathematical Sciences, Springer, Heidelberg, 2nd ed., 1998.

32. M. COSTABEL, Symmetric methods for the coupling of finite elements and boundary elements, in Boundary Elements IX, C. Brebbia, W. Wendland, and G. Kuhn, eds., Springer, Berlin, 1987, pp. 411-420.

33. - Boundary integral operators on Lipschitz domains: Elementary results, SIAM J. Math. Anal., 19 (1988), pp. 613-626. 
34. M. Costabel And M. Dauge, Singularities of Maxwell's equations on polyhedral domains, in Analysis, Numerics and Applications of Differential and Integral Equations, M. Bach, ed., vol. 379 of Longman Pitman Res. Notes Math. Ser., Addison Wesley, Harlow, 1998, pp. 69-76.

35. — Maxwell and Lamé eigenvalues on polyhedra, Math. Methods Appl. Sci., 22 (1999), pp. 243-258.

36. R. Dautray And J.-L. Lions, Mathematical Analysis and Numerical Methods for Science and Technology, vol. 4, Springer, Berlin, 1990.

37. A. DE LA BourdonNAYE, Some formulations coupling finite element amd integral equation method for Helmholtz equation and electromagnetism, Numer. Math., 69 (1995), pp. 257-268.

38. L. DemKowICZ, Asymptotic convergence in finite and boundary element methods: Part 1, Theoretical results, Comput. Math. Appl., 27 (1994), pp. 69-84.

39. V. GiRAUlt AND P. RAVIART, Finite element methods for Navier-Stokes equations, Springer, Berlin, 1986.

40. P. GRISVARD, Elliptic Problems in Nonsmooth Domains, Pitman, Boston, 1985.

41. - Singularities in boundary value problems, vol. 22 of Research Notes in Applied Mathematics, Springer, New York, 1992.

42. W. HACKBUSCH, Integral equations. Theory and numerical treatment., vol. 120 of International Series of Numerical Mathematics, Birkhäuser, Basel, 1995.

43. C. HAZARD AND M. LENOIR, On the solution of time-harmonic scattering problems for Maxwell's equations, SIAM J. Math. Anal., 27 (1996), pp. 1597-1630.

44. R. Hiptmair, Coupling of finite elements and boundary elements in electromagnetic scattering, Report 164, SFB 382, Universität Tübingen, Tübingen, Germany, July 2001. Submitted to SIAM J. Numer. Anal.

45. - Finite elements in computational electromagnetism, Acta Numerica, (2002), pp. 237-339.

46. - Symmetric coupling for eddy current problems, SIAM J. Numer. Anal., 40 (2002), pp. 41-65.

47. R. Hiptmair AND C. SCHWAB, Natural boundary element methods for the electric field integral equation on polyhedra, SIAM J. Numer. Anal., 40 (2002), pp. 66-86.

48. R. KRESS, On the boundary operator in electromagnetic scattering, Proc. Royal Soc. Edinburgh, 103A (1986), pp. 91-98.

49. - Linear Integral Equations, vol. 82 of Applied Mathematical Sciences, Springer, Berlin, 1989.

50. M. KUHN AND O. STEINBACH, FEM-BEM coupling for $3 d$ exterior magnetic field problems, Math. Meth. Appl. Sci., (2002). To appear.

51. R. MCCAMY AND E. Stephan, Solution procedures for three-dimensional eddycurrent problems, J. Math. Anal. Appl., 101 (1984), pp. 348-379.

52. W. MCLEAN, Strongly Elliptic Systems and Boundary Integral Equations, Cambridge University Press, Cambridge, UK, 2000.

53. J.-C. NÉDÉLEC, Acoustic and Electromagnetic Equations: Integral Representations for Harmonic Problems, vol. 44 of Applied Mathematical Sciences, Springer, Berlin, 2001.

54. A. Nethe, R.QuAst, AND H. STAhLmann, Boundary conditions for high frequency eddy current problems, IEEE Trans. Mag., 34 (1998), pp. 3331-3334.

55. L. PAQUet, Problemes mixtes pour le systeme de Maxwell, Ann. Fac. Sci. Toulouse, V. Ser., 4 (1982), pp. 103-141.

56. P. A. Raviart And J. M. Thomas, A Mixed Finite Element Method for Second Order Elliptic Problems, vol. 606 of Springer Lecture Notes in Mathematics, Springer, Ney York, 1977, pp. 292-315. 
57. M. REISSEL, On a transmission boundary-value problem for the time-harmonic Maxwell equations without displacement currents, SIAM J. Math. Anal., 24 (1993), pp. $1440-1457$.

58. A. SCHATZ, An observation concerning Ritz-Galerkin methods with indefinite bilinear forms, Math. Comp., 28 (1974), pp. 959-962.

59. O. STEINBACH AND W. WENDLAND, The construction of some efficient preconditioners in the boundary element method, Adv. Comput. Math., 9 (1998), pp. 191-216.

60. J. StRATtON AND L. Chu, Diffraction theory of electromagnetic waves, Phys. Rev., 56 (1939), pp. 99-107.

61. T. VON PETERSDORFF, Boundary integral equations for mixed Dirichlet, Neumann and transmission problems, Math. Meth. Appl. Sci., 11 (1989), pp. 185-213.

62. W. WENDLAND, Boundary element methods for elliptic problems, in Mathematical Theory of Finite and Boundary Element Methods, A. Schatz, V. Thomée, and W. Wendland, eds., vol. 15 of DMV-Seminar, Birkhäuser, Basel, 1990, pp. 219-276.

63. J. XU AND L. ZIKATANOv, Some oberservations on Babuška and Brezzi theories, Report AM222, PennState Department of Mathematics, College Park, PA, September 2000. To appear in Numer. Math.

\section{A Scattering from coated dielectric objects}

Now, we consider a partition of $\Gamma:=\partial \Omega_{s}$ into a part $\Gamma_{\mathrm{PEC}}$ covered by a perfectly conducting coating, and a part $\Gamma_{o}$ that can be penetrated by electromagnetic fields. Further, we assume that $\Omega_{s}$ is filled with an isotropic, homogeneous dielectric material with material parameters $\epsilon^{-}$and $\mu^{-}$. Hence we end up with a wave number $\kappa^{-}:=\omega \sqrt{\epsilon^{-} \mu^{-}}$inside $\Omega_{s}$, whereas $\kappa^{+}:=\omega \sqrt{\epsilon_{0} \mu_{0}}$ has to be used in $\Omega^{\prime}$. On $\Gamma$ we find the following boundary and transmission conditions:

$$
\begin{gathered}
\gamma_{\mathbf{t}}^{-} \mathbf{e}=\gamma_{\mathbf{t}}^{+} \mathbf{e}=0 \quad \text { on } \Gamma_{\mathrm{PEC}} \quad, \quad \gamma_{\mathbf{t}}^{-} \mathbf{e}=\gamma_{\mathbf{t}}^{+} \mathbf{e} \quad \text { on } \Gamma_{o}, \\
\frac{\kappa^{-}}{\mu^{-}} \gamma_{N}^{-} \mathbf{e}=\frac{\kappa^{+}}{\mu_{0}} \gamma_{N}^{+} \mathbf{e} \quad \text { on } \Gamma_{o} .
\end{gathered}
$$

We aim to establish a variational direct boundary integral equation formulation for this transmission problem. We closely follow the approach in Sect. 7.1, p. 28, and will reuse the notations introduced there.

First, we recall some results about traces of functions in $\boldsymbol{H}(\mathbf{c u r l} ; \Omega)$ onto parts of the boundary, $c f$. [18]. To this end, let $\Omega \subset \mathbb{R}^{3}$ be a generic domain and $\Gamma_{o} \subset \partial \Omega$ an open subset of its piecewise smooth Lipschitz boundary $\Gamma$. We take for granted that $\Gamma_{o}$ is a union of faces of $\Gamma$. We introduce the space

$$
\boldsymbol{H}_{\Gamma \backslash \Gamma_{o}}(\operatorname{curl} ; \Omega):=\left\{\mathbf{u} \in \boldsymbol{H}(\operatorname{curl} ; \Omega), \gamma_{\mathbf{t}} \mathbf{u}=0 \text { on } \Gamma \backslash \Gamma_{o}\right\},
$$

and the two trace spaces

$$
\begin{aligned}
\boldsymbol{H}_{\times}^{-\frac{1}{2}}\left(\operatorname{div}_{\Gamma}, \Gamma_{o}\right) & :=\left\{\boldsymbol{\mu} \in \boldsymbol{H}_{\times}^{-\frac{1}{2}}\left(\Gamma_{o}\right), \operatorname{div}_{\Gamma} \boldsymbol{\mu} \in H^{-\frac{1}{2}}\left(\Gamma_{o}\right)\right\} \\
\boldsymbol{H}_{\times, 00}^{-\frac{1}{2}}\left(\operatorname{div}_{\Gamma}, \Gamma_{o}\right) & :=\left\{\boldsymbol{\mu} \in \boldsymbol{H}_{\times, 00}^{-\frac{1}{2}}\left(\Gamma_{o}\right), \operatorname{div}_{\Gamma} \boldsymbol{\mu} \in H_{00}^{-\frac{1}{2}}\left(\Gamma_{o}\right)\right\}
\end{aligned}
$$

From [18, Thm. 5.3] we get the following fundamental trace theorem. Its statement makes use of the restriction operator $r_{o} \boldsymbol{\mu}:=\boldsymbol{\mu}_{\mid \Gamma_{o}}$ in the sense of distributions. 
Theorem 18. The restricted trace mapping $r_{o} \circ \gamma_{\mathbf{t}}: \boldsymbol{H}(\operatorname{curl} ; \Omega) \mapsto \boldsymbol{H}_{\times, 00}^{-\frac{1}{2}}\left(\operatorname{div}_{\Gamma}, \Gamma_{o}\right)$ is continuous and surjective.

The trace mapping $\gamma_{\mathbf{t}}: \boldsymbol{H}_{\Gamma \backslash \Gamma_{o}}(\operatorname{curl} ; \Omega) \mapsto \boldsymbol{H}_{\times}^{-\frac{1}{2}}\left(\operatorname{div}_{\Gamma}, \Gamma_{o}\right)$ is continuous and surjective.

We immediately infer that $\boldsymbol{H}_{\times}^{-\frac{1}{2}}\left(\operatorname{div}_{\Gamma}, \Gamma_{o}\right)$ is the space of tangential vectorfields on $\Gamma_{o}$ that yield a function in $\boldsymbol{H}_{\times}^{-\frac{1}{2}}\left(\operatorname{div}_{\Gamma}, \Gamma\right)$ when extended by zero to $\Gamma$.

The duality for the partial trace spaces is explained in $[18$, Sect. 5].

Theorem 19. The spaces $\boldsymbol{H}_{\times}^{-\frac{1}{2}}\left(\operatorname{div}_{\Gamma}, \Gamma_{o}\right)$ and $\boldsymbol{H}_{\times, 00}^{-\frac{1}{2}}\left(\operatorname{div}_{\Gamma}, \Gamma_{o}\right)$ are dual to each other with respect to the pairing $\langle\cdot, \cdot\rangle_{\boldsymbol{\tau}, \Gamma}$.

As in Sect. 7.1 we introduce the scaled traces

$$
\left(\boldsymbol{\zeta}^{+}, \boldsymbol{\lambda}^{+}\right)=\left(\gamma_{\mathbf{t}}^{+} \mathbf{e}, \frac{\kappa^{+}}{\mu_{0}} \gamma_{N}^{+} \mathbf{e}\right) \quad, \quad\left(\boldsymbol{\zeta}^{-}, \boldsymbol{\lambda}^{-}\right)=\left(\gamma_{\mathbf{t}}^{-} \mathbf{e}, \frac{\kappa^{-}}{\mu^{-}} \gamma_{N}^{-} \mathbf{e}\right) .
$$

With this notation the transmission conditions on $\Gamma_{o}$ read

$$
\begin{gathered}
\boldsymbol{\zeta}^{-}-\boldsymbol{\zeta}^{+}=\gamma_{\mathbf{t}}^{+} \mathbf{e}_{i} \quad \text { in } \boldsymbol{H}_{\times}^{-\frac{1}{2}}\left(\operatorname{div}_{\Gamma}, \Gamma_{o}\right), \\
\boldsymbol{\lambda}^{-}-\boldsymbol{\lambda}^{+}=\gamma_{\mathbf{t}}^{+} \mathbf{h}_{i} \quad \text { in } \boldsymbol{H}_{\times, 00}^{-\frac{1}{2}}\left(\operatorname{div}_{\Gamma}, \Gamma_{o}\right) .
\end{gathered}
$$

These transmission conditions are due to the fact that $\mathbf{e}$ denotes the total field in $\Omega_{s}$, whereas in $\Omega^{\prime}$ it refers only to the scattered field that satisfies the Silver-Müller radiation condition at $\infty$.

For the sake of completeness we note that

$$
\boldsymbol{\zeta}^{-}=0 \quad, \quad \boldsymbol{\zeta}^{+}=-\gamma_{\mathbf{t}}^{+} \mathbf{e}_{i} \quad \text { on } \Gamma_{\mathrm{PEC}} .
$$

Let us rewrite (47) as

$$
\begin{aligned}
& \left(\begin{array}{cc}
-\frac{1}{2} \mathrm{Id}+\mathbf{C}_{\kappa^{-}} & \frac{\mu^{-}}{\kappa^{-}} \mathbf{S}_{\kappa^{-}} \\
\frac{\kappa^{-}}{\mu^{-}} \mathbf{S}_{\kappa^{-}} & -\frac{1}{2} \mathrm{Id}+\mathbf{C}_{\kappa^{-}}
\end{array}\right)\left(\begin{array}{c}
\boldsymbol{\zeta}^{-} \\
\boldsymbol{\lambda}^{-}
\end{array}\right)=0, \\
& \left(\begin{array}{cc}
-\frac{1}{2} \mathrm{Id}-\mathbf{C}_{\kappa^{+}} & -\frac{\mu_{0}}{\kappa^{+}} \mathbf{S}_{\kappa^{+}} \\
-\frac{\kappa^{+}}{\mu_{0}} \mathbf{S}_{\kappa^{+}} & -\frac{1}{2} \mathrm{Id}-\mathbf{C}_{\kappa^{+}}
\end{array}\right)\left(\begin{array}{c}
\boldsymbol{\zeta}^{+} \\
\boldsymbol{\lambda}^{+}
\end{array}\right)=0 .
\end{aligned}
$$

From these equations we aim to derive the crucial electric to magnetic maps, which provide the Poincaré-Stekhlov operators for electromagnetic scattering. First, we use the second equations in (77) and (78) and get

$$
\begin{aligned}
& \boldsymbol{\lambda}^{-}=\left(\frac{\kappa^{-}}{\mu^{-}} \mathbf{S}_{\kappa^{-}}\right) \boldsymbol{\zeta}^{-}+\left(\frac{1}{2} \mathrm{Id}+\mathbf{C}_{\kappa^{-}}\right) \boldsymbol{\lambda}^{-}, \\
& \boldsymbol{\lambda}^{+}=\left(-\frac{\kappa^{+}}{\mu_{0}} \mathbf{S}_{\kappa^{+}}\right) \boldsymbol{\zeta}^{+}+\left(\frac{1}{2} \mathrm{Id}-\mathbf{C}_{\kappa^{+}}\right) \boldsymbol{\lambda}^{+} .
\end{aligned}
$$

Then, we rely on the first equations to eliminate the magnetic traces remaining on the right hand side: 


$$
\begin{aligned}
& \boldsymbol{\lambda}^{-}=\left(\frac{\kappa^{-}}{\mu^{-}} \mathbf{S}_{\kappa^{-}}-\left(\frac{1}{2} \operatorname{Id}+\mathbf{C}_{\kappa^{-}}\right)\left(\frac{\mu^{-}}{\kappa^{-}} \mathbf{S}_{\kappa^{-}}\right)^{-1}\left(-\frac{1}{2} \operatorname{Id}+\mathbf{C}_{\kappa^{-}}\right)\right) \boldsymbol{\zeta}^{-} \\
& \boldsymbol{\lambda}^{+}=\left(-\frac{\kappa^{+}}{\mu_{0}} \mathbf{S}_{\kappa^{+}}+\left(\frac{1}{2} \operatorname{Id}-\mathbf{C}_{\kappa^{+}}\right)\left(\frac{\mu_{0}}{\kappa^{+}} \mathbf{S}_{\kappa^{+}}\right)^{-1}\left(-\frac{1}{2} \operatorname{Id}-\mathbf{C}_{\kappa^{+}}\right)\right) \boldsymbol{\zeta}^{+}
\end{aligned}
$$

Strictly speaking, this formal manipulation is only valid, if there are no interior resonances, because invertibility of both $\mathbf{S}_{\kappa^{-}}$and $\mathbf{S}_{\kappa^{+}}$has to be guaranteed, see Sect. 7.1. For the sake of brevity let us introduce the operators

$$
\begin{aligned}
& \mathrm{P}^{-}:=\frac{\kappa^{-}}{\mu^{-}} \mathbf{S}_{\kappa^{-}}-\left(\frac{1}{2} \mathrm{Id}+\mathbf{C}_{\kappa^{-}}\right)\left(\frac{\mu^{-}}{\kappa^{-}} \mathbf{S}_{\kappa^{-}}\right)^{-1}\left(-\frac{1}{2} \mathrm{Id}+\mathbf{C}_{\kappa^{-}}\right) \\
& \mathrm{P}^{+}:=-\frac{\kappa^{+}}{\mu_{0}} \mathbf{S}_{\kappa^{+}}+\left(\frac{1}{2} \mathrm{Id}-\mathbf{C}_{\kappa^{+}}\right)\left(\frac{\mu_{0}}{\kappa^{+}} \mathbf{S}_{\kappa^{+}}\right)^{-1}\left(-\frac{1}{2} \operatorname{Id}-\mathbf{C}_{\kappa^{+}}\right) .
\end{aligned}
$$

By Cor. 2 they map continuously

$$
\mathrm{P}^{-}, \mathrm{P}^{+}: \boldsymbol{H}_{\times}^{-\frac{1}{2}}\left(\operatorname{div}_{\Gamma}, \Gamma\right) \mapsto \boldsymbol{H}_{\times}^{-\frac{1}{2}}\left(\operatorname{div}_{\Gamma}, \Gamma\right) .
$$

Moreover, the derivation of $\mathrm{P}^{-}$and $\mathrm{P}^{+}$confirms that barring interior resonances, $\left(\boldsymbol{\zeta}^{-}, \mathrm{P}^{-} \boldsymbol{\zeta}^{-}\right)$and $\left(\boldsymbol{\zeta}^{+}, \mathrm{P}^{+} \boldsymbol{\zeta}^{+}\right)$will be interior/exterior electromagnetic Cauchy data.

Using these operators, the transmission condition (76) can be stated as

$$
\mathrm{P}^{-} \boldsymbol{\zeta}^{-}-\mathrm{P}^{+} \boldsymbol{\zeta}^{+}=\gamma_{\mathbf{t}}^{+} \mathbf{h}_{i} \quad \text { in } \boldsymbol{H}_{\times, 00}^{-\frac{1}{2}}\left(\operatorname{div}_{\Gamma}, \Gamma_{o}\right)
$$

Plugging in (75) and retaining $\zeta:=\zeta^{-}$as unknown, this can be cast into the equivalent variational form

$$
\left\langle\left(\mathrm{P}^{-} \boldsymbol{\zeta}-\mathrm{P}^{+}\left(\boldsymbol{\zeta}-\gamma_{\mathbf{t}}^{+} \mathbf{e}_{i}\right), \boldsymbol{\mu}\right\rangle_{\boldsymbol{\tau}, \Gamma}=\left\langle\gamma_{\mathbf{t}}^{+} \mathbf{h}_{i}, \boldsymbol{\mu}\right\rangle_{\boldsymbol{\tau}, \Gamma} \quad \forall \boldsymbol{\mu} \in \boldsymbol{H}_{\times}^{-\frac{1}{2}}\left(\operatorname{div}_{\Gamma}, \Gamma_{o}\right) .\right.
$$

The presence of inverse operators in the definitions of $\mathrm{P}^{-}$and $\mathrm{P}^{+}$makes (83) unsuitable for a direct Galerkin discretization. The usual trick to avoid these undesirable inverses is to use (79) and (80) and switch to a mixed formulation:

$$
\begin{aligned}
\mathrm{P}^{-} \boldsymbol{\zeta} & =\left(\frac{\kappa^{-}}{\mu^{-}} \mathbf{S}_{\kappa^{-}}\right) \boldsymbol{\zeta}+\left(\frac{1}{2} \mathrm{Id}+\mathbf{C}_{\kappa^{-}}\right) \boldsymbol{\lambda}^{-}, \boldsymbol{\lambda}^{-}:=-\left(\frac{\mu^{-}}{\kappa^{-}} \mathbf{S}_{\kappa^{-}}\right)^{-1}\left(-\frac{1}{2} \operatorname{Id}+\mathbf{C}_{\kappa^{-}}\right) \boldsymbol{\zeta}, \\
\mathrm{P}^{+} \boldsymbol{\zeta}^{+} & =\left(-\frac{\kappa^{+}}{\mu_{0}} \mathbf{S}_{\kappa^{+}}\right) \boldsymbol{\zeta}^{+}+\left(\frac{1}{2} \operatorname{Id}-\mathbf{C}_{\kappa^{+}}\right) \boldsymbol{\lambda}^{+}, \boldsymbol{\lambda}^{+}:=\left(\frac{\mu_{0}}{\kappa^{+}} \mathbf{S}_{\kappa^{+}}\right)^{-1}\left(-\frac{1}{2} \operatorname{Id}-\mathbf{C}_{\kappa^{+}}\right) \boldsymbol{\zeta}^{+},
\end{aligned}
$$

where $\boldsymbol{\lambda}^{-}, \boldsymbol{\lambda}^{+} \in \boldsymbol{H}_{\times}^{-\frac{1}{2}}\left(\operatorname{div}_{\Gamma}, \Gamma\right)$ can be regarded as auxiliary unknowns defined on all of $\Gamma$. Hence, (83) is equivalent to: seek $\boldsymbol{\zeta} \in \boldsymbol{H}_{\times, 00}^{-\frac{1}{2}}\left(\operatorname{div}_{\Gamma}, \Gamma_{o}\right)$, $\lambda^{-} \in \boldsymbol{H}_{\times}^{-\frac{1}{2}}\left(\operatorname{div}_{\Gamma}, \Gamma\right), \lambda^{+} \in \boldsymbol{H}_{\times}^{-\frac{1}{2}}\left(\operatorname{div}_{\Gamma}, \Gamma\right)$ such that

$$
\begin{aligned}
& \begin{aligned}
\left\langle\left(\frac{\kappa^{-}}{\mu^{-}} \mathbf{S}_{\kappa^{-}}\right) \boldsymbol{\zeta}+\left(\frac{\kappa^{+}}{\mu_{0}} \mathbf{S}_{\kappa^{+}}\right)\right. & \left.\left.\left(\boldsymbol{\zeta}-\gamma_{\mathbf{t}}^{+} \mathbf{e}_{i}\right), \boldsymbol{\mu}\right\rangle_{\underset{\boldsymbol{\tau}}{*}, \mathcal{\gamma}^{-}}^{+}, \boldsymbol{\mu}\right\rangle_{\boldsymbol{\tau}, \Gamma}+ \\
& +\left\langle\left(\frac{1}{2} \mathrm{Id}+\mathbf{C}_{\kappa^{-}}+\right.\right.
\end{aligned} \\
& -\left\langle\left(\frac{1}{2} \operatorname{Id}-\mathbf{C}_{\kappa^{+}}\right) \boldsymbol{\lambda}^{+}, \boldsymbol{\mu}\right\rangle_{\boldsymbol{\tau}, \Gamma}=\left\langle\gamma_{\mathbf{t}}^{+} \mathbf{h}_{i}, \boldsymbol{\mu}\right\rangle_{\boldsymbol{\tau}, \Gamma}, \\
& \left\langle\left(-\frac{1}{2} \mathrm{Id}+\mathbf{C}_{\kappa^{-}}\right) \boldsymbol{\zeta}, \boldsymbol{\tau}\right\rangle_{\boldsymbol{\tau}, \Gamma}+\left\langle\left(\frac{\mu^{-}}{\kappa^{-}} \mathbf{S}_{\kappa^{-}}\right) \boldsymbol{\lambda}^{-}, \boldsymbol{\tau}\right\rangle_{\boldsymbol{\tau}, \Gamma}=0, \\
& \left\langle\left(\frac{1}{2} \operatorname{Id}+\mathbf{C}_{\kappa^{+}}\right)\left(\boldsymbol{\zeta}-\gamma_{\mathbf{t}}^{+} \mathbf{e}_{i}\right), \boldsymbol{\theta}\right\rangle_{\boldsymbol{\tau}, \Gamma}+\left\langle\left(\frac{\mu_{0}}{\kappa^{+}} \mathbf{S}_{\kappa^{+}}\right) \boldsymbol{\lambda}^{+}, \boldsymbol{\theta}\right\rangle_{\boldsymbol{\tau}, \Gamma}=0 .
\end{aligned}
$$

for all $\boldsymbol{\mu} \in \boldsymbol{H}_{\times}^{-\frac{1}{2}}\left(\operatorname{div}_{\Gamma}, \Gamma_{o}\right), \boldsymbol{\tau} \in \boldsymbol{H}_{\times}^{-\frac{1}{2}}\left(\operatorname{div}_{\Gamma}, \Gamma\right), \boldsymbol{\theta} \in \boldsymbol{H}_{\times}^{-\frac{1}{2}}\left(\operatorname{div}_{\Gamma}, \Gamma\right)$. 
Lemma 18. The variational problem (84) has a unique solution $\left(\zeta^{-}, \lambda^{+}, \lambda^{-}\right) \in$ $\boldsymbol{H}_{\times}^{-\frac{1}{2}}\left(\operatorname{div}_{\Gamma}, \Gamma_{o}\right) \times \boldsymbol{H}_{\times}^{-\frac{1}{2}}\left(\operatorname{div}_{\Gamma}, \Gamma\right) \times \boldsymbol{H}_{\times}^{-\frac{1}{2}}\left(\operatorname{div}_{\Gamma}, \Gamma\right)$, provided that $\kappa^{+}$does not coincide with an interior electric Maxwell eigenvalue of $\Omega_{s}$.

Proof. We study a solution $\left(\boldsymbol{\zeta}, \boldsymbol{\lambda}^{+}, \boldsymbol{\lambda}^{-}\right)$of the homogeneous system with $\gamma_{\mathbf{t}}^{+} \mathbf{e}_{i}=$ and $\gamma_{\mathbf{t}}^{+} \mathbf{h}_{i}=0$. Then set

$$
\begin{gathered}
\left(\begin{array}{c}
\widetilde{\boldsymbol{\zeta}}^{+} \\
\tilde{\boldsymbol{\lambda}}^{+}
\end{array}\right):=\left(\begin{array}{cc}
\frac{1}{2} \mathrm{Id}-\mathbf{C}_{\kappa^{-}} & -\frac{\mu^{-}}{\kappa^{-}} \mathbf{S}_{\kappa^{-}} \\
-\frac{\kappa^{-}}{\mu^{-}} \widehat{\mathbf{S}}_{\kappa^{-}} & \frac{1}{2} \operatorname{Id}-\mathbf{C}_{\kappa^{-}}
\end{array}\right)\left(\begin{array}{c}
\boldsymbol{\zeta} \\
\boldsymbol{\lambda}^{-}
\end{array}\right), \\
\left(\begin{array}{l}
\widetilde{\boldsymbol{\zeta}}^{-} \\
\tilde{\boldsymbol{\lambda}}^{-}
\end{array}\right):=\left(\begin{array}{cc}
\frac{1}{2} \mathrm{Id}+\mathbf{C}_{\kappa^{+}} & \frac{\mu_{0}}{\kappa^{+}} \mathbf{S}_{\kappa^{+}} \\
\frac{\kappa^{-}}{\mu_{0}} \widehat{\mathbf{S}}_{\kappa^{+}} & \frac{1}{2} \mathrm{Id}+\mathbf{C}_{\kappa^{+}}
\end{array}\right)\left(\begin{array}{c}
\boldsymbol{\zeta} \\
\boldsymbol{\lambda}^{+}
\end{array}\right),
\end{gathered}
$$

Please note that the operators in (85) and (86) are the (scaled) exterior Calderón projector for the interior wave number $\kappa^{-}$and the interior Calderón projector for the exterior wave number $\kappa^{+}$. This means that $\left(\frac{\widetilde{\boldsymbol{\zeta}}^{+}}{\widetilde{\boldsymbol{\lambda}}^{+}}\right)$are exterior Maxwell Cauchy data, whereas $\left(\begin{array}{c}\widetilde{\boldsymbol{\zeta}}^{-} \\ \widetilde{\lambda}^{-}\end{array}\right)$turn out to be interior Maxwell Cauchy data.

From the second and third equation of (84) with zero r.h.s. it is immediate that

$$
\widetilde{\zeta}^{-}=\widetilde{\zeta^{+}}=0
$$

Thus, the unique solvability of the exterior scattering problem yields $\widetilde{\boldsymbol{\lambda}}^{+}=0$. If $\kappa^{+}$ is different from an interior electric Maxwell eigenvalue, then we can also conclude $\lambda^{-}=0$.

Hence, we have shown

$$
\begin{aligned}
& \left(\begin{array}{cc}
\frac{1}{2} \mathrm{Id}+\mathbf{C}_{\kappa^{-}} & \frac{\mu^{-}}{\kappa^{-}} \mathbf{S}_{\kappa^{-}} \\
\frac{\kappa^{-}}{\mu^{-}} \widehat{\mathbf{S}}_{\kappa^{-}} & \frac{1}{2} \mathrm{Id}+\mathbf{C}_{\kappa^{-}}
\end{array}\right)\left(\begin{array}{c}
\boldsymbol{\zeta} \\
\boldsymbol{\lambda}^{-}
\end{array}\right)=\left(\begin{array}{c}
\boldsymbol{\zeta} \\
\boldsymbol{\lambda}^{-}
\end{array}\right), \\
& \left(\begin{array}{cc}
\frac{1}{2} \mathrm{Id}-\mathbf{C}_{\kappa^{+}} & -\frac{\mu_{0}}{\kappa^{+}} \mathbf{S}_{\kappa^{+}} \\
-\frac{\kappa^{-}}{\mu_{0}} \widehat{\mathbf{S}}_{\kappa^{+}} & \frac{1}{2} \mathrm{Id}-\mathbf{C}_{\kappa^{+}}
\end{array}\right)\left(\begin{array}{c}
\boldsymbol{\zeta} \\
\boldsymbol{\lambda}^{+}
\end{array}\right)=\left(\begin{array}{c}
\boldsymbol{\zeta} \\
\boldsymbol{\lambda}^{+}
\end{array}\right) .
\end{aligned}
$$

This means that $\left(\begin{array}{c}\zeta \\ \lambda^{-}\end{array}\right)$are Cauchy data for the the interior scattering problem with wave number $\kappa^{-}$and $\left(\begin{array}{c}\zeta \\ \lambda^{+}\end{array}\right)$play the same role for an exterior scattering problem with wave number $\kappa^{+}$.

Moreover, from the first equation of (84) we can infer that

$$
\begin{aligned}
\boldsymbol{\lambda}^{-}-\boldsymbol{\lambda}^{+}=\left(\frac{\kappa^{-}}{\mu^{-}} \widehat{\mathbf{S}}_{\kappa^{-}}+\right. & \left.\frac{\kappa^{-}}{\mu_{0}} \widehat{\mathbf{S}}_{\kappa^{+}}\right) \boldsymbol{\zeta}+ \\
& +\left(\frac{1}{2} \operatorname{Id}+\mathbf{C}_{\kappa^{-}}\right) \boldsymbol{\lambda}^{-}-\left(\frac{1}{2} \operatorname{Id}-\mathbf{C}_{\kappa^{+}}\right) \boldsymbol{\lambda}^{+}=0 \quad \text { on } \Gamma_{o} .
\end{aligned}
$$

Summing up, the boundary data $\left(\boldsymbol{\zeta}, \boldsymbol{\lambda}^{-}, \boldsymbol{\lambda}^{+}\right)$are the traces of the electric field and the magnetic field, respectively, that solve the scattering problem for the coated dielectric object $\Omega$. Since we considered the case of zero excitation, the unique solvability of the scattering problem enforces $\zeta=\boldsymbol{\lambda}^{-}=\boldsymbol{\lambda}^{+}=0$. 
The bilinear form a associated with the variational problem (84) reads

$$
\begin{aligned}
\underline{\mathrm{a}}\left(\left(\begin{array}{c}
\boldsymbol{\zeta} \\
\boldsymbol{\lambda}^{-} \\
\boldsymbol{\lambda}^{+}
\end{array}\right),\left(\begin{array}{c}
\boldsymbol{\mu} \\
\boldsymbol{\tau} \\
\boldsymbol{\theta}
\end{array}\right)\right):= & \left\langle\left(\frac{\kappa^{-}}{\mu^{-}} \mathbf{S}_{\kappa^{-}}+\frac{\kappa^{+}}{\mu_{0}} \mathbf{S}_{\kappa^{+}}\right) \boldsymbol{\zeta}, \boldsymbol{\mu}\right\rangle_{\boldsymbol{\tau}, \Gamma}- \\
& -\left\langle\left(\frac{1}{2} \mathrm{Id}+\mathbf{C}_{\kappa^{-}}\right) \boldsymbol{\lambda}^{-}, \boldsymbol{\mu}\right\rangle_{\boldsymbol{\tau}, \Gamma}+ \\
& +\left\langle\left(\frac{1}{2} \mathrm{Id}-\mathbf{C}_{\kappa^{+}}\right) \boldsymbol{\lambda}^{+}, \boldsymbol{\mu}\right\rangle_{\boldsymbol{\tau}, \Gamma}+ \\
& +\left\langle\left(-\frac{1}{2} \mathrm{Id}+\mathbf{C}_{\kappa^{-}}\right) \boldsymbol{\zeta}, \boldsymbol{\tau}\right\rangle_{\boldsymbol{\tau}, \Gamma}+ \\
& +\left\langle\left(\frac{1}{2} \operatorname{Id}+\mathbf{C}_{\kappa^{+}}\right) \boldsymbol{\zeta}, \boldsymbol{\theta}\right\rangle_{\boldsymbol{\tau}, \Gamma}+ \\
& +\left\langle\left(\frac{\mu^{-}}{\kappa^{-}} \mathbf{S}_{\kappa^{-}}\right) \boldsymbol{\lambda}^{-}, \boldsymbol{\tau}\right\rangle_{\boldsymbol{\tau}, \Gamma}+ \\
& +\left\langle\left(\frac{\mu_{0}}{\kappa^{+}} \mathbf{S}_{\kappa^{+}}\right) \boldsymbol{\lambda}^{+}, \boldsymbol{\theta}\right\rangle_{\boldsymbol{\tau}, \Gamma},
\end{aligned}
$$

Lemma 19. The bilinear form $\underline{a}$ is satisfies a generalized Gårding inequality on $\left(\boldsymbol{H}_{\times}^{-\frac{1}{2}}\left(\operatorname{div}_{\Gamma}, \Gamma\right)\right)^{3}$.

Proof.

Wrong sign somewhere 